TRANSACTIONS OF THE

AMERICAN MATHEMATICAL SOCIETY

Volume 356, Number 2, Pages 621-658

S 0002-9947(03)03179-9

Article electronically published on September 22, 2003

\title{
EXAMPLES OF PLEATING VARIETIES FOR TWICE PUNCTURED TORI
}

\author{
RAQUEL DÍAZ AND CAROLINE SERIES
}

\begin{abstract}
We give an explicit description of some pleating varieties (sets with a fixed set of bending lines in the convex hull boundary) in the quasiFuchsian space of the twice punctured torus. In accordance with a conjecture of the second author, we show that their closures intersect Fuchsian space in the simplices of minima introduced by Kerckhoff. All computations are done using complex Fenchel-Nielsen coordinates for quasi-Fuchsian space referred to a maximal system of curves.
\end{abstract}

\section{INTRODUCTION}

In a series of papers [9, [10], [11], [12] (see also [19]), the second author and L. Keen have developed the theory of pleating coordinates for quasi-Fuchsian space for the once-punctured torus: to each representation $\rho$ in quasi-Fuchsian space $\mathcal{Q F}$, but not in Fuchsian space $\mathcal{F}$, are assigned two projective measured laminations $\left[\mathrm{pl}^{+}\right],\left[\mathrm{pl}^{-}\right]$and two positive real numbers. The laminations $\left[\mathrm{pl}^{+}\right],\left[\mathrm{pl}^{-}\right]$are the projective classes of the bending measures of the two components of the boundary of the convex hull of the hyperbolic manifold represented by $\rho$. The real numbers in question are the lengths of the laminations $j\left(\left[\mathrm{pl}^{+}\right]\right)$and $j\left(\left[\mathrm{pl}^{-}\right]\right)$, where $j$ is a fixed continuous section from $\mathrm{P} \mathcal{M L}$ to $\mathcal{M L}$, the spaces of projective measured laminations and measured laminations respectively. This gives an embedding from $\mathcal{Q F}-\mathcal{F}$ into $\mathrm{P} \mathcal{M} \mathcal{L}^{2} \times \mathbb{R}^{2}$, and the main object of [12] is to give a complete description of its image. Given two projective measured laminations $[\mu],[\nu]$, the pleating plane $\mathcal{P}_{[\mu],[\nu]}$ is defined to be the subset of $\mathcal{Q F}$ so that $\left[\mathrm{pl}^{+}\right]=[\mu]$ and $\left[\mathrm{pl}^{-}\right]=[\nu]$. The space $\mathcal{Q F}-\mathcal{F}$ is foliated by pleating planes, and it is shown in [12] that each pleating plane is homeomorphic to a connected open set in $\mathbb{R}^{2}$ whose closure intersects $\mathcal{F}$ in the Kerckhoff line of minima determined by $[\mu]$ and $[\nu]$. The remaining boundary of $\mathcal{P}_{[\mu],[\nu]}$ in the representation space $\mathcal{R}$ is formed by two half-lines (meeting at a single point) consisting of cusp groups for which one or the other of the two laminations $\mu, \nu$ has zero length. These considerations allow an explicit computation of the image of the embedding of $\mathcal{Q F}$ into $\mathbb{C}^{2}$ for any given holomorphic coordinates for $\mathcal{R}$.

It is natural to think about generalizing this theory to quasi-Fuchsian space for surfaces of higher genus or with more punctures. One would expect $\mathcal{Q F}-\mathcal{F}$ to be foliated by two-dimensional pleating planes. On trying to carry out this programme, however, some new difficulties appear. Despite the terminology, it is

Received by the editors August 21, 2001 and, in revised form, July 11, 2002.

2000 Mathematics Subject Classification. Primary 30F40, 20H10, 32G15.

(C)2003 American Mathematical Society 
a priori not at all clear that the 'pleating plane' $\mathcal{P}_{[\mu],[\nu]}$ is either connected or a submanifold, nor where it meets $\mathcal{F}$. It should therefore be instructive to look at some concrete examples. The simpler cases are the rational pleating planes, that is, those whose bending measures are supported on a union of closed geodesics. It turns out to be easier to study pleating varieties rather than pleating planes: given $\mathcal{A}=\left\{\alpha_{1}, \ldots, \alpha_{n}\right\}$ and $\mathcal{B}=\left\{\beta_{1}, \ldots, \beta_{m}\right\}$ two collections of disjoint, simple, closed curves on a surface $S$, the pleating variety associated to $\mathcal{A}, \mathcal{B}$ is the subset

$$
\mathcal{P}_{\mathcal{A}, \mathcal{B}}=\left\{\rho \in \mathcal{Q} \mathcal{F}(S)|| \mathrm{pl}^{+}\left|=\alpha_{1} \cup \cdots \cup \alpha_{n},\right| \mathrm{pl}^{-} \mid=\beta_{1} \cup \cdots \cup \beta_{m}\right\} .
$$

(Here $\left|\mathrm{pl}^{ \pm}\right|$denotes the support of the lamination $\mathrm{pl}^{ \pm}$.) In [20] it is conjectured that $\mathcal{P}_{\mathcal{A}, \mathcal{B}}$ meets $\mathcal{F}$ in the simplex of minima $\mathcal{S}_{\mathcal{A}, \mathcal{B}}$ consisting of the lines of minima for all pairs of laminations with support $\mathcal{A}$ and $\mathcal{B}$ respectively, and partial results are proved. Thus these simplices of minima (see Section 4) also become a natural object of study.

In this paper we focus on quasi-Fuchsian space for the twice punctured torus, $\mathcal{Q} \mathcal{F}\left(S_{1,2}\right)$, and study two specific examples. Consider $\mathcal{A}=\left\{\alpha_{1}, \alpha_{2}\right\}, \mathcal{B}=\left\{\beta_{1}, \beta_{2}\right\}$, and $\mathcal{D}=\left\{\beta_{1}, \delta_{1}\right\}$, where $\alpha_{1}, \alpha_{2}$ is a pair of non-separating, disjoint, simple closed curves on $S=S_{1,2} ; \beta_{1}, \beta_{2}$ is another pair of the same kind such that each $\beta_{i}$ intersects each $\alpha_{j}$ exactly once; and finally, $\delta_{1}$ is a separating curve, disjoint from $\beta_{1}$ and $\alpha_{2}$ and intersecting $\alpha_{1}$ twice. Using complex Fenchel-Nielsen coordinates, we exactly locate the simplices of minima $\mathcal{S}_{\mathcal{A}, \mathcal{B}}$ and $\mathcal{S}_{\mathcal{A}, \mathcal{D}}$, and the pleating varieties $\mathcal{P}_{\mathcal{A}, \mathcal{B}}$ and $\mathcal{P}_{\mathcal{A}, \mathcal{D}}$. We show that the intersection of the closure of these pleating varieties with $\mathcal{F}$ is the closure of the corresponding simplices of minima. These two examples serve to illustrate a striking distinction between simplices: $\mathcal{S}_{\mathcal{A}, \mathcal{B}}$ is non-degenerate, in the sense that it is a 3 -dimensional submanifold of the 4-dimensional space $\mathcal{F}$, while $\mathcal{S}_{\mathcal{A}, \mathcal{D}}$ is 2 -dimensional. Nevertheless, as expected, both pleating varieties have (real) dimension 4. Our study leads us naturally to find representations for cusp groups in the boundary of $\mathcal{P}_{\mathcal{A}, \mathcal{B}}$ and $\mathcal{P}_{\mathcal{A}, \mathcal{D}}$. We also locate some of the adjacent pleating varieties, and the pleating plane in the interior of $\mathcal{P}_{\mathcal{A}, \mathcal{B}}$ on which the two bending angles on $\alpha_{1}$ and $\alpha_{2}$ are equal.

Following [20], we know that in general the main necessary condition for a point $\rho$ to be in a simplex of minima $\mathcal{S}_{\mathcal{A}, \mathcal{B}}$ is that the determinant of a certain matrix of derivatives of length functions vanishes, see Proposition 4.5. In the special cases under consideration, we are able to work out explicitly where this occurs. Two necessary conditions must be imposed to obtain the pleating variety $\mathcal{P}_{\mathcal{A}, \mathcal{B}}$ :

(1) If $\rho \in \mathcal{Q F}$, and if a closed geodesic $\gamma$ is contained in the support of $\mathrm{pl}^{ \pm}$, then the complex length $\lambda_{\gamma}(\rho)$ must be real ([20], Corollary 3.4). Hence, $\operatorname{tr} \rho(\gamma)$ must be real, for all $\gamma$ in $\mathcal{A}$ and $\mathcal{B}$.

(2) Since the convex hull boundary bounds a convex set in $\mathbb{H}^{3}$, if the bending measure $\mathrm{pl}^{+}$is supported on the union of several closed geodesics $\left\{\alpha_{i}\right\}$, then the bending angles on all of these $\alpha_{i}$ must have the same sign.

Conditions (1) and (2) are not in general sufficient to ensure that $\rho \in \mathcal{P}_{\mathcal{A}, \mathcal{B}}$. However, they are sufficient if the point $\rho$ is connected with $\mathcal{F}$ by a path along which (1) and (2) hold. This is the main fact we use.

The paper is organized as follows. Section 2 contains background on Teichmüller and quasi-Fuchsian spaces and complex Fenchel-Nielsen coordinates. In Section 3 we derive explicit formulas for a representation of $\pi_{1}\left(S_{1,2}\right)$ into $\operatorname{PSL}(2, \mathbb{C})$ in terms of Fenchel-Nielsen coordinates with respect to the pants decomposition $\left\{\alpha_{1}, \alpha_{2}\right\}$ 
and dual curves $\left\{\delta_{1}, \delta_{2}\right\}$, together with various other formulae for traces which are useful later. In Section 4 we compute the simplices of minima $\mathcal{S}_{\mathcal{A}, \mathcal{B}}$ and $\mathcal{S}_{\mathcal{A}, \mathcal{D}}$, and finally in Section 5 we compute the pleating varieties $\mathcal{P}_{\mathcal{A}, \mathcal{B}}$ and $\mathcal{P}_{\mathcal{A}, \mathcal{D}}$.

Our results depend heavily on computations. We use the hyperboloid model of hyperbolic space to compute distances and angles in the hyperbolic plane. (Although we study three-dimensional hyperbolic structures, most computations are done in some hyperbolic plane inside hyperbolic 3-space.) We review all the formulae needed in Section 2.1. The main computation is the one given in Proposition 3.2 in which we separate the real and imaginary parts of the traces of certain isometries of $\mathbb{H}^{3}$. The expression obtained is written in terms of $\left\langle V_{i}, e_{j}\right\rangle$, where $\langle\cdot, \cdot\rangle$ is the Minkowski inner product and the $V_{i}$ and $e_{j}$ refer to certain points and lines shown in what we call the Main Figure (Figure 4). We will exploit the geometric meaning of the Main Figure and use it repeatedly in our arguments throughout the paper.

At the time we were writing this paper we learned that Dragomir Šarić had independently partially worked out some similar examples; this work is unpublished.

\section{BACKGROUND}

2.1. Hyperbolic geometry and Kleinian groups. To make computations in the hyperbolic plane we use the hyperboloid model. We refer to 24] for a detailed description, and describe here only what we need. We consider the vector space $\mathbb{R}^{3}$ with the bilinear form $\langle v, w\rangle=v_{1} w_{1}+v_{2} w_{2}-v_{3} w_{3}$. The hyperbolic plane, $\mathbb{H}^{2}$, is the subset of vectors $v$ with $\langle v, v\rangle=-1$ and $v_{3}>0$. Hyperbolic lines (i.e., geodesics) are given by the intersection of $\mathbb{H}^{2}$ with 2-dimensional linear subspaces of $\mathbb{R}^{3}$. A hyperbolic line, $H$, determines two closed halfplanes, $H^{+}, H^{-}$. Given a halfplane $H^{-}$, there is unique vector $e$ with $\langle e, e\rangle=1$ and $\langle e, v\rangle \leq 0$ for all $v \in H^{-}$. The vector $e$ is called the outward unit normal to $H^{-}$. The inner product of two vectors which are either points of $\mathbb{H}^{2}$ or unitary vectors has a geometric meaning in terms of distances or angles. If $V_{1}, V_{2} \in \mathbb{H}^{2}$, then $\left\langle V_{1}, V_{2}\right\rangle=-\cosh \mathrm{d}\left(V_{1}, V_{2}\right)$, where $\mathrm{d}\left(V_{1}, V_{2}\right)$ is the hyperbolic distance between $V_{1}$ and $V_{2}$. If $H^{-}$is a halfplane and $e$ its outward unit normal, then $\left\langle V_{1}, e\right\rangle= \pm \sinh \mathrm{d}\left(V_{1}, H\right)$, where $\mathrm{d}\left(V_{1}, H\right)$ is the hyperbolic distance between the point $V_{1}$ and the boundary $H$ of $H^{-}$, and the sign is taken negative if the point $V_{1}$ is contained in the halfplane $H^{-}$, and positive otherwise. Notice that $\left\langle V_{1}, e\right\rangle=0$ when $V_{1}$ is on the line $H$. If $H_{1}^{-}, H_{2}^{-}$ are halfspaces with outward unit vectors $e_{1}, e_{2}$, then $\left\langle e_{1}, e_{2}\right\rangle=-\cos \theta$ if $H_{1}, H_{2}$ intersect, where $\theta$ is the angle of intersection of $H_{1}^{-}, H_{2}^{-}$. Otherwise, $\left\langle e_{1}, e_{2}\right\rangle=$ $\pm \cosh \mathrm{d}\left(H_{1}, H_{2}\right)$, where $\mathrm{d}\left(H_{1}, H_{2}\right)$ is the hyperbolic distance between $H_{1}, H_{2}$ and the sign is determined by the relative position of $H_{1}^{-}$and $H_{2}^{-}$: it is positive when one of the halfplanes contains the other, and negative otherwise.

We consider hyperbolic space $\mathbb{H}^{3}$ with a given (standard) orientation. Given an oriented line $L$ in $\mathbb{H}^{3}$, this orientation determines an orientation in each orthogonal plane; a loxodromic element of Isom $\mathbb{H}^{3}$ with axis $L$ has a well defined complex translation length, $\lambda=l+i \phi \in \mathbb{C} / 2 \pi i$. In this paper we make the convention that $l$ is positive if the translation is in the direction of $L$, and negative otherwise. If $L, L^{\prime}$ are two oriented lines in $\mathbb{H}^{3}$, and $M$ their common perpendicular, with an orientation, then the signed complex distance $\mathbf{d}_{M}\left(L, L^{\prime}\right)$ between $L$ and $L^{\prime}$ along $M$ is the complex translation length of the unique loxodromic element with axis $M$ that takes $L$ into $L^{\prime}$. We note that the real part of the complex distance is the real 
signed distance between $L$ and $L^{\prime}$, while the imaginary part is the angle from $L$ to $L^{\prime}$.

To work with isometries of hyperbolic space it is more convenient to use the Poincaré halfspace model, where we have the useful description

$$
\text { Isom }^{+} \mathbb{H}^{3}=P S L(2, \mathbb{C}) .
$$

If $\varphi \in P S L(2, \mathbb{C})$ is a loxodromic element with complex translation length $\lambda$, represented by a matrix $A \in S L(2, \mathbb{C})$, then we have the relation $\pm \operatorname{tr} A=2 \cosh \frac{\lambda}{2}$. A loxodromic element is purely hyperbolic if $\lambda \in \mathbb{R}$.

A Kleinian group, $\Gamma$, is a discrete subgroup of $P S L(2, \mathbb{C})$. It acts both on hyperbolic space $\mathbb{H}^{3}$ by orientation-preserving isometries, and on its boundary $\hat{\mathbb{C}}=$ $\mathbb{C} \cup \infty$ by Möbius transformations. The domain of discontinuity of $\Gamma$ is the maximal open subset of $\hat{\mathbb{C}}$ on which $\Gamma$ acts properly discontinuously, and the limit set is its complement. The convex hull $\mathcal{C}$ of $\Gamma$ is the convex hull in $\mathbb{H}^{3}$ of its limit set. Its boundary, $\partial \mathcal{C}$, is a (union of) pleated surface $(s)$ invariant by $\Gamma$, so that $\partial \mathcal{C} / \Gamma$ is a (union of) hyperbolic surface(s). Each component of $\partial \mathcal{C}$ is bent along bending lines, which project to a geodesic lamination on the corresponding component of $\partial \mathcal{C} / \Gamma$. This lamination carries a transverse measure, the bending measure. We refer to [4] and [2] for general background about pleated surfaces.

2.2. Teichmüller space and quasi-Fuchsian space. Let $S=S_{g, b}$ be an oriented surface of genus $g$ and $b$ punctures. Let $\rho: \pi_{1}(S) \rightarrow P S L(2, \mathbb{R})$ be a representation which is discrete, faithful and which maps loops around punctures to parabolics. Denote by $\Gamma$ the image of $\rho$. Then $\mathbb{H}^{2} / \Gamma$ is a marked complete hyperbolic surface with finite area. The Teichmüller space of $S$, Teich $(S)$, is the set of such representations modulo conjugation by elements of $\operatorname{PSL}(2, \mathbb{R})$. It is well known that $\operatorname{Teich}\left(S_{g, b}\right)$ is topologically a ball of dimension $6 g-6+2 b$.

More generally, we can consider representations of $\pi_{1}(S)$ into $P S L(2, \mathbb{C})$. The representation variety $\mathcal{R}(S)$ is the set of such representations up to conjugation by elements of $P S L(2, \mathbb{C})$. We shall be interested in the quasi-Fuchsian space of $S$, denoted $\mathcal{Q F}(S)$, which is the subset of $\mathcal{R}(S)$ consisting of the classes of discrete and faithful representations whose image is a quasi-Fuchsian group. A Kleinian group is quasi-Fuchsian if its limit set is a Jordan curve and none of its elements interchanges the two components of the domain of discontinuity. In the particular case in which the limit set is a round circle, the group is called Fuchsian, and the space of classes of Fuchsian groups is called Fuchsian space, denoted $\mathcal{F}(S)$. It is a copy of Teichmüller space inside $\mathcal{Q F}(S)$. By the Bers simultaneous uniformization theorem, $\mathcal{Q F}\left(S_{g, n}\right)$ is homeomorphic to $\operatorname{Teich}\left(S_{g, n}\right) \times \overline{\operatorname{Teich}\left(S_{g, n}\right)}$, so that it is topologically a ball of dimension $2(6 g-6+2 b)$.

If $\Gamma$ is quasi-Fuchsian, not Fuchsian, then the boundary of its convex hull has two components $\partial \mathcal{C}^{+}, \partial \mathcal{C}^{-}$. We denote by $\mathrm{pl}^{+}, \mathrm{pl}^{-}$their bending measures. In this paper we shall only deal with the case in which $\mathrm{pl}^{ \pm}$projects onto a rational lamination on $\partial \mathcal{C}^{ \pm} / \Gamma$ (i.e., its support is a union of closed geodesics). In this case the pleated surface $\partial \mathcal{C}^{ \pm}$is also called rational, and can be regarded as the union of pieces of hyperbolic planes in $\mathbb{H}^{3}$ glued together along geodesics. There can be other rational pleated surfaces invariant by $\rho$. Let $\mathcal{A}=\left\{\alpha_{1}, \ldots, \alpha_{n}\right\}$ be a pants decomposition of $S$, i.e., the curves $\alpha_{i}$ decompose $S$ into pairs of pants; if $\rho \alpha_{i}$ are purely hyperbolic isometries for all $i$, then each pair of pants is covered by flat pieces and all these flat pieces are glued along the axes of $\rho \alpha_{i}$ and their images 
under the group. In this way we obtain a rational pleated surface invariant by $\rho$, which we denote by $\mathrm{Pl}_{\mathcal{A}}$, and we say that $\rho$ realizes the pleated surface $\mathrm{Pl}_{\mathcal{A}}$. The bending lines project onto the geodesics representing loops in $\mathcal{A}$, and we denote by $\theta_{\alpha_{i}}$ the bending angle on the bending line projecting onto $\alpha_{i}$. This signed angle is defined relative to a fixed orientation of $S$, which gives an orientation of $\mathrm{Pl}_{\mathcal{A}}$, as the angle between the outward normal vectors to the two flat pieces, $F_{1}, F_{2}$, meeting at the bending line, with the convention that the angle is positive when the plane containing $F_{1}$ separates its outward normal vector from $F_{2}$. We notice that if $\theta_{\alpha_{i}}=0$ for all $i$, then $\Gamma$ is Fuchsian. Usually, the actual sign of the bending angle is not important, but the relative signs along different bending lines are. The pleated surface $\mathrm{Pl}_{\mathcal{A}}$ can be convex or not, and can be embedded or not. If it is convex and embedded, then it must be a component of $\partial \mathcal{C}$; see [11. We refer to 2 . for more details about pleated surfaces, and to [11] and [20] for their relation with Fenchel-Nielsen coordinates.

2.3. Fenchel-Nielsen coordinates. Fenchel-Nielsen coordinates are the basis for our computations. Let $S=S_{g, b}$ be an oriented surface and $\left\{\alpha_{1}, \ldots, \alpha_{n}\right\}$ a pants decomposition of $S$ (so that $n=3 g-3+2 b$ and the number of pants in the decomposition is $2 g-2+b$ ). We can parametrize Teich $(S)$ by the so-called FenchelNielsen coordinates, $\left(l_{\alpha_{i}}, t_{\alpha_{i}}\right)$, where $l_{\alpha_{i}}$ are the lengths of the curves $\alpha_{i}$ and $t_{\alpha_{i}}$ are the twist parameters, which, intuitively, determine how the two pairs of pants meeting along the curve $\alpha_{i}$ are glued.

In order to define the twist parameters precisely, we need to specify, for each set of fixed values of the lengths of $\alpha_{i}$, a base point $\rho_{0}$ in Teichmüller space, at which all the twist parameters are equal to zero. Once we have this point, given a point $\rho \in \operatorname{Teich}(S)$ with $l_{\alpha_{i}}$ the given values, we can recover $\rho_{0}$ from $\rho$ by doing twists about $\alpha_{i}$ by certain amounts $t_{i}$. Then the twist parameters for $\rho$ are defined to be $t_{\alpha_{i}}=-t_{i}$. To choose the base point, we take a system of curves $\left\{\delta_{1}, \ldots, \delta_{n}\right\}$ dual to the $\left\{\alpha_{1}, \ldots, \alpha_{n}\right\}$, in the sense that the geometric intersection number of $\delta_{i}$ with $\alpha_{j}$ is either 1 or 2 if $i=j$, and 0 if $i \neq j$. (Notice that the dual curves are not necessarily mutually disjoint.) For fixed values of $l_{\alpha_{i}}$, the point $\rho_{0}$ is chosen so that if $\delta_{i}$ and $\alpha_{i}$ intersect once, then the intersection angle is $\pi / 2$, whereas if they intersect twice, the intersection angles are $\theta$ and $\pi-\theta$. We notice that at $\rho_{0}$, if $P, P^{\prime}$ are the two pairs of pants meeting at $\alpha_{i}$, then both pants are glued up so that the common perpendicular arcs in $P$ from $\alpha_{i}$ to the other boundary components match with the common perpendicular arcs in $P^{\prime}$ from $\alpha_{i}$ to the other boundary components.

Kourouniotis [17] and Tan 22] have extended this construction to complex Fenchel-Nielsen coordinates for quasi-Fuchsian space, so that both the lengths and the twists become complex functions $\left(\lambda_{\alpha_{i}}, \tau_{\alpha_{i}}\right)$. Now, from a point in $\mathcal{Q F}$ we can recover the base point (chosen as before) by doing a complex twist about $\alpha_{i}$, where the real part of the twist means a shift and the imaginary part means a rotational angle. This process will be illustrated in more detail in the proof of Theorem 3.1.

There is a certain subtlety involved in defining the length parameters for a point $\rho \in \mathcal{Q F}$, which necessitates using the half lengths $\lambda_{\alpha_{i}} / 2$ rather than the lengths $\lambda_{\alpha_{i}}$. Nevertheless, for the purpose of this paper, we only need those representations $\rho$ for which $\lambda_{\alpha_{i}}=l_{\alpha_{i}} \in \mathbb{R}$ for all $i$. We state the theorem in its generality, but in practice we will always be restricted to this case and will take $l_{\alpha_{i}}$ as our parameter rather than $l_{\alpha_{i}} / 2$. 
Theorem 2.1. (a) (Real Fenchel-Nielsen coordinates.) The map

$$
\Psi: \operatorname{Teich}(S) \rightarrow\left(\mathbb{R}^{+} \times \mathbb{R}\right)^{n}, \rho \mapsto\left(l_{\alpha_{i}}(\rho), t_{\alpha_{i}}(\rho)\right),
$$

is a homeomorphism.

(b) (Complex Fenchel-Nielsen coordinates.) The map

$$
\Psi: \mathcal{Q F}(S) \rightarrow\left(\mathbb{C}^{+} \times \mathbb{C} / 2 \pi i\right)^{n}, \rho \mapsto\left(\frac{\lambda_{\alpha_{i}}}{2}(\rho), \tau_{\alpha_{i}}(\rho)\right),
$$

is an embedding.

Remark. If we choose a different system of dual curves, then we get a new coordinate system $\left(\lambda_{\alpha_{i}}^{\prime} / 2, \tau_{\alpha_{i}}^{\prime}\right)$ which differs from the first coordinate system by a translation of the twist parameters: $\tau_{\alpha_{i}}^{\prime}-\tau_{\alpha_{i}}=N \lambda_{\alpha_{i}}$, with $N \in \mathbb{Z}$.

\section{REPRESENTATION}

We are going to derive an explicit parametrization for $\mathcal{Q F}\left(S_{1,2}\right)$ using FenchelNielsen coordinates with respect to the pants decomposition $\left\{\alpha_{1}, \alpha_{2}\right\}$ and the dual curves $\left\{\delta_{1}, \delta_{2}\right\}$; see Figure 1 . Precisely, this means that given $\left(l_{\alpha_{i}}, \tau_{\alpha_{i}}\right)$ in the image of the map $\Psi$ of Theorem 2.1 we shall find its preimage representation (up to conjugation). In particular, if $\tau_{i}=t_{i} \in \mathbb{R}$, this gives a parametrization of $\mathcal{F}\left(S_{1,2}\right)$.

Fix a presentation $\pi_{1}(S)=\left\langle\alpha_{1}, \alpha_{2}, \beta_{1}\right.$ : -$\rangle$ for the fundamental group of $S$, where $\alpha_{1}, \alpha_{2}, \beta_{1}$ are the oriented loops based on the point $*$ in Figure 1. For later purposes we record expressions for other loops on the surface:

$$
\beta_{2}=\alpha_{1}^{-1} \beta_{1} \alpha_{2}, \quad \delta_{1}=\beta_{1} \alpha_{2} \beta_{1}^{-1} \alpha_{2}^{-1}, \quad \delta_{2}=\alpha_{1} \beta_{1}^{-1} \alpha_{1}^{-1} \beta_{1},
$$

and note that $\alpha_{1} \alpha_{2}^{-1}$ and $\beta_{2} \beta_{1}^{-1}=\alpha_{1}^{-1} \beta_{1} \alpha_{2} \beta_{1}^{-1}$ are the loops around the punctures of $S_{1,2}$. To abbreviate, we shall throughout use the notation $l_{1}=l_{\alpha_{1}}, l_{2}=$ $l_{\alpha_{2}}, \tau_{1}=\tau_{\alpha_{1}}, \tau_{2}=\tau_{\alpha_{2}}$ for the Fenchel-Nielsen coordinates with respect to $\left\{\alpha_{1}, \alpha_{2}\right\}$ and $\left\{\delta_{1}, \delta_{2}\right\}$.

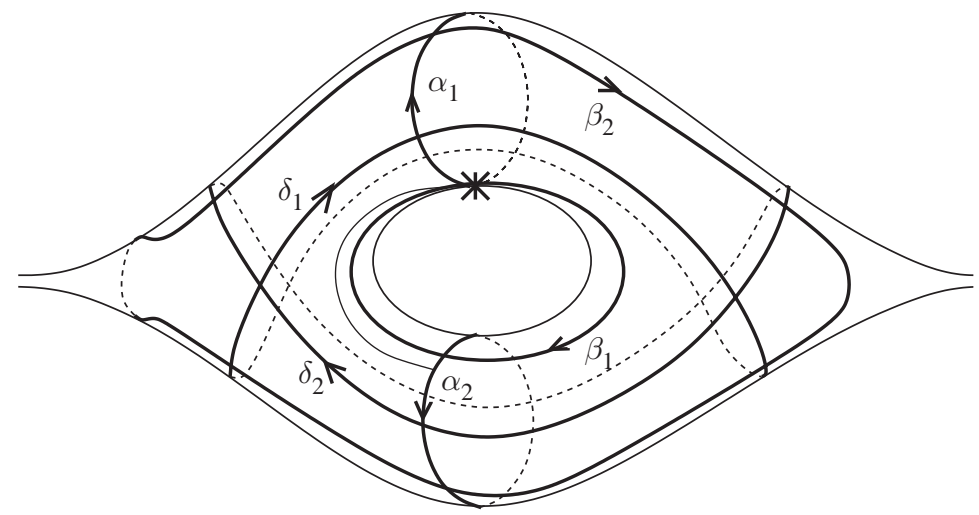

FiguRE 1. The pants decomposition $\left\{\alpha_{1}, \alpha_{2}\right\}$, dual curves $\left\{\delta_{1}, \delta_{2}\right\}$ and the presentation of $\pi_{1}(S)$.

Notation. For a complex number $a$, we define

$$
T_{L_{1}}(a)=\left(\begin{array}{cc}
e^{a / 2} & 0 \\
0 & e^{-a / 2}
\end{array}\right), \quad T_{M}(a)=\left(\begin{array}{cc}
\cosh \frac{a}{2} & \sinh \frac{a}{2} \\
\sinh \frac{a}{2} & \cosh \frac{a}{2}
\end{array}\right) .
$$


For an oriented line $N$ in hyperbolic space and a complex number $a$, we denote by $\bar{T}_{N}(a)$ the loxodromic isometry with axis $N$ and complex translation length $a$. Let $p$ be the projection from $S L(2, \mathbb{C})$ to $P S L(2, \mathbb{C})$. Then $p\left(T_{L_{1}}(a)\right)=\bar{T}_{L_{1}}(a)$, where $L_{1}$ is the oriented line, in the upper halfspace model for hyperbolic space, going through $(0,0) \in \mathbb{C} \times \mathbb{R}^{+}$and $(0,1) \in \mathbb{C} \times \mathbb{R}^{+}$. Similarly, $p\left(T_{M}(a)\right)=\bar{T}_{M}(a)$, where $M$ is the oriented line going from $(-1,0) \in \mathbb{C} \times \mathbb{R}+$ to $(1,0)$. If $a$ is real, then we can regard both $\bar{T}_{L_{1}}(a)$ and $\bar{T}_{M}(a)$ either as purely hyperbolic isometries of $\mathbb{H}^{3}$ or as hyperbolic isometries of $\mathbb{H}^{2}$. We observe that $T_{M}(a)^{-1}=T_{M}(-a)$ and $T_{M}(a) T_{M}(b)=T_{M}(a+b)$, and similarly with $T_{L_{1}}(\cdot)$.

Theorem 3.1. Let $\varrho$ be an element of $\mathcal{Q F}(S)$, and let $\Psi(\varrho)=\left(l_{1}, l_{2}, \tau_{1}, \tau_{2}\right) \in$ $\left(\mathbb{R}^{+}\right)^{2} \times(\mathbb{C} / 2 \pi i)^{2}$. Define d by $\cosh d=\left(1+\cosh \frac{l_{1}}{2} \cosh \frac{l_{2}}{2}\right) /\left(\sinh \frac{l_{1}}{2} \sinh \frac{l_{2}}{2}\right)$. Then $\varrho$ is conjugate to the representation $p \circ \rho$, where $\rho: \pi_{1}(S) \longrightarrow S L(2, \mathbb{C})$ is defined by

$$
\begin{aligned}
\rho\left(\alpha_{1}\right) & =T_{L_{1}}\left(l_{1}\right), \\
\rho\left(\alpha_{2}\right) & =T_{M}(-d) T_{L_{1}}\left(l_{2}\right) T_{M}(d), \\
\rho\left(\beta_{1}\right) & =T_{L_{1}}\left(\tau_{1}\right) T_{M}(d) T_{L_{1}}\left(\tau_{2}\right) T_{M}(d) .
\end{aligned}
$$

Hence, we also have

$$
\rho\left(\beta_{2}\right)=\rho\left(\alpha_{1}^{-1} \beta_{1} \alpha_{2}\right)=T_{L_{1}}\left(\tau_{1}-l_{1}\right) T_{M}(d) T_{L_{1}}\left(\tau_{2}+l_{2}\right) T_{M}(d) .
$$

Proof. Given $l_{1}, l_{2} \in \mathbb{R}$, there exists, up to isometry, a unique right-angled hexagon with three alternate sides of lengths $l_{1} / 2, l_{2} / 2,0$. By trigonometry for right-angled hexagons, the length of the side opposite to that of length 0 is $d$, with $\cosh d$ equal to the expression in the statement.

In the Poincaré halfspace model of $\mathbb{H}^{3}$, we take two copies of this hexagon in the plane determined by the lines $L_{1}, M$, as in Figure 2. We denote by $L_{2}$ the oriented line containing the sides of length $l_{2} / 2$, so that $\mathbf{d}_{M}\left(L_{2}, L_{1}\right)=d$.

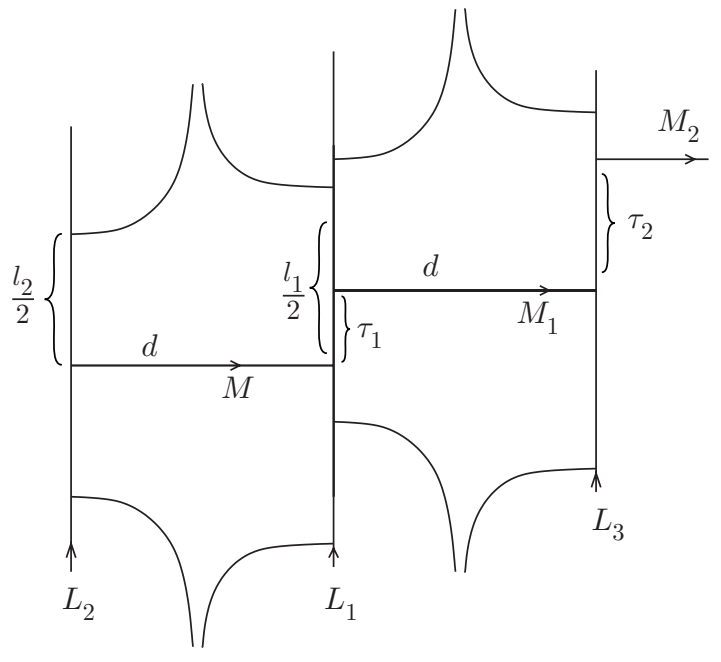

FIGURE 2 .

Let $M_{1}$ be the oriented line so that $\mathbf{d}_{L_{1}}\left(M, M_{1}\right)=\tau_{1}, L_{3}$ the oriented line so that $\mathbf{d}_{M_{1}}\left(L_{1}, L_{3}\right)=d$ and $M_{2}$ the oriented line so that $\mathbf{d}_{L_{3}}\left(M_{1}, M_{2}\right)=\tau_{2}$. We 
take another two copies of the hexagon in the plane determined by $L_{1}, M_{1}$, as in the figure. The given representation $\varrho$ is conjugate to a representation $\bar{\rho}$ which maps $\alpha_{1}$ to $\bar{T}_{L_{1}}\left(l_{1}\right)$ and $\alpha_{2}$ to $\bar{T}_{L_{2}}\left(l_{2}\right)$. Taking into account the definition of the twist parameters, $\bar{\rho}$ must map $\beta_{1}$ to the loxodromic element mapping the oriented lines $L_{2}, M$ into the lines $L_{3}, M_{2}$. This loxodromic element is the composition $\bar{T}_{L_{3}}\left(\tau_{2}\right) \bar{T}_{M_{1}}(d) \bar{T}_{L_{1}}\left(\tau_{1}\right) \bar{T}_{M}(d)$.

Observe that each of the lines $L_{2}, M, L_{1}, M_{1}, L_{3}$ is orthogonal to its two neighbours in the list. Therefore, we can easily express any loxodromic element whose axis is any of these lines as a composition of loxodromic elements with axes $M$ and $L_{1}$. In this way, we get

$$
\begin{aligned}
\bar{T}_{L_{2}}\left(l_{2}\right) & =\bar{T}_{M}(-d) \bar{T}_{L_{1}}\left(l_{2}\right) \bar{T}_{M}(d) \\
\bar{T}_{L_{3}}\left(\tau_{2}\right) \bar{T}_{M_{1}}(d) \bar{T}_{L_{1}}\left(\tau_{1}\right) \bar{T}_{M}(d) & =\bar{T}_{M_{1}}(d) \bar{T}_{L_{1}}\left(\tau_{2}\right) \bar{T}_{M_{1}}(-d) \bar{T}_{M_{1}}(d) \bar{T}_{L_{1}}\left(\tau_{1}\right) \bar{T}_{M}(d) \\
& =\bar{T}_{L_{1}}\left(\tau_{1}\right) \bar{T}_{M}(d) \bar{T}_{L_{1}}\left(-\tau_{1}\right) \bar{T}_{L_{1}}\left(\tau_{2}\right) \bar{T}_{L_{1}}\left(\tau_{1}\right) \bar{T}_{M}(d) \\
& =\bar{T}_{L_{1}}\left(\tau_{1}\right) \bar{T}_{M}(d) \bar{T}_{L_{1}}\left(\tau_{2}\right) \bar{T}_{M}(d) .
\end{aligned}
$$

Finally, since $\pi_{1}(S)$ is a free group, this representation lifts to the representation $\rho: \pi_{1}(S) \rightarrow S L(2, \mathbb{C})$ given in the statement; see [18].

Change of Fenchel-Nielsen coordinates. The curves $\left\{\alpha_{1}, \alpha_{2}\right\}$ and $\left\{\beta_{1}, \beta_{2}\right\}$ are symmetric in the surface. More precisely, there exists a homeomorphism, $r: S \rightarrow S$, see Figure 3, so that the induced homomorphism $r_{*}$ of the fundamental groups maps $\alpha_{1} \mapsto \beta_{1}, \alpha_{2} \mapsto \beta_{2}, \beta_{1} \mapsto \alpha_{1}, \beta_{2} \mapsto \alpha_{2}$. We can also check that $\delta_{1} \mapsto \delta_{3}=$ $\alpha_{1} \beta_{2} \alpha_{1}^{-1} \beta_{2}^{-1}$ and $\delta_{2} \mapsto \beta_{1} \alpha_{1}^{-1} \delta_{2}^{-1} \alpha_{1} \beta_{1}^{-1}$, which is a conjugate of $\delta_{2}^{-1}$. Note that $r$ reverses the orientation of the surface.

We can work with Fenchel-Nielsen coordinates with respect to the pants decomposition $\left\{\beta_{1}, \beta_{2}\right\}$ and dual system $\left\{\delta_{3}, \delta_{2}\right\}$. Let $\varrho$ be an element in $\mathcal{Q F}(S)$, and $\Psi^{\beta}(\varrho)=\left(\lambda_{\beta_{1}}, \lambda_{\beta_{2}}, \tau_{\beta_{1}}, \tau_{\beta_{2}}\right)$ its complex Fenchel-Nielsen coordinates with respect to this new pants decomposition. Assume that the lengths $\lambda_{\beta_{1}}=l_{\beta_{1}}, \lambda_{\beta_{2}}=l_{\beta_{2}}$ are real. Then, proceeding as in Theorem 3.1. we can normalize $\varrho$ to a representation $p \circ \rho^{\beta}$, where $\rho^{\beta}: \pi_{1}(S) \longrightarrow S L(2, \mathbb{C})$, defined by

$$
\begin{aligned}
\rho^{\beta}\left(\beta_{1}\right) & =T_{L_{1}}\left(l_{\beta_{1}}\right), \\
\rho^{\beta}\left(\beta_{2}\right) & =T_{M}\left(d^{\beta}\right) T_{L_{1}}\left(l_{\beta_{2}}\right) T_{M}\left(-d^{\beta}\right), \\
\rho^{\beta}\left(\alpha_{1}\right) & =T_{L_{1}}\left(-\tau_{\beta_{1}}\right) T_{M}\left(-d^{\beta}\right) T_{L_{1}}\left(-\tau_{\beta_{2}}\right) T_{M}\left(-d^{\beta}\right), \\
\rho^{\beta}\left(\alpha_{2}\right)=\rho^{\beta}\left(\beta_{1}^{-1} \alpha_{1} \beta_{2}\right) & =T_{L_{1}}\left(-\tau_{\beta_{1}}-l_{\beta_{1}}\right) T_{M}\left(-d^{\beta}\right) T_{L_{1}}\left(-\tau_{2}+l_{\beta_{2}}\right) T_{M}\left(-d^{\beta}\right),
\end{aligned}
$$

where $d^{\beta}$ is defined by $\cosh d^{\beta}=\left(1+\cosh \frac{l_{\beta_{1}}}{2} \cosh \frac{l_{\beta_{2}}}{2}\right) /\left(\sinh \frac{l_{\beta_{1}}}{2} \sinh \frac{l_{\beta_{2}}}{2}\right)$. Note that the fact that $r$ reverses orientation produces a change of sign in the twist parameters.

Usually, we will use the complex Fenchel-Nielsen coordinates with respect to $\left\{\alpha_{1}, \alpha_{2}\right\}$ and the dual system $\left\{\delta_{1}, \delta_{2}\right\}$. We shall refer to these as $\alpha$-coordinates. We will refer to the complex Fenchel-Nielsen coordinates with respect to $\left\{\beta_{1}, \beta_{2}\right\}$ and the dual system $\left\{\delta_{3}, \delta_{2}\right\}$ as $\beta$-coordinates.

Remark. We can regard Theorem 3.1 and the above paragraph (generalized to complex values of the lengths of $\alpha_{i}$ or $\beta_{i}$ ) as defining two different charts of the representation variety $\mathcal{R}$ with domain $\mathbb{R}^{2} \times(\mathbb{C} / 2 \pi i)^{2}$. The image of both charts strictly contains $\mathcal{Q} \mathcal{F}$ but not its closure in $\mathcal{R}$, since cusp groups in $\partial \mathcal{Q F}$ with $\alpha_{1}$ or $\alpha_{2}$ pinched are not in the image of the first chart, whereas cusp groups with $\beta_{1}$ 


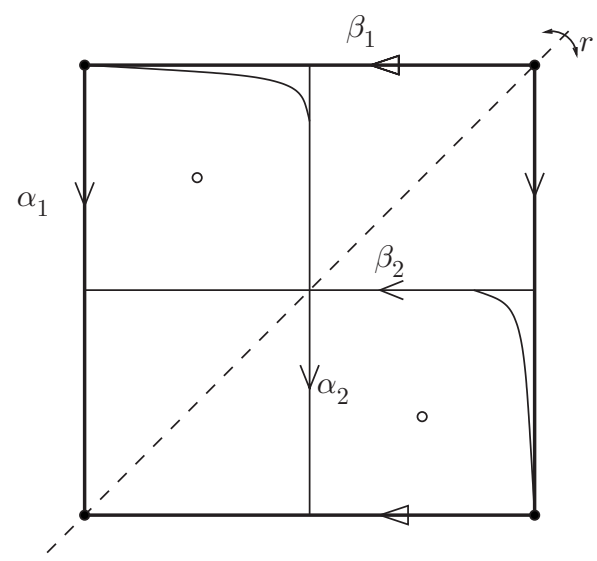

Figure 3. Change of Fenchel-Nielsen coordinates.

or $\beta_{2}$ pinched are not in the image of the second one. Throughout the paper we will identify points in $\mathbb{R}^{2} \times(\mathbb{C} / 2 \pi i)^{2}$ with their image representations obtained as in Theorem 3.1

3.1. Some formulae. From now on we will mostly use the notation $\rho \gamma$ instead of $\rho(\gamma)$. The decomposition of $\rho \beta_{1}, \rho \beta_{2}$ as a product of four loxodromics along two orthogonal axes is convenient for various computations. In this section we compute the real and imaginary parts of the traces of $\rho \beta_{1}, \rho \beta_{2}$. The expression that we obtain involves certain quantities in terms of $V_{1}, e_{1}, V_{2}, e_{2}$ whose geometric meaning is explained in Figure 4, which we call the Main Figure. Despite the fact that we are working with isometries of $\mathbb{H}^{3}$, the Main Figure is entirely a figure in $\mathbb{H}^{2}$.

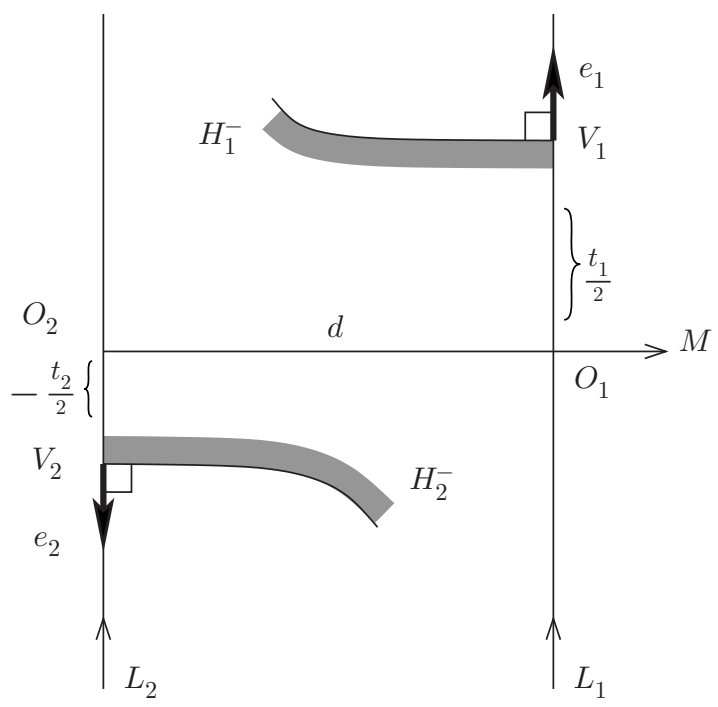

Figure 4. Main Figure. 
The Main Figure. Let $d>0$ and $t_{1}, t_{2} \in \mathbb{R}$. Let $L_{1}, L_{2}$ be oriented hyperbolic lines and $M$ their oriented common perpendicular, satisfying $\mathbf{d}_{M}\left(L_{2}, L_{1}\right)=d$. Denote by $O_{1}, O_{2}$ the intersection points of $L_{1}, M$ and $L_{2}, M$, respectively. Let $V_{1}=V_{1}\left(t_{1}\right)$ be the point on $L_{1}$ at signed distance $t_{1} / 2$ from $O_{1}$ and let $V_{2}=V_{2}\left(t_{2}\right)$ be the point on $L_{2}$ at signed distance $-t_{2} / 2$ from $O_{2}$. Consider the halfplane $H_{1}^{-}$orthogonal to $L_{1}$ at $V_{1}$ and containing the initial endpoint of $L_{1}$ (the one shaded in the figure), and let $e_{1}=e_{1}\left(t_{1}\right)$ be its outward unit normal. In the figure, this vector is represented as a tangent vector at the point $V_{1}$, but it must be thought as the vector in $\mathbb{R}^{3}$ defined as explained in Section 2.1 Similarly, consider the halfplane $H_{2}^{-}$orthogonal to $L_{2}$ at $V_{2}$ containing the final endpoint of $L_{2}$ with outward unit normal $e_{2}=e_{2}\left(t_{2}\right)$.

Remark 1. Much geometric information can be obtained by just looking at this figure. For instance, observe that $\left\langle e_{1}, V_{2}\right\rangle=0$ if and only if $V_{2}$ is in the line $H_{1}$ orthogonal to $L_{1}$ through $V_{1} ;\left\langle e_{1}, V_{2}\right\rangle<0$ if the point $V_{2}$ is contained in the interior of the halfplane $H_{1}^{-}$, and $\left\langle e_{1}, V_{2}\right\rangle>0$ if $V_{2}$ is contained in the interior of the halfplane $H_{1}^{+}$, and similarly for $\left\langle V_{1}, e_{2}\right\rangle$. As a consequence, $\left\langle e_{1}, V_{2}\right\rangle=\left\langle V_{1}, e_{2}\right\rangle=0$ if and only if $t_{1}=t_{2}=0$.

Remark 2. If $\tau_{1}=t_{1}, \tau_{2}=t_{2}$ are real, then the axis of the isometry $p \circ \rho\left(\beta_{1}\right)=$ $\bar{T}_{L_{1}}\left(t_{1}\right) \bar{T}_{M}(d) \bar{T}_{L_{1}}\left(t_{2}\right) \bar{T}_{M}(d)$ is the line joining $V_{1}\left(t_{1}\right)$ and $V_{2}\left(t_{2}\right)$. Indeed, we can express $\bar{T}_{L_{1}}\left(t_{2}\right) \bar{T}_{M}(d)$ as a product of involutions:

$$
I_{V_{1}\left(t_{2}\right)} I_{O_{1}} I_{O_{1}} I_{O}=I_{V_{1}\left(t_{2}\right)} I_{O}=I_{O} I_{V_{2}\left(t_{2}\right)},
$$

where $O$ is the point on $M$ equidistant to $L_{1}$ and $L_{2}$ and $I_{P}$ denotes rotation by $\pi$ about the point $P$. Doing similarly with $\bar{T}_{L_{1}}\left(t_{1}\right) \bar{T}_{M}(d)$, we get that

$$
\bar{T}_{L_{1}}\left(t_{1}\right) \bar{T}_{M}(d) \bar{T}_{L_{1}}\left(t_{2}\right) \bar{T}_{M}(d)=I_{V_{1}\left(t_{1}\right)} I_{V_{2}\left(t_{2}\right)} \text {. }
$$

The following proposition is a formula for decomposing the trace of $\rho \beta_{1}=$ $T_{L_{1}}\left(\tau_{1}\right) T_{M}(d) T_{L_{1}}\left(\tau_{2}\right) T_{M}(d)$ into its real and imaginary parts. If $\tau_{1}, \tau_{2} \in \mathbb{R}$, we can check that $\operatorname{tr} \rho \beta_{1}=-2\left\langle V_{1}, V_{2}\right\rangle$.

Proposition 3.2. Consider $d>0$, and let $\tau_{1}=t_{1}+i \theta_{1}$ and $\tau_{2}=t_{2}+i \theta_{2}$ be two complex numbers. With the above notation, we have

$$
\begin{aligned}
\operatorname{tr}\left(T_{L_{1}}\left(\tau_{1}\right) T_{M}(d) T_{L_{1}}\left(\tau_{2}\right) T_{M}(d)\right) & \\
= & -2\left(\cos \frac{\theta_{1}}{2}, i \sin \frac{\theta_{1}}{2}\right)\left(\begin{array}{ll}
\left\langle V_{1}, V_{2}\right\rangle & \left\langle V_{1}, e_{2}\right\rangle \\
\left\langle e_{1}, V_{2}\right\rangle & \left\langle e_{1}, e_{2}\right\rangle
\end{array}\right)\left(\begin{array}{c}
\cos \frac{\theta_{2}}{2} \\
i \sin \frac{\theta_{2}}{2}
\end{array}\right) \\
= & -2\left(\cos \frac{\theta_{1}}{2} \cos \frac{\theta_{2}}{2}\left\langle V_{1}, V_{2}\right\rangle-\sin \frac{\theta_{1}}{2} \sin \frac{\theta_{2}}{2}\left\langle e_{1}, e_{2}\right\rangle\right) \\
& -2 i\left(\cos \frac{\theta_{1}}{2} \sin \frac{\theta_{2}}{2}\left\langle V_{1}, e_{2}\right\rangle+\sin \frac{\theta_{1}}{2} \cos \frac{\theta_{2}}{2}\left\langle e_{1}, V_{2}\right\rangle\right) .
\end{aligned}
$$

For the proof of this proposition we use the following lemma.

Lemma 3.3. Let $a, b, a_{i}, b_{i} \in \mathbb{C}$ and let $T_{L_{1}}(a), T_{M}(a)$ be as above. With the notation $\mathrm{t}(a)=2 \cosh (a / 2)$ :

(a) $\operatorname{tr}\left(T_{L_{1}}(a) T_{M}(b)\right)=\frac{1}{2} \mathrm{t}(a) \mathrm{t}(b)$, and

(b) $\operatorname{tr}\left(T_{L_{1}}\left(a_{1}\right) T_{M}\left(b_{1}\right) T_{L_{1}}\left(a_{2}\right) T_{M}\left(b_{2}\right)\right)$

$$
=\frac{1}{4} \mathrm{t}\left(a_{1}\right) \mathrm{t}\left(b_{1}\right) \mathrm{t}\left(a_{2}\right) \mathrm{t}\left(b_{2}\right)-\frac{1}{2} \mathrm{t}\left(a_{1}-a_{2}\right) \mathrm{t}\left(b_{1}-b_{2}\right) .
$$

Proof. Notice that $\mathrm{t}(a)=2 \cosh (a / 2)=\operatorname{tr}\left(T_{L_{1}}(a)\right)=\operatorname{tr}\left(T_{M}(a)\right)$. One can prove (a) by direct computation. For (b), use the trace formulae $\operatorname{tr}(A B)=\operatorname{tr}(B A)$ and $\operatorname{tr}(A B)=\operatorname{tr} A \operatorname{tr} B-\operatorname{tr}\left(A B^{-1}\right)$ and (a). 
Proof of Proposition [3.2. Applying Lemma 3.3 (b) and expanding hyperbolic cosines of sums, we get

$$
\begin{aligned}
& \operatorname{tr}\left(T_{L_{1}}\left(\tau_{1}\right) T_{M}(d) T_{L_{1}}\left(\tau_{2}\right) T_{M}(d)\right) \\
& \quad=A \cos \frac{\theta_{1}}{2} \cos \frac{\theta_{2}}{2}-B \sin \frac{\theta_{1}}{2} \sin \frac{\theta_{2}}{2}+i\left(C \cos \frac{\theta_{1}}{2} \sin \frac{\theta_{2}}{2}+D \sin \frac{\theta_{1}}{2} \cos \frac{\theta_{2}}{2}\right),
\end{aligned}
$$

where

$$
\begin{aligned}
A & =t^{2}(d) \cosh \frac{t_{1}}{2} \cosh \frac{t_{2}}{2}-2 \cosh \frac{t_{1}-t_{2}}{2}, \\
B & =t^{2}(d) \sinh \frac{t_{1}}{2} \sinh \frac{t_{2}}{2}+2 \cosh \frac{t_{1}-t_{2}}{2} \\
C & =t^{2}(d) \cosh \frac{t_{1}}{2} \sinh \frac{t_{2}}{2}+2 \sinh \frac{t_{1}-t_{2}}{2} \\
D & =t^{2}(d) \sinh \frac{t_{1}}{2} \cosh \frac{t_{2}}{2}-2 \sinh \frac{t_{1}-t_{2}}{2}
\end{aligned}
$$

We work in the hyperboloid model of $\mathbb{H}^{2}$, where we normalize the Main Figure so that $O_{1}, O_{2}, V_{1}, V_{2}, e_{1}, e_{2}$ are the following vectors in $\mathbb{R}^{3}$ :

$$
\begin{aligned}
O_{2}=(0,0,1), & O_{1}=(\sinh d, 0, \cosh d), \\
V_{2}=\left(0,-\sinh \frac{t_{2}}{2}, \cosh \frac{t_{2}}{2}\right), & V_{1}=\left(\sinh d \cosh \frac{t_{1}}{2}, \sinh \frac{t_{1}}{2}, \cosh d \cosh \frac{t_{1}}{2}\right), \\
e_{2}=\left(0,-\cosh \frac{t_{2}}{2}, \sinh \frac{t_{2}}{2}\right), & e_{1}=\left(\sinh d \sinh \frac{t_{1}}{2}, \cosh \frac{t_{1}}{2}, \cosh d \sinh \frac{t_{1}}{2}\right) .
\end{aligned}
$$

Computing the inner products, we find that

$$
\left\langle V_{1}, V_{2}\right\rangle=-\sinh \frac{t_{1}}{2} \sinh \frac{t_{2}}{2}-\cosh d \cosh \frac{t_{1}}{2} \cosh \frac{t_{2}}{2}=-A / 2,
$$

and similarly $\left\langle e_{1}, e_{2}\right\rangle=-B / 2,\left\langle V_{1}, e_{2}\right\rangle=-C / 2$ and $\left\langle e_{1}, V_{2}\right\rangle=-D / 2$.

Remark 3. It will be convenient to list the following expressions:

$$
\begin{aligned}
& -\left\langle V_{1}\left(t_{1}\right), V_{2}\left(t_{2}\right)\right\rangle=\cosh d \cosh \frac{t_{1}}{2} \cosh \frac{t_{2}}{2}+\sinh \frac{t_{1}}{2} \sinh \frac{t_{2}}{2}, \\
& -\left\langle e_{1}\left(t_{1}\right), V_{2}\left(t_{2}\right)\right\rangle=\cosh d \sinh \frac{t_{1}}{2} \cosh \frac{t_{2}}{2}+\cosh \frac{t_{1}}{2} \sinh \frac{t_{2}}{2}, \\
& -\left\langle V_{1}\left(t_{1}\right), e_{2}\left(t_{2}\right)\right\rangle=\cosh d \cosh \frac{t_{1}}{2} \sinh \frac{t_{2}}{2}+\sinh \frac{t_{1}}{2} \cosh \frac{t_{2}}{2}, \\
& -\left\langle e_{1}\left(t_{1}\right), e_{2}\left(t_{2}\right)\right\rangle=\cosh d \sinh \frac{t_{1}}{2} \sinh \frac{t_{2}}{2}+\cosh \frac{t_{1}}{2} \cosh \frac{t_{2}}{2} .
\end{aligned}
$$

\section{Simplices of Minima}

In this section we shall find the equations for some lines and simplices of minima in the Teichmüller space of the twice punctured torus. We start with some general results.

4.1. Kerckhoff's theory. We recall the main result of Kerckhoff that leads to the definition of lines of minima ([15], see also 20], Section 4). The time $t$ left earthquake 14, 23] along a lamination $\mu \in \mathcal{M L}(S)$ is a real analytic map $\mathcal{E}_{\mu}(t)$ : $\operatorname{Teich}(S) \rightarrow \operatorname{Teich}(S)$ which generalizes the classical Fenchel-Nielsen twist. If $\rho \in$ $\operatorname{Teich}(S)$, we denote by $\mathcal{E}_{\mu}(\rho)$ the earthquake path $\mathcal{E}_{\mu}(t)(\rho), t \in \mathbb{R}$. This flow induces a tangent vector field $\frac{\partial}{\partial t_{\mu}}$ on $\operatorname{Teich}(S)$. In [14, Kerckhoff showed that if $\nu \in \mathcal{M L}$, then the length $l_{\nu}$ is a real analytic function of $t$ along $\mathcal{E}_{\mu}(\rho)$, strictly convex if 
$i(\mu, \nu)>0$ and constant otherwise. If $\mu, \nu$ are curves, the derivative of the length of $\nu$ along the earthquake path is

$$
\frac{\partial l_{\nu}}{\partial t_{\mu}}=\sum \cos \psi_{i}
$$

where $\psi_{i}$ is the angle from $\nu$ to $\mu$ at a point of intersection, and the sum is over all the intersection points of $\mu, \nu$. From this formula, one obtains Wolpert's antisymmetry formula

$$
\frac{\partial l_{\nu}}{\partial t_{\mu}}=-\frac{\partial l_{\mu}}{\partial t_{\nu}}
$$

The following is the fundamental theorem of [15]. We say that two measured laminations $\mu, \nu$ fill up the surface if for any other lamination $\xi$ we have $i(\mu, \xi)+$ $i(\nu, \xi) \neq 0$.

Theorem 4.1 ([15], Theorem 1.2). Suppose $\mu$ and $\nu$ fill up $S$. Then for every $k \in \mathbb{R}^{+}$the function $f_{k}=l_{\mu}+k l_{\nu}$ has a unique critical point on $\operatorname{Teich}(S)$ which is a global minimum for $f_{k}$.

The set of minimum points of $f_{k}$ for $k \in \mathbb{R}^{+}$is called the line of minima of $\mu, \nu$, denoted $\mathcal{L}_{\mu, \nu}$.

The following theorem of Kerckhoff shows that for fixed $\mu \in \mathcal{M L}$, the lines of minima $\mathcal{L}_{\mu, \nu}$ foliate Teich $(S)$. Suppose that $\mu \in \mathcal{M L}$ and denote by $\mathcal{M} \mathcal{L}_{\mu}$ the set of $\nu \in \mathcal{M L}$ such that $\mu$ and $\nu$ fill up $S$. Fix a continuous section $j: \operatorname{P} \mathcal{M L} \rightarrow \mathcal{M L}$.

Theorem 4.2 ([15], Theorem 2.1). Let $\mu \in \mathcal{M L}$. The map $\Phi_{\mu}: \mathrm{PM}_{\mathcal{L}} \mathcal{L}_{\mu} \times \mathbb{R}^{+} \rightarrow$ $\operatorname{Teich}(S)$, sending $([\nu], k)$ to the point where $l_{\mu}+k l_{j([\nu])}$ attains its minimum, is a homeomorphism.

We have also the following useful characterization of lines of minima.

Proposition 4.3. Let $\mu, \nu \in \mathcal{M L}$ with $[\mu] \neq[\nu]$ and $\rho \in \operatorname{Teich}(S)$. Then there exists $k \in \mathbb{R}$ with $\left.\frac{\partial}{\partial t_{\mu}}\right|_{\rho}=-\left.k \frac{\partial}{\partial t_{\nu}}\right|_{\rho}$ if and only if $\mu, \nu$ fill up $S, k$ is positive, and $\rho$ is the unique minimum of $f_{k}$, and hence in $\mathcal{L}_{\mu, \nu}$.

This result is also based on [15]; see 20], Section 4, for the proof.

Let $\mathcal{A}=\left\{\alpha_{1}, \ldots, \alpha_{n}\right\}$ and $\mathcal{B}=\left\{\beta_{1}, \ldots, \beta_{m}\right\}$ be two systems of disjoint, simple closed curves on the surface $S$. We define the (open) simplex of minima $\mathcal{S}_{\mathcal{A}, \mathcal{B}}$ to be the union of lines of minima $\mathcal{L}_{\mu, \nu}$, where $\mu=\sum a_{i} \alpha_{i}, \nu=\sum b_{i} \beta_{i}$ are linear combinations of $\alpha_{i}, \beta_{j}$, respectively, with all the coefficients positive. It follows from Proposition 4.4 below that the closure $\overline{\mathcal{S}}_{\mathcal{A}, \mathcal{B}}$ of $\mathcal{S}_{\mathcal{A}, \mathcal{B}}$ in Teichmüller space is the union of lines of minima $\mathcal{L}_{\mu, \nu}$ with $\mu$ and $\nu$ as before but with $a_{i}, b_{i} \geq 0$, and such that the curves with positive coefficients still fill up the surface.

We can regard $\mathcal{S}_{\mathcal{A}, \mathcal{B}}$ as the image of the following affine simplex. Consider independent points $A_{1}, \ldots, A_{n}, B_{1}, \ldots, B_{m}$ in $\mathbb{R}^{n+m-1}$, and let $Q_{\mathcal{A}, \mathcal{B}}$ be the open simplex they generate. Thus, a point $q$ in $Q_{\mathcal{A}, \mathcal{B}}$ is of the form $(1-t)\left(\sum_{i} a_{i} A_{i}\right)+$ $t\left(\sum_{j} b_{j} B_{j}\right)$, with $0<t, a_{i}, b_{j}<1, \sum a_{i}=1, \sum b_{j}=1$. Let $\hat{Q}$ be the set of points $q$ of the same form as before but with $a_{i}, b_{i} \geq 0$ and such that the set of curves $\left\{\alpha_{i}, \beta_{j} \mid a_{i}>0, b_{j}>0\right\}$ fills up the surface. Finally, define the map $\Phi: \hat{Q}_{\mathcal{A}, \mathcal{B}} \rightarrow \operatorname{Teich}(S)$ by assigning to $(1-t)\left(\sum_{i} a_{i} A_{i}\right)+t\left(\sum_{j} b_{j} B_{j}\right)$ the unique minimum of the function $(1-t)\left(\sum_{i} a_{i} l_{\alpha_{i}}\right)+t\left(\sum_{j} b_{j} l_{\beta_{j}}\right)$.

Essentially the same reasoning as in parts (I), (II) of Theorem 2.1 of [15] proves 
Proposition 4.4. The map $\Phi: \hat{Q}_{\alpha, \mathcal{B}} \rightarrow \operatorname{Teich}(S)$ is continuous and proper.

In consequence, as asserted, the closure of $\mathcal{S}_{\mathcal{A}, \mathcal{B}}$ in Teichmüller space is the closed simplex $\overline{\mathcal{S}}_{\mathcal{A}, \mathcal{B}}$. It also follows that simplices of minima are connected subsets of Teich $(S)$. (In [3], we study the closure of $\overline{\mathcal{S}}_{\mathcal{A}, \mathcal{B}}$ in Thurston's compactification of Teichmüller space, and show that the limit points of the line of minima $\mathcal{L}_{\mu, \nu}$ are the barycentres $\left[\alpha_{1}+\cdots+\alpha_{n}\right]$ and $\left[\beta_{1}+\cdots+\beta_{n}\right]$.)

For pants decompositions $\mathcal{A}=\left\{\alpha_{1}, \ldots, \alpha_{n}\right\}, \mathcal{B}=\left\{\beta_{1}, \ldots, \beta_{n}\right\}$ of $S$, we define the matrix $M=M(\mathcal{A}, \mathcal{B}, \rho)$ as

$$
M(\mathcal{A}, \mathcal{B}, \rho)=\left(\begin{array}{ccc}
\left.\frac{\partial l_{\beta_{1}}}{\partial t_{\alpha_{1}}}\right|_{\rho} & \cdots & \left.\frac{\partial l_{\beta_{1}}}{\partial t_{\alpha_{n}}}\right|_{\rho} \\
\vdots & & \vdots \\
\left.\frac{\partial l_{\beta_{n}}}{\partial t_{\alpha_{1}}}\right|_{\rho} & \cdots & \left.\frac{\partial l_{\beta_{n}}}{\partial t_{\alpha_{n}}}\right|_{\rho}
\end{array}\right) .
$$

Observe that a vector $\left(a_{1}, \ldots, a_{n}\right)^{T}$ is a right nullvector of $M$ if and only if $\frac{\partial l_{\beta_{i}}}{\partial t_{\mu}}=0$ for all $i$, where $\mu$ is the formal linear combination $a_{1} \alpha_{1}+\cdots+a_{n} \alpha_{n}$. Similarly, by using Wolpert's antisymmetry formula, a vector $\left(b_{1}, \ldots, b_{n}\right)$ is a left nullvector of $M$ if and only if $\frac{\partial l_{\alpha_{i}}}{\partial t_{\nu}}=0$ for all $i$, where $\nu$ is the formal linear combination $b_{1} \beta_{1}+\cdots+b_{n} \beta_{n}$. Writing $\frac{\partial}{\partial t_{\mu}}$ in terms of the coordinates induced by FenchelNielsen coordinates $l_{\beta_{i}}, t_{\beta_{i}}$, we have

$$
\frac{\partial}{\partial t_{\mu}}=\sum \frac{\partial l_{\beta_{i}}}{\partial t_{\mu}} \frac{\partial}{\partial l_{\beta_{i}}}+\sum \frac{\partial t_{\beta_{i}}}{\partial t_{\mu}} \frac{\partial}{\partial t_{\beta_{i}}}
$$

Thus, if $\left(a_{1}, \ldots, a_{n}\right)^{T}$ is a right nullvector of $M$, we get that the first group of summands is zero. As a consequence, applying this expression to the functions $l_{\alpha_{i}}$, we get that the vector $\left(\frac{\partial t_{\beta_{1}}}{\partial t_{\mu}}, \ldots, \frac{\partial t_{\beta_{n}}}{\partial t_{\mu}}\right)$ is a left nullvector of $M$.

Using the matrix $M$, we can give yet another characterization of rational lines of minima.

Proposition 4.5. Let $\mu=\sum a_{i} \alpha_{i}, \nu=\sum b_{i} \beta_{i}$; a point $\rho$ is in the line of minima $\mathcal{L}_{\mu, \nu}$ if and only if $\left(a_{1}, \ldots a_{n}\right)^{T}$ is a right nullvector of $M,\left(b_{1}, \ldots b_{n}\right)$ is a left nullvector, and there exists $k>0$ so that $-k b_{i}=\left.\frac{\partial t_{\beta_{i}}}{\partial t_{\mu}}\right|_{\rho}$, for all $i$. In particular, if $\rho \in \mathcal{L}_{\mu, \nu}$, then $\operatorname{det} M=0$.

Proof. If $\rho \in \mathcal{L}_{\mu, \nu}$, then, by Proposition 4.3 , there exists a $k>0$ so that

$$
\left.\frac{\partial}{\partial t_{\mu}}\right|_{\rho}=-\left.k \frac{\partial}{\partial t_{\nu}}\right|_{\rho}
$$

Since $\frac{\partial l_{\alpha_{i}}}{\partial t_{\mu}}=0$, then (2) gives $\frac{\partial l_{\alpha_{i}}}{\partial t_{\nu}}=0$, so that $\left(b_{1}, \ldots, b_{n}\right)$ is a left nullvector of $M$. Similarly we obtain that $\left(a_{1}, \ldots, a_{n}\right)^{T}$ is a right nullvector of $M$. For the relation between these nullvectors, we write $\frac{\partial}{\partial t_{\mu}}$ as in (1); since $\left(a_{1}, \ldots, a_{n}\right)^{T}$ is a right nullvector, $\partial / \partial t_{\mu}$ is a combination of $\left\{\partial / \partial t_{\beta_{j}}\right\}$. Then, using (2), we obtain the relation in the statement.

For the converse, we apply the hypothesis to the expression (1) to obtain (2). Then, by Proposition $4.3, \rho \in \mathcal{L}_{\mu, \nu}$.

The map $\Phi$ is not always injective. In the following proposition we study some necessary and sufficient conditions for injectivity. This result will be used to compute the simplex of minima $\overline{\mathcal{S}}_{\alpha_{1}, \alpha_{2} ; \beta_{1}, \beta_{2}}$. 
Proposition 4.6 (cf. [20, Corollary 6.3). Let $\mathcal{A}=\left\{\alpha_{1}, \ldots, \alpha_{n}\right\}, \mathcal{B}=\left\{\beta_{1}, \ldots, \beta_{n}\right\}$ be two pants decompositions of $S$ and $\rho \in \operatorname{Teich}(S)$ such that $\operatorname{det} M(\mathcal{A}, \mathcal{B}, \rho)=0$.

(a) Suppose $\operatorname{rk} M=n-1$. Then $\rho \in \operatorname{Im} \Phi$ if and only if the adjoint matrix of $M$ has all its entries of the same sign (with possibly some entries vanishing). Moreover $\Phi^{-1}(\rho)$ consists exactly of one point. In particular, if $\operatorname{rk} M=n-1$ on $\operatorname{Im} \Phi$, then $\Phi$ is a homeomorphism onto its image.

(b) Suppose $\operatorname{rk} M=n_{0}<n-1$ and $\rho \in \mathcal{L}_{\mu, \nu} \subset \operatorname{Im} \Phi$, with $a_{i}>0$ and $b_{i}>0$ for all $i$. Then $\Phi^{-1}(\rho)$ contains a subset of dimension $n-1-n_{0}$.

Proof. (a) If $\rho \in \operatorname{Im} \Phi$, then it is in a line of minima $\mathcal{L}_{\mu, \nu}$, with $\mu=\sum a_{i} \alpha_{i}, \nu=$ $\sum b_{i} \beta_{i}$, with $a_{i}, b_{i} \geq 0$. Since $(\operatorname{Adj} M) M=M(\operatorname{Adj} M)=\operatorname{det} M$, every row of $\operatorname{Adj} M$ is a left nullvector of $M$, and every column of $\operatorname{Adj} M$ is a right nullvector of $M$. Since $M$ has rank $n-1$, all left nullvectors are proportional, and so are all right nullvectors. By Proposition 4.5, $\left(b_{1}, \ldots, b_{n}\right)$ and $\left(a_{1}, \ldots, a_{n}\right)^{T}$ are positive left and right nullvectors. We conclude that all the entries of $\operatorname{Adj} M$ have the same sign.

For the converse, take a non-zero row $\left(b_{1}, \ldots, b_{n}\right)$ and column $\left(a_{1}, \ldots, a_{n}\right)^{T}$ of $\operatorname{Adj} M$ (or their opposite, if $\operatorname{Adj} M$ has its entries negative), and consider $\mu=$ $\sum a_{i} \alpha_{i}, \nu=\sum b_{i} \beta_{i}$. Since $\left(a_{1}, \ldots, a_{n}\right)^{T}$ is a right nullvector of $M,\left(\frac{\partial t_{\beta_{1}}}{\partial t_{\mu}}, \ldots, \frac{\partial t_{\beta_{n}}}{\partial t_{\mu}}\right)$ is a left nullvector. Since $\operatorname{rk} M=n-1$, this nullvector is proportional to $\left(b_{1}, \ldots, b_{n}\right)$. By Proposition 4.5, $\rho$ is in the line of minima $\mathcal{L}_{\mu, \nu}$. For injectivity, first note that each segment of $Q_{\mathcal{A}, \mathcal{B}}$ with vertices $\sum a_{i} A_{i}, \sum b_{i} B_{i}$ maps bijectively to a line of minima. Second, if $\Phi^{-1}(\rho)$ is in two different lines, then the matrix $M$ has two non-proportional nullvectors.

(b) Consider $\rho \in \mathcal{L}_{\mu, \nu}$, with $a_{i}, b_{j}>0$. By Proposition 4.5, there exists $k>0$ so that $-k b_{i}=\frac{\partial t_{\beta_{i}}}{\partial t_{\mu}}$. Thus, we can regard $\left(b_{1}, \ldots, b_{n}\right)$ as the image of $\left(a_{1}, \ldots, a_{n}\right)^{T}$ by the linear map $G$ defined by the matrix $(-1 / k)\left(\frac{\partial t_{\beta_{i}}}{\partial t_{\alpha_{j}}}\right)$. Denote by $R$ the set of right nullvectors of $M$ and by $C^{+}$the set of vectors of $\mathbb{R}^{n}$ with non-negative coordinates. Since $\left(a_{1}, \ldots, a_{n}\right)^{T}$ is a nullvector with all coordinates positive, $R$ intersects the interior of $C^{+}$and therefore $R \cap C^{+}$is a cone of dimension $n-n_{0}$. The map $G$ restricted to $R$ is injective because $\left(a_{1}^{\prime}, \ldots, a_{n}^{\prime}\right)^{T}$ being in the kernel of $G$ is equivalent to $\frac{\partial t_{\beta_{i}}}{\partial t_{\mu^{\prime}}}=0$ for all $i$, where $\mu^{\prime}=a_{1}^{\prime} \alpha_{1}+\cdots+a_{n}^{\prime} \alpha_{n}$. In this case, since $\left(a_{1}^{\prime}, \ldots, a_{n}^{\prime}\right)^{T}$ is a right nullvector, we have that $\frac{\partial}{\partial t_{\mu^{\prime}}}=0$, which forces $a_{1}^{\prime}=\cdots=a_{n}^{\prime}=0$. Hence, $G(R)$ has dimension $n-n_{0}$ and contains $\left(b_{1}, \ldots, b_{n}\right)$, which has all its coordinates positive, and so $G(R) \cap C^{+}$is a cone of dimension $n-n_{0}$. Then, positive right nullvectors near $\left(a_{1}, \ldots a_{n}\right)^{T}$ are mapped to left nullvectors near $\left(b_{1}, \ldots b_{n}\right)$, which will be also positive. By Proposition 4.5 we obtain the result.

By Kerckhoff's derivative formula, the entries of the matrix $M$ have geometric meaning in terms of the angles between the curves $\alpha_{i}$ and $\beta_{j}$. This will be useful for some proofs. At other times it is easier to work with traces rather than lengths. By differentiating the relation between trace and length we obtain the following expression for the matrix $\left.M\right|_{\rho}$ :

$$
M=\left(\begin{array}{ccc}
\frac{1}{\sinh \left(l_{\beta_{1} / 2}\right)} & \ldots & 0 \\
\vdots & & \vdots \\
0 & \ldots & \frac{1}{\sinh \left(l_{\beta_{n} / 2}\right)}
\end{array}\right)\left(\begin{array}{ccc}
\frac{\partial\left|\operatorname{tr} \rho \beta_{1}\right|}{\partial t_{\alpha_{1}}} & \ldots & \frac{\partial\left|\operatorname{tr} \rho \beta_{1}\right|}{\partial t_{\alpha_{n}}} \\
\vdots & & \vdots \\
\frac{\partial\left|\operatorname{tr} \rho \beta_{n}\right|}{\partial t_{\alpha_{1}}} & \ldots & \frac{\partial\left|\operatorname{tr} \rho \beta_{n}\right|}{\partial t_{\alpha_{n}}}
\end{array}\right) .
$$


We denote the second matrix in this product by $T=T(\mathcal{A}, \mathcal{B}, \rho)$. Since $\sinh \left(l_{\beta_{i}} / 2\right)$ is always strictly positive, we have the following immediate corollary of Proposition 4.6 which will be used to compute our examples of simplices of minima in the next section.

Corollary 4.7. Let $\mathcal{A}=\left\{\alpha_{1}, \ldots, \alpha_{n}\right\}, \mathcal{B}=\left\{\beta_{1}, \ldots, \beta_{n}\right\}$ be two pants decompositions of $S$, and let $\rho \in \operatorname{Teich}(S)$. Then

(a) If $\rho \in \overline{\mathcal{S}}_{\mathcal{A}, \mathcal{B}}$, then $\left.\operatorname{det} T\right|_{\rho}=0$.

(b) Suppose $\operatorname{rk}\left(\left.T\right|_{\rho}\right)=n-1$. Then $\rho \in \overline{\mathcal{S}}_{\mathcal{A}, \mathcal{B}}$ if and only if all entries in the adjoint matrix $\left.\operatorname{Adj} T\right|_{\rho}$ have the same sign (with possibly some entries vanishing).

4.2. Example 1: The simplex $\overline{\mathcal{S}}_{\alpha_{1}, \alpha_{2} ; \beta_{1}, \beta_{2}}$. We are now ready to compute our first explicit examples. Using Proposition 4.6 or Corollary 4.7, we shall find the simplex of minima for the twice-punctured torus $\overline{\mathcal{S}}_{\alpha_{1}, \alpha_{2} ; \beta_{1}, \beta_{2}}$, where $\alpha_{1}, \alpha_{2}, \beta_{1}, \beta_{2}$ are the curves given in Section 3. We express the results in terms of Fenchel-Nielsen $\alpha$-coordinates.

From Proposition 3.2 with $\theta_{1}=\theta_{2}=0$, we see that $\operatorname{tr} \rho \beta_{1}$ and $\operatorname{tr} \rho \beta_{2}$ are positive and given by

$$
\operatorname{tr} \rho \beta_{1}=-2\left\langle V_{1}\left(t_{1}\right), V_{2}\left(t_{2}\right)\right\rangle, \quad \operatorname{tr} \rho \beta_{2}=-2\left\langle V_{1}\left(t_{1}-l_{1}\right), V_{2}\left(t_{2}+l_{2}\right)\right\rangle .
$$

By computing derivatives (using the formulae in Remark 3 of Section 3.1), we have that the matrix $\left.T\right|_{\rho}$ of derivatives of traces is equal to

$$
T=\left(\begin{array}{ll}
-\left\langle e_{1}, V_{2}\right\rangle & -\left\langle V_{1}, e_{2}\right\rangle \\
-\left\langle e_{1}^{\prime}, V_{2}^{\prime}\right\rangle & -\left\langle V_{1}^{\prime}, e_{2}^{\prime}\right\rangle
\end{array}\right),
$$

where we have used the notation $V_{i}=V_{i}\left(t_{i}\right), e_{i}=e_{i}\left(t_{i}\right), V_{1}^{\prime}=V_{1}\left(t_{1}-l_{1}\right), e_{1}^{\prime}=$ $e_{1}\left(t_{1}-l_{1}\right), V_{2}^{\prime}=V_{2}\left(t_{2}+l_{2}\right), e_{2}^{\prime}=e_{2}\left(t_{2}+l_{2}\right)$. We denote by $T_{i j}$ the entries of $T$. The following lemma shows that some configurations of signs for the entries of $\left.T\right|_{\rho}$ are impossible.

Lemma 4.8. There is no $\rho$ in $\operatorname{Teich}(S)$ for which the entries of $\left.T\right|_{\rho}$ satisfy $T_{11} \leq 0$, $T_{12} \geq 0, T_{21} \geq 0, T_{22} \leq 0$. Moreover at most two entries of $T$ can be equal to zero.

Proof. Suppose that $T_{11} \leq 0$. If $t_{1}>0$, then, by looking at the Main Figure, $T_{12}=-\left\langle V_{1}, e_{2}\right\rangle<0$ (see Figure 5(a)); on the other hand, if $t_{1} \leq 0$, then $\left(t_{1}-l_{1}\right) / 2 \leq$ $-l_{1} / 2$, and this implies that $T_{21}=-\left\langle e_{1}^{\prime}, V_{2}^{\prime}\right\rangle<0$ (see Figure $5(\mathrm{~b})$ ). Therefore, the above configuration of signs cannot happen.

If $T_{11}=T_{12}=0$, then $t_{1}=t_{2}=0$. In this position, the two perpendiculars to the lines $L_{1}, L_{2}$ at $V_{1}^{\prime}, V_{2}^{\prime}$ respectively meet at $\infty$. Hence neither $T_{21}$ nor $T_{22}$ vanishes. Similarly, if $T_{21}=T_{22}=0$, then $T_{11} \neq 0, T_{12} \neq 0$. Suppose that three entries of $T$ are equal to zero. Then either $T_{11}=T_{12}=0$ or $T_{21}=T_{22}=0$, and we arrive at a contradiction.

Theorem 4.9. A point $\rho \in \operatorname{Teich}(S)$ is in $\overline{\mathcal{S}}_{\alpha_{1}, \alpha_{2} ; \beta_{1}, \beta_{2}}$ if and only if $\operatorname{det} T=0$ and $T_{11} \geq 0, T_{12} \leq 0, T_{21} \leq 0, T_{22} \geq 0$. The point $\rho$ is in $\mathcal{S}_{\alpha_{1}, \alpha_{2} ; \beta_{1}, \beta_{2}}$ if and only if no entry is equal to zero.

Proof. From Remark 1 of Section 3.1, the matrix $T$ is identically zero if and only if $t_{1}=t_{2}=l_{1}=l_{2}=0$, but this is impossible in Teich $(S)$. Hence, in $\operatorname{Teich}(S)$ the rank of $T$ is always greater than zero. By Corollary $4.7 \rho$ is in $\overline{\mathcal{S}}_{\alpha_{1}, \alpha_{2} ; \beta_{1}, \beta_{2}}$ if and 


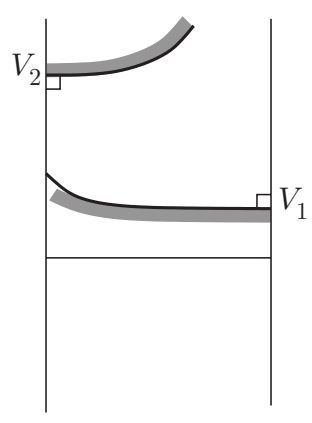

(a) $T_{11} \leq 0, t_{1}>0$

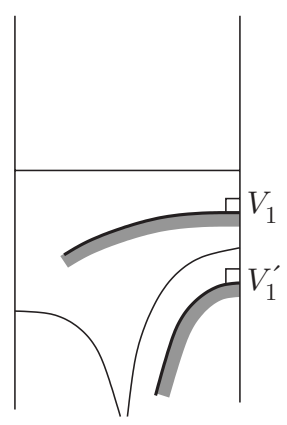

(b) $t_{1} \leq 0$

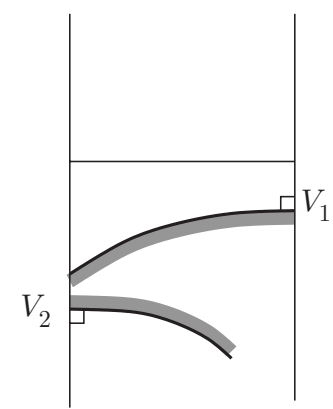

(c)

FIGURE 5 .

only if $\operatorname{rk} T=1$ and the configuration of signs for $T$ is

$$
\left(\begin{array}{ll}
+ & - \\
- & +
\end{array}\right) \text { or }\left(\begin{array}{ll}
- & + \\
+ & -
\end{array}\right) \text {. }
$$

By Lemma 4.8 the second configuration is impossible in Teich $(S)$.

The final statement follows since, as in Proposition [4.5] $\rho \in \mathcal{S}_{\alpha_{1}, \alpha_{2} ; \beta_{1}, \beta_{2}}$ if and only if the nullvectors are both strictly positive.

An alternative way of proving the first statement is by using Kerckhoff's derivative formula; we have

$$
\left(\frac{\partial l_{\beta_{i}}}{\partial t_{\alpha_{j}}}\right)=\left(\begin{array}{ll}
\cos \psi_{11} & \cos \psi_{12} \\
\cos \psi_{21} & \cos \psi_{22}
\end{array}\right),
$$

where $\psi_{i j}$ is the angle from $\beta_{i}$ to $\alpha_{j}$. Therefore, the second configuration of signs implies that the quadrilateral determined by the geodesics $\alpha_{1}, \alpha_{2}, \beta_{1}, \beta_{2}$ has all its angles greater than or equal to $\pi / 2$, which is impossible (this quadrilateral on the hyperbolic surface lifts to the quadrilateral of vertices $\left.V_{1}, V_{2}, V_{1}^{\prime}, V_{2}^{\prime}\right)$.

The following lemma contains further concrete information about the location of $\overline{\mathcal{S}}_{\alpha_{1}, \alpha_{2} ; \beta_{1}, \beta_{2}}$.

Lemma 4.10. If $\rho \in \overline{\mathcal{S}}_{\alpha_{1}, \alpha_{2} ; \beta_{1}, \beta_{2}}$, then $0 \leq t_{1} \leq l_{1}$ and $-l_{2} \leq t_{2} \leq 0$, $\left|\left\langle e_{1}, e_{2}\right\rangle\right|<1$ and $\left|\left\langle e_{1}^{\prime}, e_{2}^{\prime}\right\rangle\right|<1$.

Proof. Suppose $t_{1}<0$. By Theorem 4.9 $T_{11}=-\left\langle e_{1}, V_{2}\right\rangle \geq 0$, so the point $V_{2}$ must be as in Figure 5(c); but this implies $T_{12}=-\left\langle V_{1}, e_{2}\right\rangle>0$, which is a contradiction. Therefore, $t_{1} \geq 0$. Now $t_{1} \geq 0$, and $\left\langle e_{1}, V_{2}\right\rangle,\left\langle V_{1}, e_{2}\right\rangle$ have different signs; hence $t_{2} \leq 0$. Arguing similarly, $t_{1} \leq l_{1}$ and $-l_{2} \leq t_{2}$. Also, the fact that $T_{11}, T_{12}$ have different signs implies that $\left|\left\langle e_{1}, e_{2}\right\rangle\right|<1$, and $T_{21}, T_{22}$ having different signs implies that $\left|\left\langle e_{1}^{\prime}, e_{2}^{\prime}\right\rangle\right|<1$.

4.3. Subsimplices of $\overline{\mathcal{S}}_{\alpha_{1}, \alpha_{2} ; \beta_{1}, \beta_{2}}$. We now compute some subsimplices of $\overline{\mathcal{S}}_{\alpha_{1}, \alpha_{2} ; \beta_{1}, \beta_{2}}$, beginning with the boundary of $\overline{\mathcal{S}}_{\alpha_{1}, \alpha_{2} ; \beta_{1}, \beta_{2}}$, which consists of the union of the four subsimplices $\overline{\mathcal{S}}_{\alpha_{1} ; \beta_{1}, \beta_{2}}, \overline{\mathcal{S}}_{\alpha_{2} ; \beta_{1}, \beta_{2}}, \overline{\mathcal{S}}_{\alpha_{1}, \alpha_{2} ; \beta_{1}}, \overline{\mathcal{S}}_{\alpha_{1}, \alpha_{2} ; \beta_{2}}$. Set

$$
\Pi=\left\{\left(l_{1}, l_{2}, t_{1}, t_{2}\right) \mid t_{1}=t_{2}=0\right\} \subset \operatorname{Teich}(S) .
$$

This plane will come up repeatedly in what follows. 
Proposition 4.11. (a) The simplex $\overline{\mathcal{S}}_{\alpha_{1}, \alpha_{2} ; \beta_{1}}$ is the subset of Teich $(S)$ satisfying the conditions $T_{11}=T_{12}=0$, or, equivalently, $t_{1}=t_{2}=0$, the plane $\Pi$.

(b) The simplex $\overline{\mathcal{S}}_{\alpha_{1}, \alpha_{2} ; \beta_{2}}$ is the subset satisfying $T_{21}=T_{22}=0$, or, equivalently, $t_{1}=l_{1}, t_{2}=-l_{2}$.

(c) The simplex $\overline{\mathcal{S}}_{\alpha_{1} ; \beta_{1}, \beta_{2}}$ is the subset satisfying $T_{11}=T_{21}=0$, or, equivalently,

$$
\left\{\begin{array}{c}
\cosh d \tanh \frac{t_{1}}{2}+\tanh \frac{t_{2}}{2}=0, \\
\cosh d \tanh \frac{t_{1}-l_{1}}{2}+\tanh \frac{t_{2}+l_{2}}{2}=0 .
\end{array}\right.
$$

(d) The simplex $\overline{\mathcal{S}}_{\alpha_{2} ; \beta_{1}, \beta_{2}}$ is the subset satisfying $T_{12}=T_{22}=0$, or, equivalently,

$$
\left\{\begin{array}{c}
\cosh d \tanh \frac{t_{2}}{2}+\tanh \frac{t_{1}}{2}=0, \\
\cosh d \tanh \frac{t_{2}+l_{2}}{2}+\tanh \frac{t_{1}-l_{1}}{2}=0 .
\end{array}\right.
$$

Proof. Take $\rho \in \overline{\mathcal{S}}_{\alpha_{1}, \alpha_{2} ; \beta_{1}}$. Then, by Proposition 4.5, $(1,0)$ is a left nullvector of the matrix $M$, and also of $T$. This implies $T_{11}=T_{12}=0$, which is equivalent to $t_{1}=t_{2}=0$. Therefore $\overline{\mathcal{S}}_{\alpha_{1}, \alpha_{2} ; \beta_{1}}$ is contained in $\Pi$. In this case, we have $T_{21}<0$ and $T_{22}>0$ (by Lemma 4.8). Since the rank of $T$ is 1 , by Corollary 4.7 the whole plane $\Pi$ is contained in the simplex $\overline{\mathcal{S}}_{\alpha_{1}, \alpha_{2} ; \beta_{1}}$. We describe the other simplices similarly, just setting two entries of $T$ equal to zero and checking that the signs of the remaining entries are appropriate.

Remark. One can give an alternative proof of (c) and (d) using Fenchel-Nielsen $\beta$-coordinates. In these coordinates, consider the matrix $T^{\beta}=\left(\frac{\partial \operatorname{tr} \rho \alpha_{i}}{\partial t_{\beta_{j}}}\right)$. By Wolpert's antisymmetry formula, $T$ and $T^{\beta}$ are related by

$$
\left(\begin{array}{cc}
\frac{1}{\sinh \frac{l_{1}}{2}} & 0 \\
0 & \frac{1}{\sinh \frac{l_{\beta_{2}}}{2}}
\end{array}\right)\left(\begin{array}{cc}
T_{11} & T_{12} \\
T_{21} & T_{22}
\end{array}\right)=-\left(\begin{array}{cc}
T_{11}^{\beta} & T_{21}^{\beta} \\
T_{12}^{\beta} & T_{22}^{\beta}
\end{array}\right)\left(\begin{array}{cc}
\frac{1}{\sinh \frac{l_{\alpha_{1}}}{2}} & 0 \\
0 & \frac{1}{\sinh \frac{l_{\alpha_{2}}}{2}}
\end{array}\right)
$$

Then, by using the $\beta$-coordinates and arguing as in (a), the simplex $\overline{\mathcal{S}}_{\alpha_{1} ; \beta_{1}, \beta_{2}}$ is described by $T_{11}^{\beta}=T_{12}^{\beta}=0$. By the previous relation, this is equivalent to $T_{11}=$ $T_{21}=0$.

Next we compute $\overline{\mathcal{S}}_{\alpha_{1}+\alpha_{2} ; \beta_{1}, \beta_{2}}$ and $\overline{\mathcal{S}}_{\alpha_{1}, \alpha_{2} ; \beta_{1}+\beta_{2}}$, which are subsimplices of $\overline{\mathcal{S}}_{\alpha_{1}, \alpha_{2} ; \beta_{1}, \beta_{2}}$.

Proposition 4.12. (a) The simplex $\overline{\mathcal{S}}_{\alpha_{1}+\alpha_{2} ; \beta_{1}, \beta_{2}}$ is the subset of Teich $(S)$ described by the conditions $l_{1}=l_{2}, t_{1}=-t_{2}, 0 \leq t_{1} \leq l_{1}$.

(b) The simplex $\overline{\mathcal{S}}_{\alpha_{1}, \alpha_{2} ; \beta_{1}+\beta_{2}}$ is the subset of Teich $(S)$ described by the conditions

$$
t_{1}=l_{1} / 2, t_{2}=-l_{2} / 2, T_{11} \geq 0, T_{12} \leq 0, T_{21} \leq 0, T_{22} \geq 0 .
$$

In terms of Fenchel-Nielsen $\beta$-coordinates $\left(l_{\beta_{1}}, l_{\beta_{2}}, t_{\beta_{1}}, t_{\beta_{2}}\right)$, this simplex is described by the conditions $l_{\beta_{1}}=l_{\beta_{2}}, t_{\beta_{1}}=-t_{\beta_{2}}, 0 \leq-t_{\beta_{1}} \leq l_{\beta_{1}}$.

Proof. (a) By Propositions 4.5 and $4.6 \rho \in \overline{\mathcal{S}}_{\alpha_{1}+\alpha_{2} ; \beta_{1}, \beta_{2}}$ if and only if $(1,1)$ is a right nullvector of the matrix $M$ and the signs of this matrix are $\left({ }_{-}^{+}\right)$. By the relation between the matrices $M$ and $T,(1,1)$ is also a nullvector of $T$. Thus we must have

$$
\left\langle V_{1}, e_{2}\right\rangle+\left\langle e_{1}, V_{2}\right\rangle=0 \text { and }\left\langle V_{1}^{\prime}, e_{2}^{\prime}\right\rangle+\left\langle e_{1}^{\prime}, V_{2}^{\prime}\right\rangle=0 .
$$

Given fixed values of $t_{1}, l_{1}, l_{2}$, consider the function $g\left(t_{2}\right)=\left\langle V_{1}, e_{2}\right\rangle+\left\langle e_{1}, V_{2}\right\rangle$ of $t_{2}$. Using the formulae in Remark 3 of Section 3.1, its derivative is $g^{\prime}\left(t_{2}\right)=\frac{1}{2}\left\langle V_{1}, V_{2}\right\rangle+$ $\frac{1}{2}\left\langle e_{1}, e_{2}\right\rangle$, and is always negative. Indeed, $\left\langle V_{1}, V_{2}\right\rangle<0$ and $\left|\left\langle V_{1}, V_{2}\right\rangle\right|>\left\langle e_{1}, e_{2}\right\rangle$, 
because $\left|\left\langle e_{1}, e_{2}\right\rangle\right|$ either is less than 1 or is the hyperbolic cosine of the distance $d_{1}$ between two lines, whereas $\left|\left\langle V_{1}, V_{2}\right\rangle\right|$ is the hyperbolic cosine of the distance $d_{2}$ of two points in these lines, which, by their position, are never the ends of the common perpendicular segment. Therefore $d_{2}>d_{1}$. Then $g$ is strictly decreasing and takes the value 0 at most once. On the other hand, from the definitions $V_{1}=V_{1}\left(t_{1}\right)$ and $V_{2}=V_{2}\left(t_{2}\right)$, it is clear that $g\left(t_{2}\right)=0$ when $t_{2}=-t_{1}$. Therefore, $\left\langle V_{1}, e_{2}\right\rangle+\left\langle e_{1}, V_{2}\right\rangle=0$ if and only if $t_{2}=-t_{1}$.

Recalling the definitions $V_{1}^{\prime}=V_{1}\left(t_{1}-l_{1}\right), V_{2}^{\prime}=V_{2}\left(t_{2}+l_{2}\right)$ and so on, the same argument shows that $\left\langle V_{1}^{\prime}, e_{2}^{\prime}\right\rangle=-\left\langle e_{1}^{\prime}, V_{2}^{\prime}\right\rangle$ if and only if $t_{2}+l_{2}=-\left(t_{1}-l_{1}\right)$. Then, we obtain $l_{1}=l_{2}$. Finally, the matrix has the desired signs if and only if $0 \leq t_{1} \leq l_{1}$.

(b) A similar proof gives the description of the simplex $\overline{\mathcal{S}}_{\alpha_{1}, \alpha_{2} ; \beta_{1}+\beta_{2}}$ in terms of the Fenchel-Nielsen $\beta$-coordinates, from which one easily gets the description in terms of the $\alpha$-coordinates.

The line of minima $\mathcal{L}_{\alpha_{1}+\alpha_{2} ; \beta_{1}+\beta_{2}}$ is clearly the intersection of the two above simplices. Therefore we have

Corollary 4.13. The line of minima $\mathcal{L}_{\alpha_{1}+\alpha_{2} ; \beta_{1}+\beta_{2}}$ is the subset of Teich $(S)$ given by $l_{1}=l_{2}, t_{1}=-t_{2}, t_{1}=l_{1} / 2$. A point $\rho \in \operatorname{Teich}(S)$ is in $\mathcal{L}_{\alpha_{1}+\alpha_{2} ; \beta_{1}+\beta_{2}}$ if and only if $\psi_{11}=\psi_{22}=\pi-\psi_{12}=\pi-\psi_{21}$, where $\psi_{i j}$ is the angle from the geodesic representing $\beta_{i}$ to the geodesic representing $\alpha_{j}$.

Proof. By Proposition 4.5 $\rho \in \mathcal{L}_{\alpha_{1}+\alpha_{2} ; \beta_{1}+\beta_{2}}$ if and only if $(1,1)$ is a left and right nullvector of the matrix $M$ of derivatives of the lengths

$$
\left(\begin{array}{cc}
\cos \psi_{11} & \cos \psi_{12} \\
\cos \psi_{21} & \cos \psi_{22}
\end{array}\right)
$$

Therefore, we must have $\cos \psi_{11}=\cos \psi_{22}=-\cos \psi_{12}=-\cos \psi_{21}$, and the quadrilateral of vertices $V_{1}, V_{2}, V_{1}^{\prime}, V_{2}^{\prime}$ has all its angles equal to $\psi_{11}$.

4.4. Example 2: The simplex $\overline{\mathcal{S}}_{\alpha_{1}, \alpha_{2} ; \beta_{1}, \beta_{1}}$. Next we compute the simplex $\overline{\mathcal{S}}_{\alpha_{1}, \alpha_{2} ; \beta_{1}, \delta_{1}}$. This turns out to be an example in which the map $\Phi$ of Section 4.1 is not injective. In fact we shall show that (for any fixed pair of values for $l_{\alpha_{1}}, l_{\alpha_{2}}$ ), each of the three simplices $\overline{\mathcal{S}}_{\alpha_{1}, \alpha_{2} ; \beta_{1}, \delta_{1}}, \overline{\mathcal{S}}_{\alpha_{1}, \alpha_{2} ; \beta_{1}, \delta_{2}}$ (whose computation is completely analogous) and $\overline{\mathcal{S}}_{\alpha_{1}, \alpha_{2} ; \beta_{1}}$ (which we already computed in Proposition 4.11) coincides exactly with the plane $\Pi:=\left\{t_{1}=t_{2}=0\right\}$. Thus a point in $\Pi$ will lie simultaneously on the lines of minima for many distinct pairs of laminations $\mu, \nu$. By Proposition 4.3 a point lies on $\mathcal{L}_{\mu, \nu}$ if and only if some convex combination of $\frac{\partial}{\partial t_{\mu}}$ and $\frac{\partial}{\partial t_{\nu}}$ vanishes, in other words, if and only if $\frac{\partial}{\partial t_{\mu}}$ and $\frac{\partial}{\partial t_{\nu}}$ point in opposite directions in the tangent space at $p$. Figure 6 below gives a complete picture of the tangent space to $\Pi$, from which one can read off exactly which vectors are paired to which in this way.

We begin by computing the trace of $\rho \delta_{1}$.

Lemma 4.14. Let $\rho=\left(l_{1}, l_{2}, t_{1}, t_{2}\right) \in \operatorname{Teich}(S)$. Then

$$
\operatorname{tr} \rho \delta_{1}=-4 \sinh ^{2}\left(l_{2} / 2\right) \sinh ^{2} d \cosh ^{2}\left(t_{1} / 2\right)+2 .
$$

Proof. From the formula for the trace of a commutator, we have

$$
\operatorname{tr} \rho \delta_{1}=\left(\operatorname{tr} \rho \beta_{1}\right)^{2}+\left(\operatorname{tr} \rho \alpha_{2}\right)^{2}+\left(\operatorname{tr} \rho \beta_{1} \alpha_{2}\right)^{2}-\operatorname{tr} \rho \beta_{1} \operatorname{tr} \rho \alpha_{2} \operatorname{tr} \rho \beta_{1} \alpha_{2}-2 .
$$


We also know that

$$
\begin{aligned}
\operatorname{tr} \rho \alpha_{2} & =2 \cosh \left(l_{2} / 2\right), \\
\operatorname{tr} \rho \beta_{1} & =\operatorname{tr}\left(T_{L_{1}}\left(t_{1}\right) T_{M}(d) T_{L_{1}}\left(t_{2}\right) T_{M}(d)\right)=-2\left\langle V_{1}\left(t_{1}\right), V_{2}\left(t_{2}\right)\right\rangle, \\
\operatorname{tr} \rho \beta_{1} \alpha_{2} & =\operatorname{tr}\left(T_{L_{1}}\left(t_{1}\right) T_{M}(d) T_{L_{1}}\left(t_{2}+l_{2}\right) T_{M}(d)\right)=-2\left\langle V_{1}\left(t_{1}\right), V_{2}\left(l_{2}+t_{2}\right)\right\rangle .
\end{aligned}
$$

As usual, we write $V_{1}=V_{1}\left(t_{1}\right), V_{2}^{\prime}=V_{2}\left(l_{2}+t_{2}\right)$, and so on. Then

$$
\operatorname{tr} \rho \delta_{1}=4 \cosh ^{2}\left(l_{2} / 2\right)+4\left\langle V_{1}, V_{2}\right\rangle^{2}+4\left\langle V_{1}, V_{2}^{\prime}\right\rangle^{2}-8\left\langle V_{1}, V_{2}\right\rangle\left\langle V_{1}, V_{2}^{\prime}\right\rangle \cosh \left(l_{2} / 2\right)-2 \text {. }
$$

Expanding $\left\langle V_{1}, V_{2}^{\prime}\right\rangle$, we get

$$
\left\langle V_{1}, V_{2}^{\prime}\right\rangle=\left\langle V_{1}, V_{2}\right\rangle \cosh \left(l_{2} / 2\right)+\left\langle V_{1}, e_{2}\right\rangle \sinh \left(l_{2} / 2\right)
$$

so that

$$
\operatorname{tr} \rho \delta_{1}=4 \cosh ^{2}\left(l_{2} / 2\right)+4 \sinh ^{2}\left(l_{2} / 2\right)\left(-\left\langle V_{1}, V_{2}\right\rangle^{2}+\left\langle V_{1}, e_{2}\right\rangle^{2}\right)-2 .
$$

By direct computation we get

$$
-\left\langle V_{1}, V_{2}\right\rangle^{2}+\left\langle V_{1}, e_{2}\right\rangle^{2}=-1-\sinh ^{2} d \cosh ^{2}\left(t_{1} / 2\right),
$$

finally giving the formula for $\operatorname{tr} \rho \delta_{1}$.

Theorem 4.15. The simplex $\mathcal{S}_{\alpha_{1}, \alpha_{2} ; \beta_{1}, \delta_{1}}$, and its faces $\mathcal{S}_{\alpha_{1}, \alpha_{2} ; \beta_{1}}$ and $\mathcal{S}_{\alpha_{1} ; \beta_{1}, \delta_{1}}$, are each equal to the plane $\Pi$.

Proof. We already know that the simplex $\overline{\mathcal{S}}_{\alpha_{1}, \alpha_{2} ; \beta_{1}}$ is equal to $\Pi$ by Proposition 4.11 (a). We compute the matrix $\left.T\right|_{\rho}$ of the derivatives of the traces of $\rho \beta_{1}, \rho \delta_{1}$ with respect to $t_{i}$. Since $\delta_{1}$ is a commutator, its trace is negative, and so, by Lemma 4.14 $\left|\operatorname{tr} \rho \delta_{1}\right|=4 \sinh ^{2} \frac{l_{2}}{2} \sinh ^{2} d \cosh ^{2} \frac{t_{1}}{2}-2$. Then we get

$$
\left.T\right|_{\rho}=\left(\begin{array}{cc}
-\left\langle e_{1}\left(t_{1}\right), V_{2}\left(t_{2}\right)\right\rangle & -\left\langle V_{1}\left(t_{1}\right), e_{2}\left(t_{2}\right)\right\rangle \\
2 \sinh ^{2}\left(l_{2} / 2\right) \sinh ^{2} d \sinh t_{1} & 0
\end{array}\right) .
$$

If $\rho \in \overline{\mathcal{S}}_{\alpha_{1}, \alpha_{2} ; \beta_{1}, \delta_{1}}$ then, by Corollary 4.7 we have $\left.\operatorname{det} T\right|_{\rho}=0$, so that either $t_{1}=0$ or $\left\langle V_{1}, e_{2}\right\rangle=0$. If $t_{1}=0$ and $\left\langle V_{1}, e_{2}\right\rangle \neq 0$, then $-\left\langle e_{1}, V_{2}\right\rangle$ and $-\left\langle V_{1}, e_{2}\right\rangle$ have the same sign, which is impossible by Corollary 4.7 (b). If $\left\langle V_{1}, e_{2}\right\rangle=0$ and $t_{1} \neq 0$, then $t_{1}$ and $-\left\langle e_{1}, V_{2}\right\rangle$ have the same sign, which again is impossible. Therefore we must have $t_{1}=0$ and $\left\langle V_{1}, e_{2}\right\rangle=0$, which is equivalent to $t_{1}=0, t_{2}=0$. Hence $\overline{\mathcal{S}}_{\alpha_{1}, \alpha_{2} ; \beta_{1}, \delta_{1}}$ and $\overline{\mathcal{S}}_{\alpha_{1} ; \beta_{1}, \delta_{1}}$ are contained in $\Pi$. Note that the matrix $T$ is then identically zero.

Let us prove now that $\Pi \subset \overline{\mathcal{S}}_{\alpha_{1} ; \beta_{1}, \delta_{1}}$. At a point $\rho \in \Pi$ the matrices $T$ and $M$ are identically zero. Hence, when expressing the tangent vectors $\left.\frac{\partial}{\partial t_{\alpha_{1}}}\right|_{\rho}$ and $\left.\frac{\partial}{\partial t_{\alpha_{2}}}\right|_{\rho}$ in terms of the coordinates induced by Fenchel-Nielsen coordinates with respect to $\left\{\beta_{1}, \delta_{1}\right\}$, we obtain

$$
\left(\begin{array}{c}
\frac{\partial}{\partial t_{\alpha_{1}}} \\
\frac{\partial}{\partial t_{\alpha_{2}}}
\end{array}\right)=\left(\begin{array}{ll}
\frac{\partial t_{\beta_{1}}}{\partial t_{\alpha_{1}}} & \frac{\partial t_{\delta_{1}}}{\partial t_{\alpha_{1}}} \\
\frac{\partial t_{\beta_{1}}}{\partial t_{\alpha_{2}}} & \frac{\partial t_{\delta_{1}}}{\partial t_{\alpha_{2}}}
\end{array}\right)\left(\begin{array}{c}
\frac{\partial}{\partial t_{\beta_{1}}} \\
\frac{\partial}{\partial t_{\delta_{1}}} .
\end{array}\right) .
$$

Denote the matrix in this formula by $N=\left(\begin{array}{ll}a & b \\ c & d\end{array}\right)$. As seen in the proof of Proposition 4.6 its associated linear map is injective on the nullspace of $M$, which in this case is 2-dimensional. Therefore $N$ is invertible. We shall show that $a$ and $b$ have the same sign, so that $\frac{\partial}{\partial t_{\alpha_{1}}}$ is proportional to a positive linear combination of $\frac{\partial}{\partial t_{\beta_{1}}}$ and $\frac{\partial}{\partial t_{\delta_{2}}}$. By Proposition 4.3 it will follow that $\rho$ is in the line of minima $\mathcal{L}_{\alpha_{1} ; a \beta_{1}+b \delta_{1}}$, and hence in $\overline{\mathcal{S}}_{\alpha_{1} ; \beta_{1}, \delta_{1}}$. 
Since $\alpha_{2}, \beta_{1}, \delta_{1}$ do not fill up the surface, $c$ and $d$ have opposite signs: otherwise we would have a contradiction to Proposition 4.3. Inverting $N$, we find that

$$
\left(\begin{array}{l}
\frac{\partial}{\partial t_{\beta_{1}}} \\
\frac{\partial}{\partial t_{\delta_{1}}}
\end{array}\right)=\left(\begin{array}{ll}
\frac{d}{\operatorname{det} N} & \frac{-b}{\operatorname{det} N} \\
\frac{-c}{\operatorname{det} N} & \frac{a}{\operatorname{det} N}
\end{array}\right)\left(\begin{array}{c}
\frac{\partial}{\partial t_{\alpha_{1}}} \\
\frac{\partial}{\partial t_{\alpha_{2}}}
\end{array}\right)
$$

Since $\alpha_{1}, \alpha_{2}, \delta_{1}$ do not fill up the surface, we deduce that $-c$ and $a$ have opposite signs. Since the simplex $\overline{\mathcal{S}}_{\alpha_{1}, \alpha_{2} ; \beta_{1}}$ is equal to $\Pi$, we know that $\left.\frac{\partial}{\partial t_{\beta_{1}}}\right|_{\rho}$ is a negative linear combination of $\left.\frac{\partial}{\partial t_{\alpha_{1}}}\right|_{\rho}$ and $\left.\frac{\partial}{\partial t_{\alpha_{2}}}\right|_{\rho}$. Therefore, $d$ and $-b$ have the same sign. Combining all this, we deduce that $a$ and $b$ have the same sign.

Finally we prove that $\Pi \subset \mathcal{S}_{\alpha_{1}, \alpha_{2} ; \beta_{1}, \delta_{1}}$. Since $\Pi=\overline{\mathcal{S}}_{\alpha_{1}, \alpha_{2} ; \beta_{1}}=\overline{\mathcal{S}}_{\alpha_{1} ; \beta_{1} ; \delta_{1}}$, at a point $\rho \in \Pi$ there exist positive numbers $a^{\prime}, b^{\prime}, c^{\prime}, d^{\prime}$ so that

$$
\frac{\partial}{\partial t_{\beta_{1}}}=-a^{\prime} \frac{\partial}{\partial t_{\alpha_{1}}}-b^{\prime} \frac{\partial}{\partial t_{\alpha_{2}}} \text { and } c^{\prime} \frac{\partial}{\partial t_{\beta_{1}}}+d^{\prime} \frac{\partial}{\partial t_{\delta_{1}}}=-\frac{\partial}{\partial t_{\alpha_{1}}}
$$

Adding these equations we get a strictly positive linear combination of $\frac{\partial}{\partial t_{\beta_{1}}}$ and $\frac{\partial}{\partial t_{\delta_{1}}}$ equal to a negative linear combination of $\frac{\partial}{\partial t_{\alpha_{1}}}$ and $\frac{\partial}{\partial t_{\alpha_{2}}}$. Again, by Proposition 4.3 we get that $\rho \in \mathcal{S}_{\alpha_{1}, \alpha_{2} ; \beta_{1}, \delta_{1}}$.

The remaining possible combinations of this family of curves are easily dealt with. Since the triples of curves $\alpha_{2}, \beta_{1}, \delta_{1}$ and $\alpha_{1}, \alpha_{2}, \delta_{1}$ do not fill up the surface, they do not define any simplex of minima in Teich $(S)$. In analogy to Theorem 4.15 one proves that the simplex $\overline{\mathcal{S}}_{\alpha_{1}, \alpha_{2} ; \beta_{1}, \delta_{2}}$ equals $\Pi$. All the pairings of tangent vectors obtained for these various simplices of minima have been collected in Figure 6, which shows the subspace of $T_{\rho}($ Teich $(S))$ generated by $\frac{\partial}{\partial t_{\alpha_{1}}}, \frac{\partial}{\partial t_{\alpha_{2}}}$ at a point $\rho \in \Pi$.

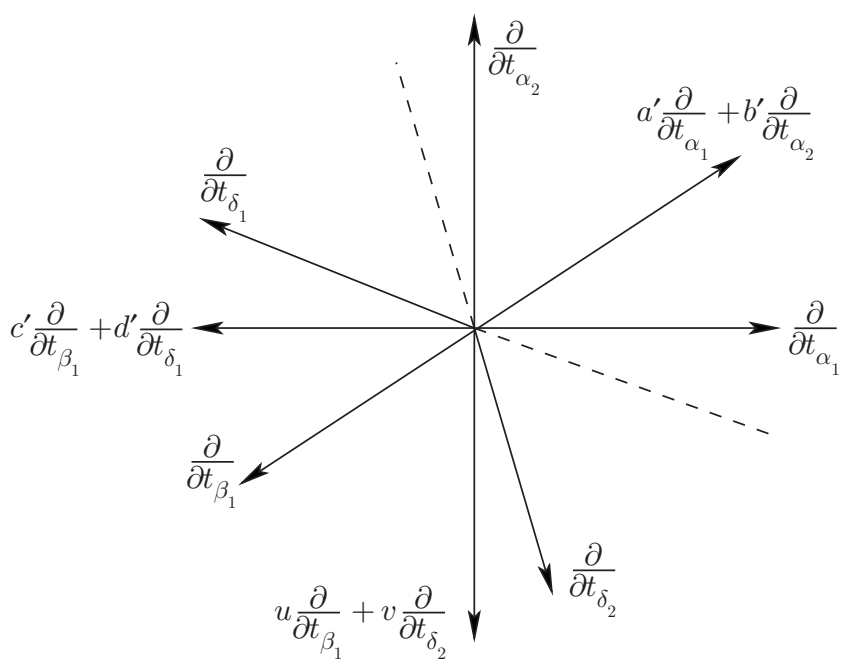

Figure 6. 


\section{EXAMPLES OF PLEATING VARIETIES}

In this section we calculate the pleating varieties corresponding to the simplices in the last section. Once again, we begin by recalling some general results. Throughout, we make the natural identification of $\operatorname{Teich}(S)$ with Fuchsian space $\mathcal{F} \subset \mathcal{Q F}$.

5.1. General results. The first result concerns how pleating varieties meet Fuchsian space. In [20], the second author proved the following:

Theorem 5.1 (20, Theorem 5.1). Suppose that pants decompositions $\mathcal{A}, \mathcal{B}$ fill up $S$. Then $\mathcal{F} \cap \overline{\mathcal{P}_{\mathcal{A}, \mathcal{B}}} \subset \overline{\mathcal{S}}_{\mathcal{A}, \mathcal{B}}$, and $\mathcal{F} \cap \overline{\mathcal{P}_{\mu, \nu}} \subset \overline{\mathcal{L}}_{\mu, \nu}$, where $\mu, \nu$ are measured laminations which are combinations of the curves in $\mathcal{A}$ and $\mathcal{B}$, respectively.

From the proof of this result we extract the following lemma, which will be used to prove Theorems 5.7 and 5.21 The angles $\theta_{\alpha_{i}}$ (resp. $\theta_{\beta_{i}}$ ) are the imaginary parts of the twist parameters in the Fenchel-Nielsen coordinates relative to the pants decompositions $\mathcal{A}$ (resp. $\mathcal{B})$.

Lemma 5.2. Let $\sigma:[0,1] \rightarrow \mathcal{Q F}(S)$ be a path such that $\sigma(0)=p \in \mathcal{F}, \sigma(t) \notin \mathcal{F}$ for $t \neq 0$, and $\lambda_{\alpha_{i}}(\sigma(t)), \lambda_{\beta_{i}}(\sigma(t)) \in \mathbb{R}$. Suppose that for all $i$ the limit, as $t \rightarrow 0$, of $\frac{\theta_{\alpha_{i}}}{\sum_{j}\left|\theta_{\alpha_{j}}\right|}(\sigma(t))$ exists, denoted by $a_{i}$. Then $\left(a_{1}, \ldots, a_{n}\right)^{T}$ is a right nullvector of $M(\mathcal{A}, \mathcal{B}, p)$. Further, the limit $b_{i}$ of $\theta_{\beta_{i}} / \sum_{j}\left|\theta_{\beta_{j}}\right|$ and the limit $k$ of $\sum_{i}\left|\theta_{\beta_{i}}\right| / \sum_{i}\left|\theta_{\alpha_{i}}\right|$ also exist, and we have

$$
k\left(b_{1}, \ldots, b_{n}\right)=\left(\left(\left.\frac{\partial t_{\beta_{i}}}{\partial t_{\alpha_{j}}}\right|_{p}\right)\left(\begin{array}{c}
a_{1} \\
\vdots \\
a_{n}
\end{array}\right)\right)^{T} \text { and }\left.\sum_{i} a_{i} \frac{\partial}{\partial t_{\alpha_{i}}}\right|_{p}=\left.k \sum_{i} b_{i} \frac{\partial}{\partial t_{\beta_{i}}}\right|_{p} .
$$

Remark. (1) In Theorem 5.1 in [20, we allowed the bending laminations to vary in $\mathcal{P}_{\mathcal{A}, \mathcal{B}}$ and extracted subsequences along which the limits $\left(a_{1}, \ldots, a_{n}\right),\left(b_{1}, \ldots, b_{n}\right)$ existed. However, once we assume the existence of the projective limits for the angles $\theta_{\alpha_{i}}$, the limit for the angles $\theta_{\beta_{i}}$ is automatic since, as proved in [20], it satisfies the matrix equation in the above statement.

(2) We have to be careful about angles when we change from one set of pants curves $\mathcal{A}$ to another set $\mathcal{A}^{\prime}$ in which some but not all of the pants curves are different. In this situation, even if a curve $\alpha$ is contained in both sets $\mathcal{A}$ and $\mathcal{A}^{\prime}$, the angle $\theta_{\alpha}$ relative to $\mathcal{A}$ is not in general equal to the angle $\theta_{\alpha}$ relative to $\mathcal{A}^{\prime}$. This point will arise in Example 2 below.

(3) If the path $\sigma$ in Lemma 5.2 is contained in the pleating variety $\mathcal{P}_{\mathcal{A}, \mathcal{B}}$, then all the $\theta_{\alpha_{i}}$ have the same sign and $\theta_{\alpha_{i}} / \theta_{\beta_{j}}<0$. Therefore, if $a_{i} \geq 0$, then $b_{i} \leq 0$. If we consider the measured laminations $\mu=\sum a_{i} \alpha_{i}$ and $\nu=\sum\left(-b_{i}\right) \beta_{i}$, then the last claim of the lemma gives $\frac{\partial}{\partial t_{\mu}}=-k \frac{\partial}{\partial t_{\nu}}$, with $k>0$, the sign of $k$ agreeing with Proposition 4.3 .

It was also conjectured (20], Conjecture 6.5) that for arbitrary laminations $\mu, \nu$, the closure of the pleating variety $\mathcal{P}_{\mu, \nu}$ meets $\mathcal{F}$ exactly in the simplex $\mathcal{S}_{\mu, \nu}, 1$ For rational laminations this conjecture reduces to:

Conjecture 5.3. Let $p$ be in the simplex of minima of $\overline{\mathcal{S}}_{\mathcal{A}, \mathcal{B}}$. Then $p$ is in the closure of $\mathcal{P}_{\mathcal{A}, \mathcal{B}}$.

\footnotetext{
${ }^{1}$ Added in proof: This conjecture has now been proved; see [21].
} 
The conjecture was proved $([20]$, Theorem 6.4) in the case in which $p$ is in the interior of the simplex and $\operatorname{rk} M(\mathcal{A}, \mathcal{B}, p)=n-1$. However, as we saw in the last section, the case $\operatorname{rk} M(\mathcal{A}, \mathcal{B}, p)<n-1$ may occur. The obstacle to proving the conjecture in general was to demonstrate the existence of a path starting at $p$ and moving out of $\mathcal{F}$ into $\mathcal{Q F}$ along which all lengths stay real and all angles have the same sign. In fact, assuming such a path, we can apply the following result, proved in [11] for the once punctured torus (Theorems 6.1 and 7.2), which extends with only trivial changes to higher genus; see also [6].

Theorem 5.4. Suppose that $p$ is in the simplex of minima $\mathcal{S}_{\mathcal{A}, \mathcal{B}}$ and that there is a path $\sigma:[0,1] \rightarrow \mathcal{Q F}$ such that $\sigma(0)=p$ and $\sigma(t) \notin \mathcal{F}$ for $t>0$. Suppose also that for $t>0$ we have $\lambda_{\alpha}(\sigma(t)), \lambda_{\beta}(\sigma(t)) \in \mathbb{R}$, and that the angles $\theta_{\alpha}(\sigma(t))$ all have the same sign and likewise the angles $\theta_{\beta}(\sigma(t))$ all have the same sign (possibly zero). Then there exists $\epsilon>0$ such that $\sigma((0, \epsilon)) \subset \mathcal{P}_{\mathcal{A}, \mathcal{B}}$.

To determine the pleating variety completely, we need to extend the path away from a neighbourhood of Fuchsian space. For this, ones needs the local pleating theorem ([12], Theorem 8.1) and the limit pleating theorem ([12], Theorem 5.1). The proofs of both these closely related results are much easier in the rational case (see [13], Theorem 3.7, and [16], Theorem 4.8), and extend without difficulty from once punctured tori to the general case. (The idea is to link each flat plane in $\partial \mathcal{C}$ to a disk in the regular set whose boundary contains the limit set of the Fuchsian subgroup which stabilises the plane in question. Such disks are called peripheral. One then has to prove that, provided no bending angle goes to 0 or $\pi$, peripheral disks persist under small deformations keeping traces real, and under taking limits. When an angle tends to $\pi$, disks become tangent and a group element becomes parabolic. When an angle tends to 0, two or more distinct peripheral disks may merge.)

Theorem 5.5 (Local pleating theorem). Suppose that $\rho$ is in the pleating variety $\mathcal{P}_{\mathcal{A}, \mathcal{B}}$. Suppose that there is a path $\sigma:[0, \epsilon) \rightarrow \mathcal{R}(S)$ such that $\sigma(0)=\rho$, $\lambda_{\alpha}(\sigma(t)), \lambda_{\beta}(\sigma(t)) \in \mathbb{R}-\{0\}$, and such that the angles $\theta_{\alpha}(\sigma(t)), \theta_{\beta}(\sigma(t)) \neq 0, \pi$. Then there exists $0<\epsilon^{\prime} \leq \epsilon$ such that $\sigma\left(\left(0, \epsilon^{\prime}\right)\right) \subset \mathcal{P}_{\mathcal{A}, \mathcal{B}}$.

Theorem 5.6 (Limit pleating theorem). Suppose that $\sigma:[0,1] \rightarrow \mathcal{R}(S)$ is a path such that $\sigma([0,1)) \subset \mathcal{P}_{\mathcal{A}, \mathcal{B}}, \lambda_{\alpha}(\sigma(1)), \lambda_{\beta}(\sigma(1)) \in \mathbb{R}^{+}$and $\theta_{\alpha}(\sigma(1)), \theta_{\beta}(\sigma(1)) \neq 0, \pi$. Then $\sigma(1) \in \mathcal{P}_{\mathcal{A}, \mathcal{B}}$.

Theorem 5.6 is also proved in [1].

Taken together, these three theorems show that, moving along a path starting in either $\mathcal{S}_{\mathcal{A}, \mathcal{B}}$ or $\mathcal{P}_{\mathcal{A}, \mathcal{B}}$ and keeping all traces real and all angles with the correct sign, we remain in $\mathcal{P}_{\mathcal{A}, \mathcal{B}}$ until either some bending angle becomes 0 or $\pm \pi$ or some length becomes zero. The upshot is a purely analytic condition for determining whether certain points are in $\mathcal{Q F}$, allowing one to determine the boundary of $\mathcal{P}_{\mathcal{A}, \mathcal{B}}$ without the need for any further checks on the behaviour of any other traces.

In the examples we calculate, we shall be able to explicitly find paths with the properties in the above results, circumventing the problem in the proof of Conjecture 5.3 above. These special paths will not only give us a complete proof of the conjecture in this case, but also allow us to completely locate the pleating varieties in question together with their boundaries.

These paths, which we call $\alpha$-lines, correspond to pure bends away from points starting in the relevant simplex of minima in $\mathcal{F}$. Along an $\alpha$-line, the lengths $l_{\alpha_{i}}$ are 
fixed, as is the ratio $\tan \left(\theta_{\alpha_{1}} / 2\right): \tan \left(\theta_{\alpha_{2}} / 2\right)$. This ratio is chosen in such a way that the traces of the other pair of curves in question $\left(\beta_{1}, \beta_{2}\right.$ or $\left.\beta_{1}, \delta_{1}\right)$ automatically stay real. In our first example $\mathcal{P}_{\alpha_{1}, \alpha_{2} ; \beta_{1}, \beta_{2}}$, we have seen that the corresponding simplex is embedded in $\mathcal{F}$ and there is exactly one $\alpha$-line leaving from each point in $\mathcal{S}_{\alpha_{1}, \alpha_{2} ; \beta_{1}, \beta_{2}}$.

In our second example $\mathcal{P}_{\alpha_{1}, \alpha_{2} ; \beta_{1}, \delta_{1}}$, we are in the degenerate case in which $\operatorname{rk} M=$ $0<n-1$ and the simplex is not embedded. In this case there will be many $\alpha$-lines meeting $\mathcal{F}$ in a single point of the simplex, one for each possible value of the angle ratio. Along these paths, all of the traces of $\alpha_{1}, \alpha_{2}, \beta_{1}, \delta_{1}, \delta_{2}$ stay real.

Now, as described on page 625 , if disjoint curves $\gamma, \gamma^{\prime}$ have real trace (thus, a pants decomposition of the twice punctured torus), there exists an invariant pleated surface $\mathrm{Pl}_{\gamma, \gamma^{\prime}}$, made by gluing the flat hyperbolic pants with boundary curves $\gamma, \gamma^{\prime}$. The bending angles of this surface are the angles between the two planes. Such a surface is a component of $\partial \mathcal{C}$ if and only if it is convex and embedded. Thus to determine when a curve is in the pleating locus, we have only to look at the signs of relevant angles. Using our analysis of lines of minima, we shall be able to do this along each $\alpha$-line and thus give a complete description of how the several distinct pleating varieties corresponding to these various curves meet each other and meet $\mathcal{F}$. The final picture, Figure 9, mirrors the infinitesimal picture in the tangent space, Figure 6 .

Non-singularity. A crucial feature of the results in 12 for the once punctured torus was that the pleating varieties $\mathcal{P}_{\gamma, \delta}$ for simple closed curves $\gamma, \delta$ were non-singular in the sense that the restriction of the map $q \mapsto\left(\lambda_{\gamma}(q), \lambda_{\delta}(q)\right)$ is a holomorphic bijection in a neighbourhood of $\mathcal{P}_{\gamma, \delta}$, and hence the restriction of this map to $\mathcal{P}_{\gamma, \delta}$ is a diffeomorphism onto its image in $\mathbb{R}^{+} \times \mathbb{R}^{+}$. We shall show that in our examples, the analogous map

$$
q \mapsto\left(\lambda_{\alpha_{1}}(q), \lambda_{\alpha_{2}}(q), \lambda_{\beta_{1}}(q), \lambda_{\beta_{2}}(q)\right)
$$

is a holomorphic bijection in a neighbourhood of $\mathcal{P}_{\mathcal{A}, \mathcal{B}}$.

5.2. Example 1: The pleating variety $\mathcal{P}_{\alpha_{1}, \alpha_{2} ; \beta_{1}, \beta_{2}}$. We shall compute the pleating variety

$$
\mathcal{P}_{\alpha_{1}, \alpha_{2} ; \beta_{1}, \beta_{2}}=\left\{\rho \in \mathcal{Q F}:\left|\mathrm{pl}^{+}\right|=\alpha_{1} \cup \alpha_{2},\left|\mathrm{pl}^{-}\right|=\beta_{1} \cup \beta_{2}\right\}
$$

in terms of the complex Fenchel-Nielsen $\alpha$-coordinates $\left(\lambda_{1}, \lambda_{2}, \tau_{1}, \tau_{2}\right)=\left(l_{1}+i \varphi_{1}\right.$, $\left.l_{2}+i \varphi_{2}, t_{1}+i \theta_{1}, t_{2}+i \theta_{2}\right)$. The description involves the matrix

$$
T=\left(\begin{array}{ll}
-\left\langle e_{1}, V_{2}\right\rangle & -\left\langle V_{1}, e_{2}\right\rangle \\
-\left\langle e_{1}^{\prime}, V_{2}^{\prime}\right\rangle & -\left\langle V_{1}^{\prime}, e_{2}^{\prime}\right\rangle
\end{array}\right)
$$

from Section 4.2 The following theorem gives a complete description of $\mathcal{P}_{\alpha_{1}, \alpha_{2} ; \beta_{1}, \beta_{2}}$, showing that it is actually just the product of an open interval with the simplex of minima $\mathcal{S}_{\alpha_{1}, \alpha_{2} ; \beta_{1}, \beta_{2}}$. To see this, notice that equations (5) and (6i) are exactly the equations of $\mathcal{S}_{\alpha_{1}, \alpha_{2} ; \beta_{1}, \beta_{2}}$ (Theorem 4.9). From each point of $\mathcal{S}_{\alpha_{1}, \alpha_{2} ; \beta_{1}, \beta_{2}}$ there emanates a unique $\alpha$-line given by (7), which continues until an endpoint which is the first point at which (8) fails.

The theorem will be proved using two auxiliary propositions (5.9) and 5.11) which are stated and proved later in the section. 
Theorem 5.7. The pleating variety $\mathcal{P}_{\alpha_{1}, \alpha_{2} ; \beta_{1}, \beta_{2}}$ is the subset of points $\left(l_{1}+i \varphi_{1}\right.$, $\left.l_{2}+i \varphi_{2}, t_{1}+i \theta_{1}, t_{2}+i \theta_{2}\right) \in \mathbb{C}^{4}$ satisfying

$$
\begin{aligned}
& \varphi_{1}=\varphi_{2}=0, \\
& 0<\theta_{1}, \theta_{2}<\pi \\
& \operatorname{det} T=0 \text {, } \\
& T_{11}>0, T_{12}<0, T_{21}<0, T_{22}>0, \\
& \tan \left(\theta_{2} / 2\right) / \tan \left(\theta_{1} / 2\right)=-T_{11} / T_{12}, \\
& \left\{\begin{array}{l}
-2\left\langle V_{1}, V_{2}\right\rangle \cos \frac{\theta_{1}}{2} \cos \frac{\theta_{2}}{2}+2\left\langle e_{1}, e_{2}\right\rangle \sin \frac{\theta_{1}}{2} \sin \frac{\theta_{2}}{2}>2, \\
-2\left\langle V_{1}^{\prime}, V_{2}^{\prime}\right\rangle \cos \frac{\theta_{1}}{2} \cos \frac{\theta_{2}}{2}+2\left\langle e_{1}^{\prime}, e_{2}^{\prime}\right\rangle \sin \frac{\theta_{1}}{2} \sin \frac{\theta_{2}}{2}>2 .
\end{array}\right.
\end{aligned}
$$

Proof. Consider a representation $\rho \in \mathcal{P}_{\alpha_{1}, \alpha_{2} ; \beta_{1}, \beta_{2}}$. Then the traces of $\rho \alpha_{i}$ are real and $\theta_{i} \in(0, \pi)$, so we have (3) and (4). Also, the traces of $\rho \beta_{i}$ are real, so from Theorem 3.1 we have

$$
\left\{\begin{array}{l}
\operatorname{tr}\left(T_{L_{1}}\left(\tau_{1}\right) T_{M}(d) T_{L_{1}}\left(\tau_{2}\right) T_{M}(d)\right) \in \mathbb{R} \\
\operatorname{tr}\left(T_{L_{1}}\left(\tau_{1}-l_{1}\right) T_{M}(d) T_{L_{1}}\left(\tau_{2}+l_{2}\right) T_{M}(d)\right) \in \mathbb{R}
\end{array}\right.
$$

Using Proposition 3.2 to compute the imaginary parts, the above conditions are equivalent to

$$
\left\{\begin{array}{l}
\cos \frac{\theta_{1}}{2} \sin \frac{\theta_{2}}{2}\left\langle V_{1}, e_{2}\right\rangle+\sin \frac{\theta_{1}}{2} \cos \frac{\theta_{2}}{2}\left\langle e_{1}, V_{2}\right\rangle=0 \\
\cos \frac{\theta_{1}}{2} \sin \frac{\theta_{2}}{2}\left\langle V_{1}^{\prime}, e_{2}^{\prime}\right\rangle+\sin \frac{\theta_{1}}{2} \cos \frac{\theta_{2}}{2}\left\langle e_{1}^{\prime}, V_{2}^{\prime}\right\rangle=0
\end{array}\right.
$$

Dividing by $\cos \left(\theta_{1} / 2\right) \sin \left(\theta_{2} / 2\right)$, we get that $\rho$ satisfies this system if and only if $\operatorname{det} T=0$ and

$$
\frac{\tan \left(\theta_{2} / 2\right)}{\tan \left(\theta_{1} / 2\right)}=K, \quad \text { where } \quad K=-\frac{\left\langle e_{1}, V_{2}\right\rangle}{\left\langle V_{1}, e_{2}\right\rangle} \quad \text { or } \quad-\frac{\left\langle e_{1}^{\prime}, V_{2}^{\prime}\right\rangle}{\left\langle V_{1}^{\prime}, e_{2}^{\prime}\right\rangle}
$$

(Notice that, by (9), we have $\left\langle V_{1}, e_{2}\right\rangle=0$ if and only if $\left\langle e_{1}, V_{2}\right\rangle=0$, and $\left\langle V_{1}^{\prime}, e_{2}^{\prime}\right\rangle=0$ if and only if $\left\langle e_{1}^{\prime}, V_{2}^{\prime}\right\rangle=0$. Moreover, if $\left\langle V_{1}, e_{2}\right\rangle=\left\langle e_{1}, V_{2}\right\rangle=0$, then by Lemma 4.8 $\left\langle V_{1}^{\prime}, e_{2}^{\prime}\right\rangle \neq 0$. So, $K$ is well defined.) Since $\theta_{1}, \theta_{2}>0$, then (10) implies that $T_{11}, T_{12}$ have opposite signs, and so do $T_{21}, T_{22}$.

Now we define the $\alpha$-lines described above. Denote by $\mathcal{R}_{0}$ the subset of $\mathcal{F}$ defined by the conditions that $\operatorname{det} T=0$, that $T_{11}, T_{12}$ have opposite signs (possibly vanishing), and that $T_{21}, T_{22}$ have opposite signs. For a point $p \in \mathcal{R}_{0}$, the $\alpha$-line based at $p$ is the curve $\sigma:[0, \pi] \rightarrow \mathbb{C}^{4}$ defined by $l_{i}(\sigma(t))=l_{i}(p)$ and $t_{i}(\sigma(t))=t_{i}(p)$ for $i=1,2 ; \theta_{1}(\sigma(t))=t$ and $\theta_{2}(\sigma(t))=2 \arctan (K \tan (t / 2))$. Thus, along an $\alpha$ line, the length of $\alpha_{i}$ and the real part of the twist $t_{\alpha_{i}}$ remain constant, and the angle ratio $\tan \theta_{1} / 2: \tan \theta_{2} / 2$ is fixed. Notice that on an $\alpha$-line, the traces of $\rho \beta_{i}$ are real and the angles $\theta_{1}$ and $\theta_{2}$ have the same sign (since the base point $p$ is in $\left.\mathcal{R}_{0}\right)$.

There is a unique $\alpha$-line, $\sigma$ through our point $\rho \in \mathcal{P}_{\alpha_{1}, \alpha_{2} ; \beta_{1}, \beta_{2}}$. Denote by $\rho_{0}$ its base point in $\mathcal{F}$, so that $\sigma(0)=\rho_{0}$ and $\sigma\left(t_{*}\right)=\rho$ for some $t_{*}>0$. We shall show that $\rho_{0} \in \mathcal{S}_{\alpha_{1}, \alpha_{2} ; \beta_{1}, \beta_{2}}$ and that $\sigma\left(\left[0, t_{*}\right]\right) \subset \mathcal{P}_{\alpha_{1}, \alpha_{2} ; \beta_{1}, \beta_{2}}$.

For all $t \in\left(0, t_{*}\right]$, the lengths $l_{\alpha_{i}}$ are fixed and the angles $\theta_{i}$ are in $(0, \pi)$. At $\rho=\sigma\left(t_{*}\right)$, we have $l_{\beta_{i}}>0$, and therefore $\left|\operatorname{tr} \rho \beta_{i}\right|>2$. By Proposition 5.11 (a) below, actually $\operatorname{tr} \rho \beta_{i}>2$, and by part (d) of that proposition, $\operatorname{tr} \rho \beta_{i}>2$ for all $t \in\left(0, t_{*}\right]$. Therefore, along $\left.\sigma\right|_{\left(0, t_{*}\right]}$, the lengths of $\beta_{1}, \beta_{2}$ are strictly positive. Hence all points on $\left.\sigma\right|_{\left(0, t_{*}\right]}$ satisfy the hypotheses of Proposition 5.9 below. Since at $\rho$ we know $\theta_{\beta_{i}} \neq 0, \pm \pi$, parts (c) and (d) of that proposition show that neither row of 
the matrix $T$ vanishes. Now this matrix is constant along $\sigma$. Thus, applying the reciprocal of parts (c), (d), we have that $\theta_{\beta_{i}} \neq 0, \pm \pi$ along $\left.\sigma\right|_{\left(0, t_{*}\right]}$.

In conclusion, along $\left.\sigma\right|_{\left(0, t_{*}\right]}$ we have $l_{\alpha_{i}} \neq 0, l_{\beta_{i}} \neq 0, \theta_{\alpha_{i}} \neq 0, \pm \pi$, and $\theta_{\beta_{i}} \neq$ $0, \pm \pi$. Since $\sigma\left(t_{*}\right)=\rho$ is in the pleating variety $\mathcal{P}_{\alpha_{1}, \alpha_{2} ; \beta_{1}, \beta_{2}}$ we can apply the local and the limit pleating theorems as in Section 5.1 to conclude that $\left.\sigma\right|_{\left(0, t_{*}\right]} \subset$ $\mathcal{P}_{\alpha_{1}, \alpha_{2} ; \beta_{1}, \beta_{2}}$. By Theorem 5.1, $\rho_{0}$ must be in the simplex of minima $\overline{\mathcal{S}}_{\alpha_{1}, \alpha_{2} ; \beta_{1}, \beta_{2}}$. By Theorem [4.9, the signs of $T$ are $\left({ }_{-}^{+}\right)$, and all the signs are strict by Proposition 5.9 So $\rho$ satisfies the conditions ([6) and (7). Finally, condition (8) follows since $\operatorname{tr} \rho \beta_{i}>$ 2 , as explained above.

Conversely, suppose $\rho \in \mathbb{C}^{4}$ satisfies (3)-(8). We want to prove that $\rho \in$ $\mathcal{P}_{\alpha_{1}, \alpha_{2} ; \beta_{1}, \beta_{2}}$. Consider the $\alpha$-line $\sigma$ through $\rho$. By (5) and (6) its base point $\sigma(0)=\rho_{0}$ is in the simplex of minima $\mathcal{S}_{\alpha_{1}, \alpha_{2} ; \beta_{1}, \beta_{2}}$. Suppose $\sigma\left(t_{*}\right)=\rho$. Along the path $\left.\sigma\right|_{\left(0, t_{*}\right]}$, the traces of $\rho \alpha_{i}, \rho \beta_{i}$ are real by conditions (3), (5) and (7); the angles $\theta_{1}, \theta_{2} \in(0, \pi)$, by (4); the length of $\beta_{i}$ is positive, because it is true for $\sigma\left(t_{*}\right)=\rho$, by (8), and we can apply Proposition 5.11 (d); and the angles $\theta_{\beta_{i}}$ are different from $0, \pm \pi$, because $l_{\beta_{i}} \neq 0$, and we can apply Proposition 5.9. It remains only to see that $\theta_{\beta_{1}}, \theta_{\beta_{2}}$ have the same sign. To do this we use Lemma 5.2 For $\epsilon$ sufficiently small, $\left.\sigma\right|_{[0, \epsilon)}$ is a path in $\mathcal{Q F}$, with $\sigma(0) \in \mathcal{F}$. We observe that the limit as $t \rightarrow 0$ of $\theta_{2} / \theta_{1}$ exists. Then, by Lemma [5.2, the limit of $\theta_{\beta_{2}} / \theta_{\beta_{1}}$ also exists and is equal to $b_{2} / b_{1}$, where $\left(b_{1}, b_{2}\right)$ is a left nullvector of $M\left(\mathcal{A}, \mathcal{B}, \rho_{0}\right)$. Since $\rho_{0} \in \mathcal{S}_{\alpha_{1}, \alpha_{2} ; \beta_{1}, \beta_{2}}$, this matrix has rank one and by Theorem 4.9 its left nullvectors have coordinates of the same sign. Thus $b_{1}, b_{2}$ have the same sign, and therefore $\theta_{\beta_{1}}, \theta_{\beta_{2}}$ have also the same sign at $\sigma(t)$, for $t$ sufficiently small. Applying Theorems 5.4, 5.5 and 5.6 we conclude that $\left.\sigma\right|_{\left(0, t_{*}\right]} \subset \mathcal{P}_{\alpha_{1}, \alpha_{2} ; \beta_{1}, \beta_{2}}$.

In the remainder of this subsection we state and prove Propositions 5.9 and 5.11 We begin with a lemma which will be used in Proposition 5.9. Recall from page 625 that for disjoint curves $\gamma, \gamma^{\prime}$ with real trace, we denote by $\mathrm{Pl}_{\gamma, \gamma^{\prime}}$ the pleated surface made from the Fenchel Nielsen construction by gluing the flat hyperbolic pants with boundary curves $\gamma, \gamma^{\prime}$. The bending angles of this surface are the angles between the two planes. Such a surface is a component of $\partial \mathcal{C}$ if and only if it is convex and embedded.

Lemma 5.8. Let $\rho \in \mathcal{R}(S)$ with $\operatorname{tr} \rho \alpha_{i}, \operatorname{tr} \rho \beta_{i} \in \mathbb{R}$ and with $\left|\operatorname{tr} \rho \alpha_{i}\right|,\left|\operatorname{tr} \rho \beta_{i}\right|>2$. Denote by $\theta_{\alpha_{1}}, \theta_{\alpha_{2}}$ the bending angles of the pleated surface $\mathrm{Pl}_{\alpha_{1}, \alpha_{2}}$ and by $\theta_{\beta_{1}}, \theta_{\beta_{2}}$ the bending angles of the pleated surface $\mathrm{Pl}_{\beta_{1}, \beta_{2}}$. Then, the pair $\left(\theta_{\alpha_{1}}, \theta_{\alpha_{2}}\right)$ is in the set $X=\{(0,0),(0, \pm \pi),( \pm \pi, 0),( \pm \pi, \pm \pi)\}$ if and only if $\left(\theta_{\beta_{1}}, \theta_{\beta_{2}}\right)$ is also in $X$.

Proof. Since $\left|\operatorname{tr} \rho \alpha_{i}\right|$ is strictly greater than $2,\left(\theta_{\alpha_{1}}, \theta_{\alpha_{2}}\right) \in X$ is equivalent to the pleated surface $\mathrm{Pl}_{\alpha_{1}, \alpha_{2}}$ being contained in a plane $P_{0}$. Then $\rho \beta_{i}$ leaves $P_{0}$ invariant and is purely hyperbolic, so its axis is contained in $P_{0}$. Moreover, all images of this axis under the group are contained in $P_{0}$. Therefore, $\mathrm{Pl}_{\beta_{1}, \beta_{2}}$ are also contained in $P_{0}$, and hence $\left(\theta_{\beta_{1}}, \theta_{\beta_{2}}\right) \in X$.

Proposition 5.9. Let $\rho \in \mathcal{R}(S)$ with $\operatorname{tr} \rho \alpha_{i}, \operatorname{tr} \rho \beta_{i} \in \mathbb{R},\left|\operatorname{tr} \rho \alpha_{i}\right|,\left|\operatorname{tr} \rho \beta_{i}\right|>2$, and $\left(\theta_{\alpha_{1}}, \theta_{\alpha_{2}}\right) \notin X$. Then:

(a) $\theta_{\alpha_{2}}=0$ or $\theta_{\alpha_{1}}= \pm \pi$ if and only if $T_{11}=T_{21}=0$.

(b) $\theta_{\alpha_{1}}=0$ or $\theta_{\alpha_{2}}= \pm \pi$ if and only if $T_{12}=T_{22}=0$.

(c) $\theta_{\beta_{2}}=0$ or $\theta_{\beta_{1}}= \pm \pi$ if and only if $T_{11}=T_{12}=0$.

(d) $\theta_{\beta_{1}}=0$ or $\theta_{\beta_{2}}= \pm \pi$ if and only if $T_{21}=T_{22}=0$. 


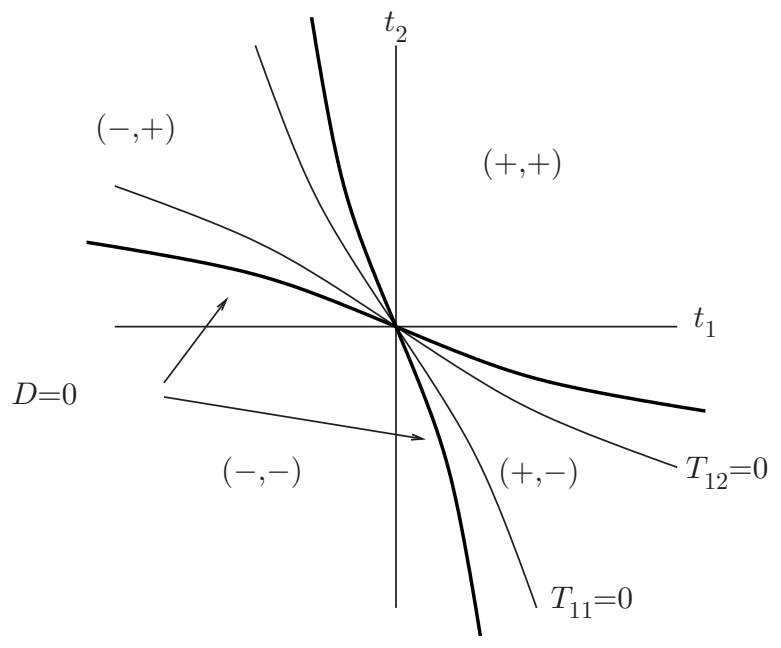

Figure 7 . The curve $D=0$ and the signs of $\left(T_{11}, T_{12}\right)$.

Proof. Since $\left|\operatorname{tr} \rho \alpha_{i}\right|>2$, we have $l_{\alpha_{i}}>0$, and we can write $\rho$ in terms of FenchelNielsen $\alpha$-coordinates. In these coordinates, the condition that the traces of $\rho \beta_{i}$ be real is the system (92). Suppose $\theta_{\alpha_{2}}=0$ or $\theta_{\alpha_{1}}= \pm \pi$. Then, (9) is equivalent to

$$
\sin \frac{\theta_{\alpha_{1}}}{2} \cos \frac{\theta_{\alpha_{2}}}{2} T_{11}=0 \text { and } \sin \frac{\theta_{\alpha_{1}}}{2} \cos \frac{\theta_{\alpha_{2}}}{2} T_{21}=0 .
$$

Since $\left(\theta_{\alpha_{1}}, \theta_{\alpha_{2}}\right) \notin X$, this is equivalent to $T_{11}=T_{21}=0$. For the converse, we observe that $T_{11}=T_{21}=0$ implies (see Lemma 4.8) that $T_{12}, T_{22} \neq 0$, so, from (9)) we must have $\theta_{\alpha_{1}}= \pm \pi$ or $\theta_{\alpha_{2}}=0$.

The other cases are similar; for (c), (d) we use Fenchel-Nielsen $\beta$-coordinates, and the system analogous to (9).

5.2.1. The $\alpha$-lines. In Proposition 5.11 we study the behaviour of the length of the curves $\beta_{1}, \beta_{2}$ along the $\alpha$-lines defined in the proof of Theorem 5.7. We begin with a lemma about signs, illustrated in Figure 7.

Lemma 5.10. Let $D=\left\langle e_{1}, e_{2}\right\rangle+1$. In the 2-plane in $\mathcal{F}$ defined by fixing concrete values for $l_{1}, l_{2}$, the curve $D=0$ does not intersect the regions $\mathcal{T}_{1}=\left\{T_{11}>0, T_{12}<\right.$ $0\}$ and $\mathcal{T}_{2}=\left\{T_{11}<0, T_{12}>0\right\}$. Moreover, $D$ is positive in both regions.

Proof. The 2-plane in the statement is coordinatized by the twist parameters $\left(t_{1}, t_{2}\right)$. From the formulae in Remark 3 of Section 3.1, we easily see that the graph $T_{11}=0$ intersects the $t_{i}$-axes at $(0,0)$ and that its tangent at this point has slope $-\cosh d$. Similarly, $T_{12}=0$ intersects the $t_{i}$-axes at $(0,0)$ and its tangent at this point has slope $-1 / \cosh d$. Also, the point $(0,0)$ is the only intersection point of these two curves. Thus it is easy to identify the regions $\mathcal{T}_{1}, \mathcal{T}_{2}$; see Figure 7 .

On the other hand, the curve $D=0$ intersects the axes only at $(0,0)$, and we have

$$
\frac{\partial D}{\partial t_{1}}=\frac{1}{2}\left\langle V_{1}, e_{2}\right\rangle, \quad \frac{\partial D}{\partial t_{2}}=\frac{1}{2}\left\langle e_{1}, V_{2}\right\rangle
$$

Both partial derivatives are zero if and only if $t_{1}=t_{2}=0$. Therefore, $(0,0)$ is the only singular point of $D$. 
By geometric arguments we can also see that $D=0$ intersects each of the curves $T_{11}=0$ and $T_{12}=0$ only in the point $(0,0)$ : with the notation of the Main Figure of Section 3.1, if $\left\langle e_{1}, V_{2}\right\rangle=0$ then $V_{2}$ is in the line $H_{1}$. Since $V_{2}$ is also in $H_{2}$, $H_{1} \cap H_{2} \neq \emptyset$. Now, $\left\langle e_{1}, e_{2}\right\rangle=-1$ implies that $H_{1}$ and $H_{2}$ coincide (the halfspaces $H_{1}^{-}, H_{2}^{-}$are opposite). This only happens when $t_{1}=t_{2}=0$.

Next, we study the sign of $T_{11}, T_{12}$ along the curve $D$. Referring again to the Main Figure, note that for each fixed $t_{1}$, there are at most two values of $t_{2}$ so that $\left(t_{1}, t_{2}\right) \in\{D=0\}$; see Figure 8. If $t_{1}$ is positive and large enough so that the line $H_{1}$ does not intersect $L_{2}$, then the value of $t_{2}$ is unique; see Figure $8(\mathrm{a})$. This value of $t_{2}$ is negative and at $\left(t_{1}, t_{2}\right)$ we have $T_{11}, T_{12}>0$. If $t_{1}>0$ but $H_{1}$ does intersect $L_{2}$, then there exist exactly two values of $t_{2}$ so that $\left(t_{1}, t_{2}\right) \in\{D=0\}$, both negative, one greater than $-t_{1}$ and the other less; see Figures $8(\mathrm{~b}), 8(\mathrm{c})$. For the first one, we have $T_{11}, T_{12}<0$ and for the second we have $T_{11}, T_{12}>0$. Similarly, we study the situation for negative values of $t_{1}$. This information allows one to verify the configuration of regions shown in Figure 7.

To study the sign of $D$ in the regions $\mathcal{T}_{1}, \mathcal{T}_{2}$, we just pick a point in each of these regions and compute the sign. For instance, if $t_{1}>0$ and $t_{2}=-t_{1}$, then $\left(t_{1}, t_{2}\right) \in \mathcal{T}_{1}$, and

$$
\left\langle e_{1}, e_{2}\right\rangle+1=\left(\cosh ^{2}\left(t_{1} / 2\right)-1\right)(\cosh d-1),
$$

which is positive. Thus $D$ is positive in $\mathcal{T}_{1}$ and, similarly, in $\mathcal{T}_{2}$.

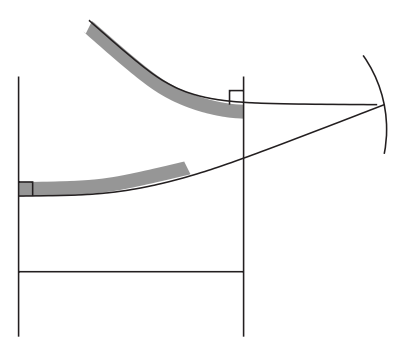

(a) $t_{1} \gg 0, T_{11}, T_{22}>0$

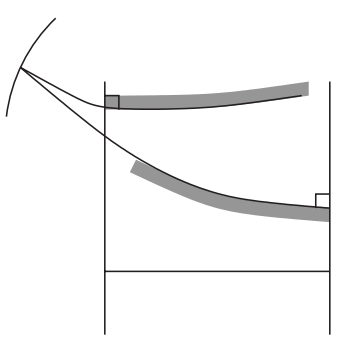

(b) $t_{1}>0, T_{11}, T_{12}<0$

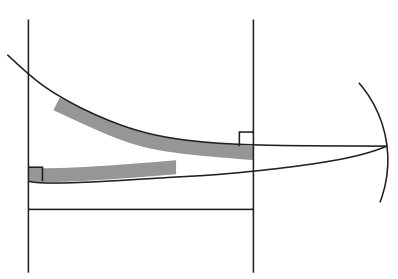

(c) $t_{1}>0, T_{11}, T_{12}>0$

Figure 8.

Now we can prove the proposition we need.

Proposition 5.11. Let $p \in \mathcal{R}_{0}$ and consider the $\alpha$-line $\sigma$ based on $p$. Consider along $\sigma$ the functions

$$
\begin{aligned}
& f_{1}=\operatorname{tr} \rho \beta_{1}=-2\left\langle V_{1}, V_{2}\right\rangle \cos \frac{\theta_{1}}{2} \cos \frac{\theta_{2}}{2}+2\left\langle e_{1}, e_{2}\right\rangle \sin \frac{\theta_{1}}{2} \sin \frac{\theta_{2}}{2} \\
& f_{2}=\operatorname{tr} \rho \beta_{2}=-2\left\langle V_{1}^{\prime}, V_{2}^{\prime}\right\rangle \cos \frac{\theta_{1}}{2} \cos \frac{\theta_{2}}{2}+2\left\langle e_{1}^{\prime}, e_{2}^{\prime}\right\rangle \sin \frac{\theta_{1}}{2} \sin \frac{\theta_{2}}{2} .
\end{aligned}
$$

Then for each $i=1,2$ :

(a) $f_{i}\left(\theta_{1}\right) \geq-2$ for all $\theta_{1} \in[0, \pi]$.

(b) If $\left\langle e_{1}, e_{2}\right\rangle \geq 1$, then $f_{1}\left(\theta_{1}\right) \geq 2$ for all $\theta_{1}$; likewise if $\left\langle e_{1}^{\prime}, e_{2}^{\prime}\right\rangle \geq 1$, then $f_{2}\left(\theta_{1}\right) \geq 2$.

(c) $f_{i}$ is decreasing near 0 and has at most one critical point (which will be a minimum) in $(0, \pi)$. 
(d) If for some $t_{*}$ we have $f_{i}\left(t_{*}\right)>2$, then $f_{i}(t)>2$ for all $t<t_{*}$. If there exists $t_{*}$ so that $f_{i}\left(t_{*}\right)=2$, then $f_{i}$ is decreasing in the interval $\left[0, t_{*}\right]$.

Proof. We prove this result for $f_{1}$; the proof for $f_{2}$ is analogous.

(a) Since $p \in \mathcal{R}_{0}$, by Lemma 5.10 we have $\left\langle e_{1}, e_{2}\right\rangle \geq-1$. Then the assertion is an immediate consequence (remember that $\left\langle V_{1}, V_{2}\right\rangle=-\cosh \mathrm{d}\left(V_{1}, V_{2}\right)<0$ ).

(b) Since we already know that $\operatorname{tr} \rho \beta_{1}$ is real, we just need to show that $p \circ \rho \beta_{1}$ is not an elliptic isometry, for any value of $\theta_{1}$. For this, it is enough to decompose $p \circ \rho \beta_{1}$ as a product of two involutions around disjoint axes. We use a geometrical argument similar to that in Remark 2 in Section 3.1, but first, we need to elaborate the picture to take account of the fact that the twist parameters $\tau_{1}=t_{1}+i \theta_{1}, \tau_{2}=$ $t_{2}+i \theta_{2}$ are now complex. We regard the Main Figure in Section 3.1 as a plane $H$ contained in $\mathbb{H}^{3}$. Let $P_{1}, P_{2}$ be the orthogonal lines to $H$ through the points $O_{1}, O_{2}$, oriented so that $P_{i}, M, L_{i}$ give the orientation of $\mathbb{H}^{3}$. Let $W_{1}\left(\tau_{1}\right)$ be the line so that the complex distance $\mathbf{d}_{L_{1}}\left(P_{1}, W_{1}\left(\tau_{1}\right)\right)$ is $\tau_{1} / 2$, and $W_{2}\left(\tau_{2}\right)$ the line so that $\mathbf{d}_{L_{2}}\left(P_{2}, W_{2}\left(\tau_{2}\right)\right)$ is $-\tau_{2} / 2$. Then, by decomposing isometries of $\mathbb{H}^{3}$ as products of involutions around axes, we find the isometry

$$
p \circ \rho\left(\beta_{1}\right)=\bar{T}_{L_{1}}\left(\tau_{1}\right) \bar{T}_{M}(d) \bar{T}_{L_{1}}\left(\tau_{2}\right) \bar{T}_{M}(d)=I_{W_{1}\left(\tau_{1}\right)} I_{W_{2}\left(\tau_{2}\right)},
$$

where now $I_{N}$ means involution around the hyperbolic line $N$.

Now consider the plane $\Pi_{i}$ orthogonal to $L_{i}$ at $V_{i}$ (so, containing the line $H_{i}$ ), for $i=1,2$. Then $\Pi_{1} \cap \Pi_{2}=\emptyset$ if and only if $H_{1} \cap H_{2}=\emptyset$. If $\left\langle e_{1}, e_{2}\right\rangle>1$, then $H_{1}$, $H_{2}$ do not intersect, and therefore neither do $\Pi_{1}, \Pi_{2}$. Now, the line $W_{1}\left(\tau_{1}\right)$ defined above is contained in the plane $\Pi_{1}$, and $W_{2}\left(\tau_{2}\right)$ is contained in $\Pi_{2}$, and therefore the two lines are disjoint. Hence $p \circ \rho\left(\beta_{1}\right)$ is purely hyperbolic and $\left|\operatorname{tr} \rho\left(\beta_{1}\right)\right|>2$. (If $\left\langle e_{1}, e_{2}\right\rangle=1$, it might be parabolic for some value of $\theta_{1}$.) Since $f_{1}=\operatorname{tr} \rho \beta_{1}$ is continuous and $f_{1}(0)$ is positive, it follows that $f_{1}\left(\theta_{1}\right) \geq 2$ for all $\theta_{1}$.

(c) As in the proof of Theorem 5.7 let $K=-\left\langle e_{1}, V_{2}\right\rangle /\left\langle V_{1}, e_{2}\right\rangle$, and set also $A=$ $-\left\langle V_{1}, V_{2}\right\rangle$ and $B=\left\langle e_{1}, e_{2}\right\rangle$. We regard $f_{1}$ as a function on $\theta_{1} \in[0, \pi]$, and compute its derivative. We have that $f_{1}^{\prime}(0)=0, f_{1}^{\prime}(\pi)=0$. Since $\tan \left(\theta_{2} / 2\right)=K \tan \left(\theta_{1} / 2\right)$, the derivative $\theta_{2}^{\prime}\left(\theta_{1}\right)$ is

$$
\theta_{2}^{\prime}=K\left(1+\tan ^{2}\left(\theta_{1} / 2\right)\right)\left(1+K^{2} \tan ^{2}\left(\theta_{1} / 2\right)\right)^{-1} .
$$

Then, for $\theta_{1} \neq 0, \pi$, we have

$$
f_{1}^{\prime}\left(\theta_{1}\right)\left(\cos \left(\theta_{1} / 2\right)\right)^{-2}=\tan \left(\theta_{1} / 2\right)\left(-A-A K \theta_{2}^{\prime}+B K+B \theta_{2}^{\prime}\right) .
$$

If $\theta_{1} \neq 0, \pi$, then $f_{1}^{\prime}=0$ if and only if $-A-A K \theta_{2}^{\prime}+B K+B \theta_{2}^{\prime}=0$, and this is equivalent to

$$
\tan ^{2}\left(\theta_{1} / 2\right)=\left(A-2 B K+A K^{2}\right)\left(K\left(B-2 A K+B K^{2}\right)\right)^{-1}
$$

which has at most one solution for $\theta_{1} \in(0, \pi)$.

Next we study the sign of $f_{1}^{\prime}\left(\theta_{1}\right)$ for $\theta_{1}$ near 0 . From (12), for $\theta_{1}$ positive and near 0 , the sign of $f_{1}^{\prime}$ is the same as the sign of $g\left(\theta_{1}\right)=-A-A K \theta_{2}^{\prime}+B K+B \theta_{2}^{\prime}$. Now, $\theta_{2}^{\prime}(0)=K$, and therefore,

$$
g(0) /(2 K)=-A\left(1+K^{2}\right)(2 K)^{-1}+B<-A+B=\left\langle V_{1}, V_{2}\right\rangle+\left\langle e_{1}, e_{2}\right\rangle,
$$

where the inequality holds because $A$ is positive and $\left(1+K^{2}\right) / 2 K>1$. Now $\left\langle V_{1}, V_{2}\right\rangle+\left\langle e_{1}, e_{2}\right\rangle$ is negative, as explained in the proof of Proposition 4.12. Hence, $g\left(\theta_{1}\right)$ and $f_{1}^{\prime}\left(\theta_{1}\right)$ are negative, for $\theta_{1}$ near 0 . 
The final result $(\mathrm{d})$ now follows easily. If $\left\langle e_{1}, e_{2}\right\rangle \leq 0$, then $f_{1}$ is decreasing, and the result is clear; if $0 \leq\left\langle e_{1}, e_{2}\right\rangle<1$, then $f_{1}(\pi)<2$, and the result follows from (c); otherwise, apply (b).

Non-singularity. We end this subsection by proving that the lengths of the bending lines are local coordinates in a neighbourhood of $\mathcal{P}_{\alpha_{1}, \alpha_{2} ; \beta_{1}, \beta_{2}}$. First we examine the situation in $\mathcal{F}$.

Proposition 5.12. Let $p_{0} \in \mathcal{S}_{\alpha_{1}, \alpha_{2} ; \beta_{1}, \beta_{2}}$, with $l_{\alpha_{i}}\left(p_{0}\right)=c_{i}, i=1,2$, and let $W \subset \mathcal{F}$ be the subset defined by $l_{\alpha_{i}}(p)=c_{i}, i=1,2$. Then $W \cap \mathcal{S}_{\alpha_{1}, \alpha_{2} ; \beta_{1}, \beta_{2}}$ is locally homeomorphic to $\mathbb{R}$.

Proof. Let $\mathcal{Q}_{\mathcal{A}}, \mathcal{Q}_{\mathcal{B}} \subset \mathcal{M L}$ denote respectively those laminations $\mu=a_{1} \alpha_{1}+a_{2} \alpha_{2}$ and $\nu=b_{1} \beta_{1}+b_{2} \beta_{2}$ for which $a_{1}+a_{2}=b_{1}+b_{2}=1$ and all coefficients are positive. Denote by $\left[\mathcal{Q}_{\mathcal{B}}\right]$ the projection of $\mathcal{Q}_{\mathcal{B}}$ on P $\mathcal{M L}$. By Theorem 2.1 in [15], if $j: \mathrm{P} \mathcal{M L} \rightarrow \mathcal{M L}$ is any fixed section, then for each $p \in \mathcal{F}$ and $\mu \in \mathcal{Q}_{\mathcal{A}}$ there exist unique $[\xi] \in \mathrm{P} \mathcal{M L}$ and $k \in(0,1)$ such that $(1-k) l_{\mu}+k l_{j([\xi])}$ takes its minimum at $p$. By Theorem 7.7 of [20], the resulting map $\Psi: W \times \mathcal{Q}_{\mathcal{A}} \rightarrow \mathrm{P} \mathcal{M} \mathcal{L}$, defined by $\Psi(p, \mu)=[\xi]$, is injective, and hence, since both domain and range have the same real dimension 3 , open. Since $p_{0} \in \mathcal{S}_{\alpha_{1}, \alpha_{2} ; \beta_{1}, \beta_{2}}$, then certainly [ $\mathcal{Q}_{\mathcal{B}}$ ] intersects the image of $\Psi$. Thus, since $\left[\mathcal{Q}_{\mathcal{B}}\right]$ is homeomorphic to an open interval, so locally is $\Psi^{-1}\left(\left[\mathcal{Q}_{\mathcal{B}}\right]\right) \subset W \times \mathcal{Q}_{\mathcal{A}}$. From the definitions, if $p \in W$ and $\mu \in \mathcal{Q}_{\mathcal{A}}$, then $\Psi(p, \mu) \in\left[\mathcal{Q}_{\mathcal{B}}\right]$ implies $p \in \mathcal{S}_{\alpha_{1}, \alpha_{2} ; \beta_{1}, \beta_{2}}$. Thus $\Psi^{-1}\left(\left[\mathcal{Q}_{\mathcal{B}}\right]\right) \subset\left(W \cap \mathcal{S}_{\alpha_{1}, \alpha_{2} ; \beta_{1}, \beta_{2}}\right) \times \mathcal{Q}_{\mathcal{A}}$.

We now claim that the projection $\Psi^{-1}\left(\left[\mathcal{Q}_{\mathcal{B}}\right]\right) \rightarrow W \cap \mathcal{S}_{\alpha_{1}, \alpha_{2} ; \beta_{1}, \beta_{2}}$ is injective. This is because if $(p, \mu),\left(p, \mu^{\prime}\right) \in \Psi^{-1}\left(\left[\mathcal{Q}_{\mathcal{B}}\right]\right)$, then $p$ lies on two lines of minima $\mathcal{L}_{\mu, \nu}$ and $\mathcal{L}_{\mu^{\prime}, \nu^{\prime}}$ with $\nu, \nu^{\prime} \in \mathcal{Q}_{\mathcal{B}}$. However, by Proposition 4.6 (a) and Theorem 4.9 the map $\Phi: \mathcal{Q}_{\mathcal{A}} \times \mathcal{Q}_{\mathcal{B}} \times(0,1) \rightarrow \mathcal{S}_{\alpha_{1}, \alpha_{2} ; \beta_{1}, \beta_{2}}$ which sends $(\mu, \nu, k)$ to the minimum of $(1-k) l_{\mu}+k l_{\nu}$ is injective, so $p$ lies on exactly one line of minima $\mathcal{L}_{\mu, \nu}$. Since the projection is also open, we deduce that $W \cap \mathcal{S}_{\alpha_{1}, \alpha_{2} ; \beta_{1}, \beta_{2}}$ is locally homeomorphic to $\mathbb{R}$, as claimed.

Theorem 5.13. The length functions $\left(\lambda_{\alpha_{1}}(q), \lambda_{\alpha_{2}}(q), \lambda_{\beta_{1}}(q), \lambda_{\beta_{2}}(q)\right)$ are local holomorphic coordinates for $\mathcal{Q} \mathcal{F}$ in a neighbourhood of $\mathcal{P}_{\alpha_{1}, \alpha_{2} ; \beta_{1}, \beta_{2}}$.

Proof. We have to prove that the map

$$
q \mapsto\left(\lambda_{\alpha_{1}}(q), \lambda_{\alpha_{2}}(q), \lambda_{\beta_{1}}(q), \lambda_{\beta_{2}}(q)\right)
$$

is invertible in a neighbourhood of each point in $\mathcal{P}_{\alpha_{1}, \alpha_{2} ; \beta_{1}, \beta_{2}}$.

Let $q_{0} \in \mathcal{P}_{\alpha_{1}, \alpha_{2} ; \beta_{1}, \beta_{2}}$ and let $\lambda_{\alpha_{i}}\left(q_{0}\right)=c_{i}, i=1,2$. Let $V$ be the submanifold of $\mathcal{Q F}$ defined by $\lambda_{\alpha_{i}}(q)=c_{i}, i=1,2$ (so that $V$ is locally $\mathbb{C}^{2}$ with coordinates $\left.\left(\tau_{\alpha_{1}}, \tau_{\alpha_{2}}\right)\right)$. Then, working with Fenchel-Nielsen $\alpha$-coordinates, it will be sufficient to prove that the map on $V$ defined by $\left(\tau_{\alpha_{1}}, \tau_{\alpha_{2}}\right) \mapsto\left(\lambda_{\beta_{1}}, \lambda_{\beta_{2}}\right)$ is non-singular (has non-vanishing Jacobian) at $q_{0}$. First, notice that each of the four derivatives $\frac{\partial \lambda_{\beta_{i}}}{\partial \tau_{\alpha_{j}}}$ is non-zero, because by the generalization of Kerckhoff's derivative formula to $\mathcal{Q} \mathcal{F}$, each consists of a single term $\cosh D_{i, j}\left(q_{0}\right)$, where $D_{i, j}$ is the complex distance between the axes of $\alpha_{i}$ and $\beta_{j}$. Since $q_{0} \notin \mathcal{F}$, the real part of this distance is certainly non-zero, and so $\cosh D_{i, j}\left(q_{0}\right) \neq 0$. Thus, in particular, the map $\left(\tau_{\alpha_{1}}, \tau_{\alpha_{2}}\right) \mapsto\left(\lambda_{\beta_{1}}, \tau_{\alpha_{2}}\right)$ is non-singular at $q_{0}$, and so it remains to prove that the map $F:\left(\lambda_{\beta_{1}}, \tau_{\alpha_{2}}\right) \mapsto\left(\lambda_{\beta_{1}}, \lambda_{\beta_{2}}\right)$ is non-singular at $q_{0}$. In other words, regarding $\lambda_{\beta_{2}}$ as a function of $\lambda_{\beta_{1}}$ and $\tau_{\alpha_{2}}$, we have to show that $\partial \lambda_{\beta_{2}} / \partial \tau_{\alpha_{2}} \neq 0$. To abbreviate notation, write $z=\lambda_{\beta_{1}}-\lambda_{\beta_{1}}\left(q_{0}\right), w=\tau_{\alpha_{2}}-\tau_{\alpha_{2}}\left(q_{0}\right)$, and view these as local 
coordinates for $V$ near $q_{0}$. Using these coordinates, write $f=\lambda_{\beta_{2}}-\lambda_{\beta_{2}}\left(q_{0}\right)$. Thus we have $f(0,0)=0$, and we have to prove that the map $F:(z, w) \mapsto(z, f(z, w))$ is non-singular at $(0,0)$.

Let $\mathcal{P}=\mathcal{P}_{\alpha_{1}, \alpha_{2} ; \beta_{1}, \beta_{2}} \cap V$. We first claim that $\mathcal{P}$ is locally homeomorphic to $\mathbb{R}^{2}$. In fact, by Proposition $5.12 W \cap \mathcal{S}_{\alpha_{1}, \alpha_{2} ; \beta_{1}, \beta_{2}}$ is locally homeomorphic to $\mathbb{R}$, where obviously $W=V \cap \mathcal{F}$. The claim then follows easily from Theorem 5.7 which, as pointed out, shows that the pleating variety is just the product of $\mathcal{S}_{\alpha_{1}, \alpha_{2} ; \beta_{1}, \beta_{2}}$ with an open interval, with the fibres over the points of $\mathcal{S}_{\alpha_{1}, \alpha_{2} ; \beta_{1}, \beta_{2}}$ being the $\alpha$-lines.

Since $\mathcal{P}$ is foliated by $\alpha$-lines, it is locally parameterised by $\left(\zeta, \theta_{\alpha_{1}}\right)$, with $\zeta$ the parameter of $V \cap \mathcal{F}$ and $\theta_{\alpha_{1}}$ the imaginary part of $\tau_{\alpha_{1}}$, which parameterises the $\alpha$-lines. Let $\sigma$ be any $\alpha$-line. We claim that at most one of the real functions $f_{i}=\operatorname{tr} \rho \beta_{i}(i=1,2)$ has a critical point on $\sigma$. In fact, by Theorem 5.7, following $\sigma$ from its initial point in $\mathcal{F}$, we remain in $\mathcal{P}$ until the first point at which one of the $f_{i}$, say $f_{1}$, reaches the value 2 . Proposition 5.11 (d) then asserts that $f_{1}$ has no critical point on $\sigma$. In particular, the function $z=\lambda_{\beta_{1}}-\lambda_{\beta_{1}}\left(q_{0}\right)$, restricted to $\mathcal{P}$, has no critical point on the $\alpha$-line $\sigma$ containing $q_{0}$; then it follows from the implicit function theorem on $\mathcal{P}$ that, in a neighbourhood of $q_{0}$, the locus $\{z=0\} \cap \mathcal{P}$ is the graph of some function $\zeta=h\left(\theta_{\alpha_{1}}\right)$.

We first rule out the possibility that $f(z, w)$ vanishes identically on $z=0$. Notice that by the local pleating theorem 5.5] there is a neighbourhood $U$ of $q_{0}$ in $V$ such that $F^{-1}(\mathbb{R} \times \mathbb{R}) \cap U=\mathcal{P} \cap U$, and hence, from what we have already proved about $\mathcal{P}$, it follows that $F^{-1}(\mathbb{R} \times \mathbb{R}) \cap U$ is locally homeomorphic to $\mathbb{R}^{2}$. On the other hand, if $f(z, w)$ vanishes identically on $z=0$, then $F^{-1}(\mathbb{R} \times \mathbb{R}) \cap U$ contains (a) a neighbourhood $U_{0}=\{(0, w):|w|<\epsilon\}$ of $(0,0)$ in $\{0\} \times \mathbb{C}$ and (b) a neighbourhood of $q_{0}$ on the $\alpha$-line through $q_{0}$ which intersects $U_{0}$ in at most one point (since $z$ is non-constant on $\alpha$-lines $)$. Thus in this case, $F^{-1}(\mathbb{R} \times \mathbb{R}) \cap U$ is not locally $\mathbb{R}^{2}$.

Thus we may assume that $f(z, w)$ does not vanish identically on $z=0$. By the Weierstrass preparation theorem we can write

$$
f(z, w)=\left(w^{m}+a_{1}(z) w^{m-1}+\ldots+a_{m}(z)\right) g(z, w)
$$

for some $m \in \mathbb{N}$, where $a_{i}(0)=0$ for all $i$ and $g(0,0) \neq 0$. Then $f(0, w)=$ $w^{m} g(0, w)$. It follows that $F^{-1}(0, \mathbb{R})$ consists of $m$ branches; more precisely, there are $m$ maps $(-\epsilon, \epsilon) \rightarrow F^{-1}(0, \mathbb{R}) \subset(\{z=0\} \cap \mathcal{P})$ whose images are disjoint except at 0 . By the above description of $\{z=0\} \cap \mathcal{P}$, this is clearly impossible unless $m=1$. Thus $\frac{\partial f}{\partial w}(0,0) \neq 0$ and $F$ is non-singular at $(0,0)$, as required.

5.3. Subpleating varieties of $\mathcal{P}_{\alpha_{1}, \alpha_{2} ; \beta_{1}, \beta_{2}}$. We now study the pleating varieties on the boundary of $\mathcal{P}_{\alpha_{1}, \alpha_{2} ; \beta_{1}, \beta_{2}}$ and also the special pleating plane $\mathcal{P}_{\mu, \nu}$ defined by fixing the measured laminations $\mu=\alpha_{1}+\alpha_{2}$ and $\nu=\beta_{1}+\beta_{2}$ as the bending lines, which is clearly contained in the interior of $\mathcal{P}_{\alpha_{1}, \alpha_{2} ; \beta_{1}, \beta_{2}}$.

Proposition 5.14. The boundary of $\mathcal{P}_{\alpha_{1}, \alpha_{2} ; \beta_{1}, \beta_{2}}$ in $\mathcal{Q} \mathcal{F}-\mathcal{F}$ consists of the pleating varieties $\mathcal{P}_{\alpha_{1} ; \beta_{1}, \beta_{2}}, \mathcal{P}_{\alpha_{2} ; \beta_{1}, \beta_{2}}, \mathcal{P}_{\alpha_{1}, \alpha_{2} ; \beta_{1}}$ and $\mathcal{P}_{\alpha_{1}, \alpha_{2} ; \beta_{2}}$. These pleating varieties are the set of representations $\rho$ which in terms of the $\alpha$-coordinates $\left(l_{1}+i \varphi_{1}, l_{2}+i \varphi_{2}\right.$, $\left.t_{1}+i \theta_{1}, t_{2}+i \theta_{2}\right)$ are described by:

(a) $\mathcal{P}_{\alpha_{1} ; \beta_{1}, \beta_{2}}$ is the set of points satisfying (两), $0<\theta_{1}<\pi, \theta_{2}=0, T_{11}=T_{21}=0$, and $\cos \left(\theta_{1} / 2\right)>\max \left\{-1 /\left\langle V_{1}, V_{2}\right\rangle,-1 /\left\langle V_{1}^{\prime}, V_{2}^{\prime}\right\rangle\right\}$.

(b) $\mathcal{P}_{\alpha_{2} ; \beta_{1}, \beta_{2}}$ is the set of points satisfying (3), $\theta_{1}=0,0<\theta_{2}<\pi, T_{12}=T_{22}=0$, and $\cos \left(\theta_{2} / 2\right)>\max \left\{-1 /\left\langle V_{1}, V_{2}\right\rangle,-1 /\left\langle V_{1}^{\prime}, V_{2}^{\prime}\right\rangle\right\}$. 
(c) $\mathcal{P}_{\alpha_{1}, \alpha_{2} ; \beta_{1}}$ is the set of points satisfying (3), (4), $T_{11}=T_{12}=0$,

$$
\frac{\tan \frac{\theta_{2}}{2}}{\tan \frac{\theta_{1}}{2}}=-\frac{T_{21}}{T_{22}}
$$

and

$$
2 \cosh d \cos \frac{\theta_{1}}{2} \cos \frac{\theta_{2}}{2}-2 \sin \frac{\theta_{1}}{2} \sin \frac{\theta_{2}}{2}>2
$$

(d) $\mathcal{P}_{\alpha_{1}, \alpha_{2} ; \beta_{2}}$ is the set of points satisfying (3), (4),$T_{21}=T_{22}=0$,

$$
\frac{\tan \frac{\theta_{2}}{2}}{\tan \frac{\theta_{1}}{2}}=-\frac{T_{11}}{T_{12}}
$$

and

$$
2 \cosh d \cos \frac{\theta_{1}}{2} \cos \frac{\theta_{2}}{2}-2 \sin \frac{\theta_{1}}{2} \sin \frac{\theta_{2}}{2}>2 .
$$

Proof. The boundary of $\mathcal{P}_{\alpha_{1}, \alpha_{2} ; \beta_{1}, \beta_{2}}$ in $\mathcal{Q F}-\mathcal{F}$ is the subset described, because if $\rho$ is in this boundary, then the lengths of $\rho \alpha_{i}$ and $\rho \beta_{i}$ are positive, and in this situation, the bending angles $\theta_{\alpha_{i}}$ and $\theta_{\beta_{i}}$ cannot be equal to $\pm \pi$. Therefore, the only possibility is that one of the bending angles is equal to zero. This gives the four subsets of the statement.

The converse parts of (a)-(d) are analogous to that of Theorem 5.7, using Proposition 5.9 to translate the equations of the form $T_{i j}=T_{h k}=0$ in terms of some bending angle being zero. In the case (c), we have $T_{11}=T_{12}=0$, which is equivalent to $t_{1}=t_{2}=0$. In this case, we have $-\left\langle V_{1}, V_{2}\right\rangle=\cosh d,\left\langle e_{1}, e_{2}\right\rangle=-1$, while $-\left\langle V_{1}^{\prime}, V_{2}^{\prime}\right\rangle>\cosh d$ and $\left\langle e_{1}^{\prime}, e_{2}^{\prime}\right\rangle=1$. Then, from the formulae for $\operatorname{tr} \rho \beta_{1}, \operatorname{tr} \rho \beta_{2}$, we see that $l_{\beta_{1}}$ is always smaller than $l_{\beta_{2}}$ along the $\alpha$-lines. Therefore the condition analogous to (8) is just one inequality, and similarly for the case (d).

We now compute the pleating plane $\mathcal{P}_{\mu, \nu}$ defined by fixing the measured laminations $\mu=\alpha_{1}+\alpha_{2}$ and $\nu=\beta_{1}+\beta_{2}$ as the bending lines. This is the subset of the pleating variety $\mathcal{P}_{\alpha_{1}, \alpha_{2} ; \beta_{1}, \beta_{2}}$ given by the extra conditions $\theta_{1}=\theta_{2}$ and $\theta_{\beta_{1}}=\theta_{\beta_{2}}$.

Proposition 5.15. The pleating plane $\mathcal{P}_{\mu, \nu}$ is the subset of points $\left(l_{1}+i \varphi_{1}, l_{2}+\right.$ $\left.\varphi_{2}, t_{1}+i \theta_{1}, t_{2}+i \theta_{2}\right) \in \mathbb{C}^{4}$ so that $\varphi_{1}=\varphi_{2}=0, l_{1}=l_{2}, t_{1}=-t_{2}=l_{1} / 2, \theta_{1}=\theta_{2}$ and

$$
0<\theta_{1}<\pi, \quad \cos ^{2}\left(\theta_{1} / 2\right)>\left(1-\left\langle e_{1}, e_{2}\right\rangle\right)\left(-\left\langle V_{1}, V_{2}\right\rangle-\left\langle e_{1}, e_{2}\right\rangle\right)^{-1} .
$$

Proof. If $\rho \in \mathcal{P}_{\mu, \nu} \subset \mathcal{P}_{\alpha_{1}, \alpha_{2} ; \beta_{1}, \beta_{2}}$, then, by Theorem [5.7, $\rho$ satisfies the system (9). Since $\theta_{1}=\theta_{2}$, and $\theta_{1} \neq 0, \pm \pi$, we must have

$$
\left\langle V_{1}, e_{2}\right\rangle+\left\langle e_{1}, V_{2}\right\rangle=0 \text { and }\left\langle V_{1}^{\prime}, e_{2}^{\prime}\right\rangle+\left\langle e_{1}^{\prime}, V_{2}^{\prime}\right\rangle=0 .
$$

As we have seen in the proof of Proposition 4.12, this is equivalent to $t_{1}=-t_{2}$, and $l_{1}=l_{2}$.

By the symmetry of the curves $\left\{\alpha_{1}, \alpha_{2}\right\},\left\{\beta_{1}, \beta_{2}\right\}$, the condition $\theta_{\beta_{1}}=\theta_{\beta_{2}}$ will imply $l_{\beta_{1}}=l_{\beta_{2}}$, or, equivalently, $\operatorname{tr} \rho \beta_{1}=\operatorname{tr} \rho \beta_{2}$ (these traces are already real). Computing these traces by Proposition [3.2, this condition is equivalent to

$$
\left(-\left\langle V_{1}, V_{2}\right\rangle+\left\langle V_{1}^{\prime}, V_{2}^{\prime}\right\rangle\right) \cos ^{2}\left(\theta_{1} / 2\right)+\left(\left\langle e_{1}, e_{2}\right\rangle-\left\langle e_{1}^{\prime}, e_{2}^{\prime}\right\rangle\right) \sin ^{2}\left(\theta_{1} / 2\right)=0 .
$$

Using that $t_{1}=-t_{2}, l_{1}=l_{2}$, we see that the functions $-\left\langle V_{1}, V_{2}\right\rangle+\left\langle V_{1}^{\prime}, V_{2}^{\prime}\right\rangle$ and $\left\langle e_{1}, e_{2}\right\rangle-\left\langle e_{1}^{\prime}, e_{2}^{\prime}\right\rangle$ vanish when $t_{1}=l_{1} / 2$, and have the same signs elsewhere. There- 
fore, the previous equation holds if and only if

$$
-\left\langle V_{1}, V_{2}\right\rangle+\left\langle V_{1}^{\prime}, V_{2}^{\prime}\right\rangle=0 \quad \text { and } \quad\left\langle e_{1}, e_{2}\right\rangle-\left\langle e_{1}^{\prime}, e_{2}^{\prime}\right\rangle=0,
$$

and this is equivalent to $t_{1}=l_{1} / 2$.

Finally, the study of the traces of $\rho \beta_{1}$ and $\rho \beta_{2}$, which are equal, gives the last condition.

The converse is analogous to that in Theorem 5.7

By looking at the explicit equations obtained in Theorem 5.7 and Propositions 5.14 and 5.15 , we immediately get the following corollary, verifying Theorem 6.4 of $[20]$ in this case.

Corollary 5.16. The closure $\overline{\mathcal{P}}_{\alpha_{1}, \alpha_{2} ; \beta_{1}, \beta_{2}}$ of $\mathcal{P}_{\alpha_{1}, \alpha_{2} ; \beta_{1}, \beta_{2}}$ in $\mathcal{Q F}$ meets $\mathcal{F}$ along the closed simplex of minima $\overline{\mathcal{S}}_{\alpha_{1}, \alpha_{2} ; \beta_{1}, \beta_{2}}$. The closure of the subpleating varieties of $\overline{\mathcal{P}}_{\alpha_{1}, \alpha_{2} ; \beta_{1}, \beta_{2}}$ computed above meets $\mathcal{F}$ along the corresponding simplices of minima.

Remark. When we reach a point at which $\operatorname{tr} \rho \beta_{1}=2$ or $\operatorname{tr} \rho \beta_{2}=2$ on an $\alpha$-line, we have arrived at a point in the boundary of $\mathcal{Q F}$. Such a point is a cusp group at which one of the curves $\beta_{1}$ or $\beta_{2}$ (or both) is pinched. Even though this point is not in $\mathcal{Q F}$, we can still use Fenchel-Nielsen $\alpha$-coordinates to write down the representation at such a point. This would be impossible using Fenchel-Nielsen $\beta$-coordinates. To reach cusp groups at which the $\alpha_{i}$ are pinched, on the other hand, one should follow $\beta$-lines (defined in the obvious way by analogy to the $\alpha$ lines). To write down the corresponding representations, one needs Fenchel-Nielsen $\beta$-coordinates, compare the remark on page 628 .

5.4. Example 2: The pleating variety $\mathcal{P}_{\alpha_{1}, \alpha_{2} ; \beta_{1}, \beta_{2}}$. In this final section we compute the pleating variety $\mathcal{P}_{\alpha_{1}, \alpha_{2} ; \beta_{1}, \delta_{1}}$ and some adjacent pleating varieties in $\mathcal{Q F}$. Consider the subset $\mathcal{W}=\left\{\rho=\left(l_{1}+i \varphi_{1}, l_{2}+i \varphi_{2}, t_{1}+i \theta_{1}, t_{2}+i \theta_{2}\right): \varphi_{1}=\right.$ $\left.\varphi_{2}=0,0<\theta_{1}<\pi, 0<\theta_{2}<\pi\right\}$. Clearly $\mathcal{P}_{\alpha_{1}, \alpha_{2} ; \beta_{1}, \delta_{1}} \subset \mathcal{W}$.

We first find the real locus of $\beta_{1}, \delta_{1}$, that is, the subset of $\mathcal{W}$ where the traces of $\rho \beta_{1}$ and $\rho \delta_{1}$ are real (Lemma 5.17). We continue by finding the purely hyperbolic locus, that is, the locus where these traces are greater than two in absolute value (Lemma 5.18). At any point $\rho$ in the purely hyperbolic locus, we have, as described on page 643 , the $\rho$-invariant pleated surface $\mathrm{Pl}_{\beta_{1}, \delta_{1}}$ consisting of planar components glued along the axes of $\rho \beta_{1}, \rho \delta_{1}$ and their images under the group. We study where the bending angles $\theta_{\beta_{1}}, \theta_{\delta_{1}}$ of $\mathrm{Pl}_{\beta_{1}, \delta_{1}}$ either vanish or are equal to $\pm \pi$, giving a curve which divides the purely hyperbolic locus into two connected regions. Each component is foliated by suitably defined ' $\alpha$-lines' emanating from Fuchsian space (Proposition 5.19). Finally, we study in which of these regions the angles $\theta_{\beta_{1}}, \theta_{\delta_{1}}$ have the same sign. This will be exactly the region in which $\mathrm{Pl}_{\beta_{1}, \delta_{1}}$ is embedded and bounds a convex half space, thus completing our description of the pleating variety $\mathcal{P}_{\alpha_{1}, \alpha_{2} ; \beta_{1}, \delta_{1}}$ (Theorem5.21).

Lemma 5.17. Let $\rho \in \mathcal{W}$. Then $\operatorname{tr} \rho \beta_{1}, \operatorname{tr} \rho \delta_{1}$ are real if and only if $t_{1}=t_{2}=0$.

Proof. By the same method as in Lemma 4.14, we find that

$$
\operatorname{tr} \rho \delta_{1}=-4 \sinh ^{2}\left(l_{2} / 2\right) \sinh ^{2} d \cosh ^{2}\left(\tau_{1} / 2\right)+2
$$

with $\tau_{1}=t_{1}+i \theta_{1}$. Thus $\operatorname{tr} \rho \delta_{1}$ is real if and only if $\cosh ^{2} \frac{\tau_{1}}{2}$ is real. Since $\theta_{1} \neq 0, \pm \pi$, we must have $t_{1}=0$. By Proposition $3.2 \operatorname{tr} \rho \beta_{1}$ is real if

$$
\cos \left(\theta_{1} / 2\right) \sin \left(\theta_{2} / 2\right)\left\langle V_{1}, e_{2}\right\rangle+\sin \left(\theta_{1} / 2\right) \cos \left(\theta_{2} / 2\right)\left\langle e_{1}, V_{2}\right\rangle=0 .
$$


We observe that, if $t_{1}=0$, then either $\left\langle V_{1}, e_{2}\right\rangle$ and $\left\langle e_{1}, V_{2}\right\rangle$ are both equal to zero, or have the same (strict) sign. On the other hand, $\cos \frac{\theta_{1}}{2} \sin \frac{\theta_{2}}{2}$ and $\sin \frac{\theta_{1}}{2} \cos \frac{\theta_{2}}{2}$ are both greater than or equal to zero and do not both vanish. Therefore, (13) holds if and only if $\left\langle V_{1}, e_{2}\right\rangle=\left\langle e_{1}, V_{2}\right\rangle=0$, which is equivalent to $t_{1}=t_{2}=0$. The converse is immediate from the formulae for $\operatorname{tr} \rho \beta_{1}, \operatorname{tr} \rho \delta_{1}$.

Denote by $\mathcal{H}_{\beta_{1}, \delta_{1}}$ the purely hyperbolic locus, which, by the previous lemma, is the subset

$$
\mathcal{H}_{\beta_{1}, \delta_{1}}=\left\{\rho \in \mathcal{W}: t_{1}=t_{2}=0,\left|\operatorname{tr} \rho \beta_{1}\right|>2,\left|\operatorname{tr} \rho \delta_{1}\right|>2\right\} .
$$

For fixed values of $l_{1}, l_{2}$, this is a subset of the plane parametrised by $\theta_{1}, \theta_{2}$. It is foliated by the curves $\sigma_{c}$ defined by $\tan \left(\theta_{2} / 2\right)=c \tan \left(\theta_{1} / 2\right)$, for $c \in \mathbb{R}^{+}$. These curves are analogous to the $\alpha$-lines we used in the previous example. (See Figure 9: $\mathcal{H}_{\beta_{1}, \delta_{1}}$ is the first quadrant and $\sigma_{c}$ the dotted lines.)

Lemma 5.18. $\mathcal{H}_{\beta_{1}, \delta_{1}}$ is connected. More precisely, for fixed $l_{1}, l_{2}$ and for each $c>0, \mathcal{H}_{\beta_{1}, \delta_{1}} \cap \sigma_{c}$ has one connected component, whose closure contains the point $\left(l_{1}, l_{2}, 0,0\right)$.

Proof. We study the functions $\operatorname{tr} \beta_{1}, \operatorname{tr} \delta_{1}$ along the curves $\sigma_{c}$. The formulae are

$$
\begin{aligned}
& \operatorname{tr} \delta_{1}=-4 \sinh ^{2}\left(l_{2} / 2\right) \sinh ^{2} d \cos ^{2}\left(\theta_{1} / 2\right)+2, \\
& \operatorname{tr} \beta_{1}=2 \cosh d \cos \left(\theta_{1} / 2\right) \cos \left(\theta_{2} / 2\right)-2 \sin \left(\theta_{1} / 2\right) \sin \left(\theta_{2} / 2\right) .
\end{aligned}
$$

From the formula, $\operatorname{tr} \delta_{1}$ is increasing as $\theta_{1}$ moves in the interval $[0, \pi]$, and the maximum value is 2 (recall that $\operatorname{tr} \delta_{1}<-2$ at $\theta_{1}=0$ ). Since $c>0, \theta_{2}$ is increasing with $\theta_{1}$; then $\operatorname{tr} \beta_{1}$ is decreasing in the same interval, and the minimum value is -2 . Thus, the result follows.

Proposition 5.19. Let $\rho \in \mathcal{H}_{\beta_{1}, \delta_{1}}$, and let $\theta_{\beta_{1}}, \theta_{\delta_{1}}$ be the bending angles of $\mathrm{Pl}_{\beta_{1}, \delta_{1}}$. Then:

(a) $\theta_{\delta_{1}}=0$ if and only if $\tan \left(\theta_{2} / 2\right)=\frac{\sinh \left(l_{1} / 2\right)}{\sinh \left(l_{2} / 2\right)} \tan \left(\theta_{1} / 2\right) ; \theta_{\delta_{1}} \neq \pm \pi$,

(b) $\theta_{\beta_{1}} \neq 0, \pm \pi$

Proof. (a) Since $\left|\operatorname{tr} \rho \delta_{1}\right|>2$, if the angle $\theta_{\delta_{1}}$ is 0 or $\pm \pi$, then the two flat pieces meeting along the axis of $\rho \delta_{1}$ are coplanar, so that $\mathrm{Pl}_{\beta_{1}, \delta_{1}}$ is only bent along the axis of $\rho \beta_{1}$ and its images under the group. Then the group $\Gamma_{\beta_{1}}=\rho\left(\pi_{1}\left(S-\beta_{1}\right)\right)$ obtained by cutting the surface along $\beta_{1}$ leaves a plane invariant. In particular, $\rho \beta_{2}$ leaves this plane invariant, since the curve $\beta_{2}$ is contained in $S-\beta_{1}$, and therefore its trace is either real or purely imaginary (in the latter case, $\rho \beta_{2}$ is loxodromic with rotation angle equal to $\pi$ ).

By Proposition 3.2 the condition for the trace to be purely imaginary is

$$
\cos \left(\theta_{1} / 2\right) \cos \left(\theta_{2} / 2\right)\left\langle V_{1}^{\prime}, V_{2}^{\prime}\right\rangle-\sin \left(\theta_{1} / 2\right) \sin \left(\theta_{2} / 2\right)\left\langle e_{1}^{\prime}, e_{2}^{\prime}\right\rangle=0 .
$$

Since $t_{1}=t_{2}=0$, the four points $V_{i}, V_{i}^{\prime}$ are the four finite vertices of one of the hexagons shown in Figure 2 with alternate sides $l_{1} / 2, l_{2} / 2,0$. In particular, $\left\langle e_{1}^{\prime}, e_{2}^{\prime}\right\rangle=1$, while $\left\langle V_{1}^{\prime}, V_{2}^{\prime}\right\rangle$ is always strictly negative, and therefore the above equation does not have any solution for $\theta_{1} \in(0, \pi), \theta_{2} \in(0, \pi)$. Thus $\operatorname{tr} \rho \beta_{2}$ must be real and, by Proposition [3.2, we have

$$
\tan \frac{\theta_{2}}{2}=-\frac{\left\langle e_{1}^{\prime}, V_{2}^{\prime}\right\rangle}{\left\langle V_{1}^{\prime}, e_{2}^{\prime}\right\rangle} \tan \frac{\theta_{1}}{2}=\frac{\sinh \frac{l_{1}}{2}}{\sinh \frac{l_{2}}{2}} \tan \frac{\theta_{1}}{2} .
$$


(The last equality derives from the trigonometric relations for $\left\langle V_{1}^{\prime}, e_{2}^{\prime}\right\rangle=-\sinh g_{1}$ and $\left\langle e_{1}^{\prime}, V_{2}^{\prime}\right\rangle=\sinh g_{2}$ in terms of $l_{1}, l_{2}$, where $g_{i}$ is the distance in the hexagon from $V_{i}^{\prime}$ to its opposite infinite side.) Thus, if $\theta_{\delta_{1}}=0$ or $\pm \pi, \rho$ satisfies the equation in the statement.

On the other hand, if $\rho$ satisfies this equation, $\operatorname{tr} \rho \beta_{2}$ is real, so that the following elements of $\Gamma_{\beta_{1}}$ have real trace: $\rho \beta_{i}, \rho \delta_{i}, \rho \omega_{i}$ for $i=1,2$, where $\omega_{1}, \omega_{2}$ are loops in $S-\beta_{1}$ around the punctures (the formula for $\operatorname{tr} \rho \delta_{2}$ is obtained in the same way as for $\delta_{1}$, and is real because $t_{2}=0$ ). Then, by Lemma 5.20 below, $\Gamma_{\beta_{1}}$ is Fuchsian and hence $\theta_{\delta_{1}}=0$.

(b) As before, if $\theta_{\beta_{1}}=0, \pm \pi$ and $\left|\operatorname{tr} \rho \beta_{1}\right|>2$, the two flat pieces of $\mathrm{Pl}_{\beta_{1}, \delta_{1}}$ meeting along the axis of $\rho \beta_{1}$ are coplanar. Consider the group $\Gamma_{\delta_{1}}=\rho\left(\pi_{1}\left(S_{1}\right)\right)$, where $S_{1}$ is the component of genus one of $S-\delta_{1}$. This group is generated by $\rho \alpha_{2}, \rho \beta_{1}$. If $\theta_{\beta_{1}}=0$, then $\Gamma_{\delta_{1}}$ is Fuchsian, and $\operatorname{tr} \rho\left(\beta_{1} \alpha_{2}\right)$ is real. From Theorem 3.1 we get

$$
\operatorname{tr} \rho\left(\beta_{1} \alpha_{2}\right)=\operatorname{tr}\left(T_{L_{1}}\left(i \theta_{1}\right) T_{M}(d) T_{L_{1}}\left(l_{2}+i \theta_{2}\right) T_{M}(d)\right),
$$

and from Proposition 3.2 this trace is real if and only if

$$
\cos \left(\theta_{1} / 2\right) \sin \left(\theta_{2} / 2\right)\left\langle V_{1}(0), e_{2}\left(l_{2}\right)\right\rangle+\sin \left(\theta_{1} / 2\right) \cos \left(\theta_{2} / 2\right)\left\langle e_{1}(0), V_{2}\left(l_{2}\right)\right\rangle=0 .
$$

Now $\left\langle e_{1}(0), V_{2}\left(l_{2}\right)\right\rangle=-\sinh \left(l_{2} / 2\right)$ and $\left\langle V_{1}(0), e_{2}\left(l_{2}\right)\right\rangle=-\sinh h_{1}$, where $h_{1}$ is the distance from $V_{1}$ to the opposite side. So the previous equation does not have any solution with $\theta_{1} \in(0, \pi), \theta_{2} \in(0, \pi)$, and we get a contradiction.

It is left to prove that $\theta_{\beta_{1}}$ cannot be equal to $\pm \pi$. To see it, we first look at the pleated surface $\mathrm{Pl}_{\beta_{1}, \delta_{1}}$ when the angle $\theta_{\beta_{1}}$ is smaller than $\pi$. Let $F_{1}, F_{2}$ be the two flat pieces meeting at the axis of $\rho \beta_{1}$. One of these flat pieces, for instance $F_{1}$, also contains the axis of $\rho\left(\alpha_{2}^{-1} \beta_{1} \alpha_{2}\right)$ and the other contains the axis of $\rho\left(\alpha_{2} \beta_{1} \alpha_{2}^{-1}\right)$. Let us denote these three axes by $L, L^{-}, L^{+}$, respectively. The flat pieces have the "same" orientation on $\mathrm{Pl}_{\beta_{1}, \delta_{1}}$, meaning that, assuming $F_{1}$ is to the right of $L^{-}$, then $F_{1}$ is to the left of $L$, and $F_{2}$ is to the right of $L$ and to the left of $L^{+}$. Now the element $\rho \alpha_{2}$, which is purely hyperbolic, maps $L^{-}$onto $L$, and the flat piece of $\mathrm{Pl}_{\beta_{1}, \delta_{1}}$ to its right (that is, $F_{1}$ ) onto the flat piece to the right of $L$ (that is, $F_{2}$ ).

If $\theta_{\beta_{1}}= \pm \pi$, then $\mathrm{Pl}_{\beta_{1}, \delta_{1}}$ is folded along $L$, so that $F_{1}$ and $F_{2}$ are superposed. Hence, in this case $\rho \alpha_{2}$ leaves invariant the plane $P$ containing $L^{-}$and $L$, and maps the halfplane to the right of $L^{-}$to the halfplane to the left of $L$. But this implies that $\rho \alpha_{2}$ reverses orientation on $P$, which is impossible because it is purely hyperbolic.

We now prove Lemma 5.20 used in the previous proposition, which is based on the following result by Horowitz [8]: if a group $G \subset P S L(2, \mathbb{C})$ is generated by $A, B, C$ and $\operatorname{tr} A, \operatorname{tr} B, \operatorname{tr} C, \operatorname{tr} A B, \operatorname{tr} A C, \operatorname{tr} B C, \operatorname{tr} A B C$ are real, then the trace of any element of $G$ is real (and hence $G$ is Fuchsian).

Lemma 5.20. Let $\Gamma_{\beta_{1}}$ be the group defined in the proof of Proposition 5.19(b), and suppose that the traces of $\rho \beta_{i}, \rho \delta_{1}, \rho \omega_{i}$ are real, for $i=1,2$. Then $\Gamma_{\beta_{1}}$ is Fuchsian.

Proof. With the notation for the fundamental group of $S$ given in Section 3, the group $\Gamma_{\beta_{1}}$ is generated by $A=\rho \beta_{1}, B=\rho \omega_{1}$, and $C=\rho\left(\alpha_{1} \beta_{1} \alpha_{1}^{-1}\right)$, with $\omega_{1}=$ $\alpha_{1} \alpha_{2}^{-1}$ a loop around a puncture. Using the fact that $\operatorname{tr} \rho \beta_{i}, \operatorname{tr} \rho \delta_{i}, \operatorname{tr} \rho \omega_{i}$ are real and the usual trace relations, one can check that the traces of $A B, A C, B C$ and $A B C$ are all real. By Horowitz' result, $\Gamma_{\beta_{1}}$ is Fuchsian. 
Remark. Note that in our first example, $\mathcal{P}_{\alpha_{1}, \alpha_{2} ; \beta_{1}, \beta_{2}}$, the question about the bending angles $\theta_{\beta_{i}}$ being 0 or $\pm \pi$ was sorted out more quickly because of the symmetry between $\left\{\alpha_{1}, \alpha_{2}\right\}$ and $\left\{\beta_{1}, \beta_{2}\right\}$ (see Proposition 5.9).

We finally obtain the complete description of $\mathcal{P}_{\alpha_{1}, \alpha_{2} ; \beta_{1}, \delta_{1}}$.

Theorem 5.21. The pleating variety $\mathcal{P}_{\alpha_{1}, \alpha_{2} ; \beta_{1}, \delta_{1}}$ is the subset of points $\left(l_{1}+i \varphi_{1}\right.$, $\left.l_{2}+i \varphi_{2}, t_{1}+i \theta_{1}, t_{2}+i \theta_{2}\right)$ satisfying

$$
\begin{array}{r}
\varphi_{1}=\varphi_{2}=0, \\
0<\theta_{1}, \theta_{2}<\pi, \\
t_{1}=t_{2}=0, \\
\tan \left(\theta_{2} / 2\right)<\sinh \left(l_{1} / 2\right)\left(\sinh \left(l_{2} / 2\right)\right)^{-1} \tan \left(\theta_{1} / 2\right), \\
\cosh d \cos \left(\theta_{1} / 2\right) \cos \left(\theta_{2} / 2\right)-\sin \left(\theta_{1} / 2\right) \sin \left(\theta_{2} / 2\right)>1, \\
2 \sinh ^{2}\left(l_{2} / 2\right) \sinh ^{2} d \cos ^{2}\left(\theta_{1} / 2\right)>1 .
\end{array}
$$

Proof. In view of Lemmas [5.17, [5.18 and Proposition [5.19] we only need to study the condition that the angles $\theta_{\beta_{1}}, \theta_{\delta_{1}}$ have the same sign. The curve $\sigma_{c_{0}}$ with $c_{0}=\sinh \left(l_{1} / 2\right) / \sinh \left(l_{2} / 2\right)$ divides the hyperbolic locus $\mathcal{H}_{\beta_{1}, \delta_{1}}$ into two connected components; by Proposition [5.19, in each component the signs of $\theta_{\beta_{1}}$ and $\theta_{\delta_{1}}$ are constant. We can find these signs either by direct computation at some point in each region, or by appealing to Lemma 5.2 To do this, take a curve $\sigma_{c}$ with $c>0$ and $c \neq c_{0}$. Along $\sigma_{c}$ the traces of $\rho \alpha_{1}, \rho \alpha_{2}, \rho \beta_{1}, \rho \delta_{1}$ are real and, as we approach $\mathcal{F}$, the limiting ratio $\theta_{\alpha_{2}} / \theta_{\alpha_{1}}$ is $c$. By the lemma, the limits $b_{1}=\lim \left(\theta_{\beta_{1}} /\left(\left|\theta_{\beta_{1}}\right|+\left|\theta_{\delta_{1}}\right|\right)\right)$, $d_{1}=\lim \left(\theta_{\delta_{1}} /\left(\left|\theta_{\beta_{1}}\right|+\left|\theta_{\delta_{1}}\right|\right)\right)$ and $k=\lim \left(\left(\left|\theta_{\beta_{1}}\right|+\left|\theta_{\delta_{1}}\right|\right) /\left(\left|\theta_{\alpha_{1}}\right|+\left|\theta_{\alpha_{2}}\right|\right)\right)$ exist and, at $p=\sigma_{c} \cap \mathcal{F}$ :

$$
\left.\left(\frac{1}{1+c}\right) \frac{\partial}{\partial t_{\alpha_{1}}}\right|_{p}+\left.\left(\frac{c}{1+c}\right) \frac{\partial}{\partial t_{\alpha_{2}}}\right|_{p}=k\left(\left.b_{1} \frac{\partial}{\partial t_{\beta_{1}}}\right|_{p}+\left.d_{1} \frac{\partial}{\partial t_{\delta_{1}}}\right|_{p}\right) .
$$

Now the plane in Figure 6 is the linear span of the tangent vectors $\left.\frac{\partial}{\partial t_{\alpha_{1}}}\right|_{p},\left.\frac{\partial}{\partial t_{\alpha_{2}}}\right|_{p}$ (which is the same as the span of the vectors $\left.\frac{\partial}{\partial t_{\beta_{1}}}\right|_{p},\left.\frac{\partial}{\partial t_{\delta_{1}}}\right|_{p}$ ). From this figure we see that, if $c$ is large, then the positive combination $\left.\frac{1}{1+c} \frac{\partial}{\partial t_{\alpha_{1}}}\right|_{p}+\left.\frac{c}{1+c} \frac{\partial}{\partial t_{\alpha_{2}}}\right|_{p}$ is close to $\left.\frac{\partial}{\partial t_{\alpha_{2}}}\right|_{p}$ and therefore is equal to a linear combination of $\left.\frac{\partial}{\partial t_{\beta_{1}}}\right|_{p},\left.\frac{\partial}{\partial t_{\delta_{1}}}\right|_{p}$ with coefficients of different signs. On the other hand, if $c$ is small, then $\left.\frac{1}{1+c} \frac{\partial}{\partial t_{\alpha_{1}}}\right|_{p}+\left.\frac{c}{1+c} \frac{\partial}{\partial t_{\alpha_{2}}}\right|_{p}$ is close in direction to $\left.\frac{\partial}{\partial t_{\alpha_{1}}}\right|_{p}$ and is opposite to a positive combination of $\left.\frac{\partial}{\partial t_{\beta_{1}}}\right|_{p},\left.\frac{\partial}{\partial t_{\delta_{1}}}\right|_{p}$. Therefore, $\theta_{\beta_{1}}, \theta_{\delta_{1}}$ have the same sign in the region foliated by $\sigma_{c}$ with $c<c_{0}$; thus we have (17).

Neighbouring pleating varieties. Similar methods may be used to study other pleating varieties which also meet $\mathcal{F}$ in the plane $\Pi=\left\{t_{1}=t_{2}=0\right\}$. Let $\Pi_{\mathbb{C}}$ be the set of points $\left(l_{1}+i \varphi_{1}, l_{2}+i \varphi_{2}, t_{1}+i \theta_{1}, t_{2}+i \theta_{2}\right) \in \mathcal{R}$ satisfying $\varphi_{1}=\varphi_{2}=$ $0, \theta_{1}, \theta_{2} \in(-\pi, \pi)$ and $t_{1}=t_{2}=0$. Thus $\Pi_{\mathbb{C}}$ extends $\Pi$ into $\mathcal{Q F}$. If $\rho \in \Pi_{\mathbb{C}}$, then $\rho \alpha_{1}, \rho \alpha_{2}, \rho \beta_{1}, \rho \delta_{1}, \rho \delta_{2}$ all have real trace (the formula for $\operatorname{tr} \rho \delta_{2}$ is analogous to that for $\left.\operatorname{tr} \rho \delta_{1}\right)$. Since $\left\{\alpha_{1}, \alpha_{2}\right\},\left\{\alpha_{2}, \delta_{1}\right\},\left\{\alpha_{1}, \delta_{2}\right\},\left\{\beta_{1}, \delta_{1}\right\},\left\{\beta_{1}, \delta_{2}\right\}$ are pants decompositions of $S$, we have the following $\rho$-invariant pleated surfaces, whenever $\rho$ is in the purely hyperbolic locus of the corresponding curves:

$$
\mathrm{Pl}_{\alpha_{1}, \alpha_{2}}, \mathrm{Pl}_{\alpha_{2}, \delta_{1}}, \mathrm{Pl}_{\alpha_{1}, \delta_{2}}, \mathrm{Pl}_{\beta_{1}, \delta_{1}}, \mathrm{Pl}_{\beta_{1}, \delta_{2}} \text {. }
$$




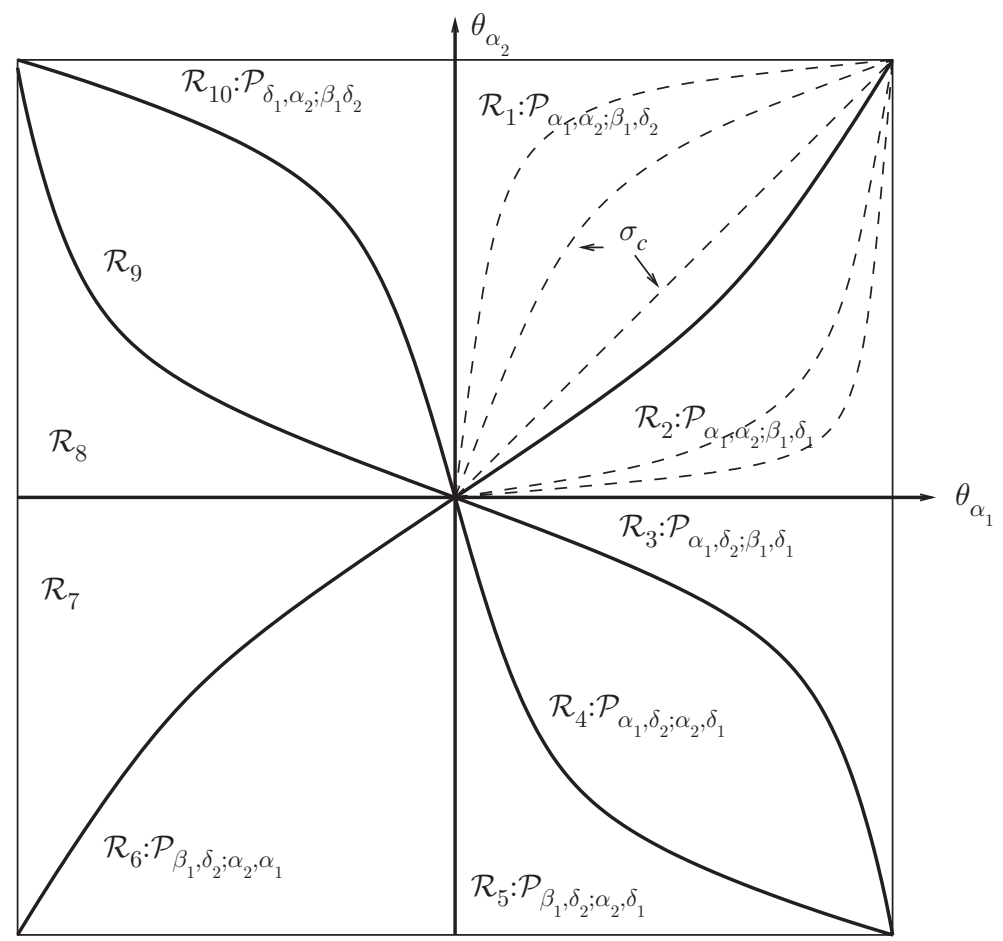

Figure 9. Pleating varieties meeting at $\rho \in \Pi$. The picture shows the intersection of $\Pi_{\mathbb{C}}$ with the locus of a fixed pair of values for $l_{1}, l_{2}$.

A necessary condition for the pleating variety $\mathcal{P}_{\mathcal{A}, \mathcal{B}}$ to be non-empty is that the pants decompositions $\mathcal{A}$ and $\mathcal{B}$ fill up the surface. Thus, using the above pairs of curves, we can study the pleating varieties

$$
\mathcal{P}_{\alpha_{1}, \alpha_{2} ; \beta_{1}, \delta_{2}}, \mathcal{P}_{\alpha_{1}, \alpha_{2} ; \beta_{1}, \delta_{1}}, \mathcal{P}_{\alpha_{1}, \delta_{2} ; \beta_{1}, \delta_{1}}, \mathcal{P}_{\alpha_{1}, \delta_{2} ; \alpha_{2}, \delta_{1}}, \mathcal{P}_{\beta_{1}, \delta_{2} ; \alpha_{2}, \delta_{1}},
$$

together with their common boundaries $\mathcal{P}_{\alpha_{1}, \alpha_{2} ; \beta_{1}}, \mathcal{P}_{\alpha_{1} ; \beta_{1}, \delta_{1}}, \mathcal{P}_{\alpha_{1}, \delta_{2} ; \delta_{1}}, \mathcal{P}_{\delta_{2} ; \alpha_{2}, \delta_{1}}$, and so on. Moreover, interchanging the bending lines on the two sides, we also have $\mathcal{P}_{\beta_{1}, \delta_{2} ; \alpha_{1}, \alpha_{2}}$ and so on. The plane $\Pi_{\mathbb{C}} \cap \mathcal{Q F}$ is exactly the union of all these pleating varieties, as illustrated in Figure 9.

To verify this picture, we begin by studying the common boundary between two of the above pleating varieties, obtained when suitable bending angles vanish. For example, the common boundary of $\mathcal{P}_{\alpha_{1}, \alpha_{2} ; \beta_{1}, \delta_{2}}$ and $\mathcal{P}_{\alpha_{1}, \alpha_{2} ; \beta_{1}, \delta_{1}}$ is $\mathcal{P}_{\alpha_{1}, \alpha_{2} ; \beta_{1}}$, obtained when the bending angle $\theta_{\delta_{2}}$ of $\mathrm{Pl}_{\beta_{1}, \delta_{2}}$ is equal to zero, or equivalently when the bending angle $\theta_{\delta_{1}}$ of $\mathrm{Pl}_{\beta_{1}, \delta_{1}}$ is zero. By Proposition [5.19, this condition gives the curve $\sigma_{c_{0}}$ defined by $\tan \left(\theta_{2} / 2\right)=c_{0} \tan \left(\theta_{1} / 2\right)$, with $c_{0}=\sinh \left(l_{1} / 2\right)\left(\sinh \left(l_{2} / 2\right)\right)^{-1}$. Proceeding in the same way as in Proposition 5.19, we obtain that $\mathcal{P}_{\alpha_{1}, \delta_{2} ; \delta_{1}} \cap \Pi_{\mathbb{C}}$ is contained in the curve $\sigma_{c_{1}}$, with $c_{1}=-\cosh d$, and $\mathcal{P}_{\delta_{2} ; \alpha_{2}, \delta_{1}} \cap \Pi_{\mathbb{C}}$ is contained in the curve $\sigma_{c_{2}}$, with $c_{2}=-(\cosh d)^{-1}$.

The curves $\sigma_{c_{0}}, \sigma_{c_{1}}, \sigma_{c_{2}}$ and the two coordinate axes $\theta_{1}=0, \theta_{2}=0$ partition $\Pi_{\mathbb{C}}$ into ten connected regions, each containing the intersection of $\Pi_{\mathbb{C}}$ with one of the above pleating varieties. Each pleating variety is itself a connected region whose 
outer boundary (not shown in the figure) consists entirely of cusp groups in which at least one of the bending lines $\beta_{1}, \delta_{1}$ or $\delta_{2}$ is parabolic. We have already verified this for $\mathcal{P}_{\alpha_{1}, \alpha_{2} ; \beta_{1}, \delta_{1}}$, and the details for the rest can be worked out in a similar way. The details for $\mathcal{P}_{\alpha_{1}, \alpha_{2} ; \beta_{1}, \delta_{2}}$ are almost identical, but the others require some more work which we leave to the untiring reader to explore 2

Non-singularity.

Theorem 5.22. The length functions $\left(\lambda_{\alpha_{1}}(q), \lambda_{\alpha_{2}}(q), \lambda_{\beta_{1}}(q), \lambda_{\delta_{1}}(q)\right)$ are local holomorphic coordinates for $\mathcal{Q} \mathcal{F}$ in a neighbourhood of $\mathcal{P}_{\alpha_{1}, \alpha_{2} ; \beta_{1}, \delta_{1}}$.

Proof. We have to prove that the map

$$
\Psi: q \mapsto\left(\lambda_{\alpha_{1}}(q), \lambda_{\alpha_{2}}(q), \lambda_{\beta_{1}}(q), \lambda_{\delta_{1}}(q)\right)
$$

is invertible in a neighbourhood of each point in $\mathcal{P}_{\alpha_{1}, \alpha_{2} ; \beta_{1}, \delta_{1}}$. Since $i\left(\alpha_{2}, \delta_{1}\right)=0$, the bottom right entry in the Jacobian matrix of $\Psi$ vanishes, and we therefore have only to show that neither $\frac{\partial \lambda_{\beta_{1}}}{\partial \tau_{\alpha_{2}}}$ nor $\frac{\partial \lambda_{\delta_{1}}}{\partial \tau_{\alpha_{1}}}$ vanishes. The first of these two expressions is non-zero as in the case of $\mathcal{P}_{\alpha_{1}, \alpha_{2} ; \beta_{1}, \beta_{2}}$ (since $i\left(\alpha_{2}, \beta_{1}\right) \neq 0$ ), and the second is easily verified using the explicit formula for $\operatorname{tr} \delta_{1}$ in Lemma 5.17 .

\section{ACKNOWLEDGMENTS}

Most of this work was done during various visits of the first author to the University of Warwick with grants from TMR Programme, Nr. ERB4001GT973983, and SEUID-Royal Society, RS1997 0051376235. Partial support for the first author has been also provided by DGICYT grants PB96-0659 and BFM2000-0621. The second author is grateful for the support of her EPSRC Senior Research Fellowship. We thank Yohei Komori for his careful reading of this paper.

\section{REFERENCES}

[1] F. Bonahon and J-P. Otal. Laminations mesurées de plissage des variétés hyperboliques de dimension 3, preprint, 2001.

[2] R. D. Canary, D. B. A. Epstein and P. Green. Notes on notes of Thurston. In D. B. A. Epstein, editor, "Analytical and Geometric Aspects of Hyperbolic Space", LMS Lecture Notes 111, 3-92. Cambridge University Press, 1987. MR 89e:57008

[3] R. Díaz and C. Series. Limits of lines of minima in Thurston's boundary of Teichmüller space, Algebraic and Geometric Topology 3, 207-234, 2003.

[4] D. B. A. Epstein and A. Marden. Convex hulls in hyperbolic space, a theorem of Sullivan, and measured pleated surfaces. In D. B. A. Epstein, editor, "Analytical and Geometric Aspects of Hyperbolic Space", LMS Lecture Notes 111, 112-253. Cambridge University Press, 1987. MR 89c:52014

[5] A. Fahti, P. Laudenbach, and V. Poénaru. Travaux de Thurston sur les surfaces, Astérisque 66-67. Société Mathématique de France, 1979. MR 82m:57003

[6] F. Gardiner and L. Keen. Holomorphic motions and quasi-Fuchsian manifolds, Contemp. Math. 240, 159-173, 1999. MR 2000k:30023

[7] P.A. Griffiths and J. Harris. Principles of Algebraic geometry. Wiley, 1978. MR 80b:14001

[8] R.D. Horowitz. Characters of free groups represented in the two dimensional special linear group, Comm. Pure Appl. Math. 25, 635-649, 1972. MR 47:3542

[9] L. Keen and C. Series. Pleating coordinates for the Maskit embedding of the Teichmüller space of punctured tori, Topology 32, 719-749, 1993. MR 95g:32030

\footnotetext{
${ }^{2}$ For example, in the case of $\mathcal{P}_{\alpha_{1}, \delta_{2} ; \beta_{1}, \delta_{1}}$, we have assumed from the outset that the $\operatorname{tr} \alpha_{2} \in \mathbb{R}$, without proving that this follows automatically from the condition that the other four traces are real. Thus our method has located a region of $\Pi_{\mathbb{C}}$ contained in $\mathcal{P}_{\alpha_{1}, \delta_{2} ; \beta_{1}, \delta_{1}}$, but has not demonstrated that this region is the entire pleating variety.
} 
[10] L. Keen and C. Series. Continuity of convex hull boundaries, Pacific J. Math. 168(1), 183-206, 1995. MR 96d:30055

[11] L. Keen and C. Series. How to bend pairs of punctured tori. In J. Dodziuk and L. Keen, editors, "Lipa's Legacy", Contemp. Math. 211, 359-388, 1997. MR 98m:30063

[12] L. Keen and C. Series. Pleating invariants for punctured torus groups, Topology, 2003.

[13] L. Keen and C. Series. The Riley slice of Schottky space, Proceedings of the London Mathematical Society 3, 72-90, 1994. MR 95j:32033

[14] S. Kerckhoff. The Nielsen realization problem, Ann. of Math. 117, 235-265, 1983. MR 85e:32029

[15] S. Kerckhoff. Lines of Minima in Teichmüller space, Duke Math J. 65, 187-213, 1992. MR 93b:32027

[16] Y. Komori and C. Series. Pleating coordinates for the Earle embedding, Ann. de la Fac. des Sciences de Toulouse, Vol. X, 69-105, 2001.

[17] C. Kourouniotis, Complex length coordinates for quasi-Fuchsian groups, Mathematika 41(1), 173-188, 1994. MR 96g:30079

[18] I. Kra. On lifting Kleinian groups to $S L(2, \mathbb{C})$. In: "Differential Geometry and Complex Analysis", I. Chavel and H. Farkas, editors, 181-193. Springer-Verlag, 1985. MR 86h:30078

[19] C. Series. Lectures on pleating coordinates for once punctured tori, In Hyperbolic Spaces and Related topics, RIMS Kokyuroku 1104, Kyoto, 30-108, 1999. MR 2000m:57019

[20] C. Series. On Kerckhoff Minima and Pleating Loci for quasi-Fuchsian Groups, Geometriae Dedicata 88, 211-237, 2001. MR 2002j:30066

[21] C. Series, Limits of quasifuchsian groups with small bending, preprint 2002. arXiv:mathGT/0209190

[22] S. P. Tan, Complex Fenchel-Nielsen coordinates for quasi-Fuchsian structures, International J. Math. 5(2), 239-251, 1994. MR 94m:32030

[23] W. Thurston. Earthquakes in two-dimensional hyperbolic geometry. In D. B. A. Epstein, editor, "Low-dimensional Topology and Kleinian Groups", LMS Lecture Notes 112, 91-112. Cambridge University Press, 1987. MR 88m:57015

[24] W. Thurston. Three-dimensional Geometry and Topology, Vol.1. Princeton U.P., 1997. MR 97m:57016

Departamento de Geometría y Topología, Facultad Matemáticas, Universidad ComPlutense, 28040 Madrid, Spain

E-mail address: radiaz@mat.ucm.es

Mathematics Institute, University of Warwick, Coventry CV4 7AL, United Kingdom

E-mail address: cms@maths.warwick.ac.uk 